\title{
Molekulare Orientierung als Kontrastmechanismus in der Fluoreszenzmikroskopie und konfokale Multidetektor-Scanning-Mikroskopie
}

\author{
Dissertation \\ zur Erlangung des mathematisch-naturwissenschaftlichen Doktorgrades \\ "Doctor rerum naturalium" \\ der Georg-August-Universität zu Göttingen \\ im Promotionsprogramm Chemie \\ der Georg-August University School of Science (GAUSS)
}

Vorgelegt von

Matthias Grunwald

aus Stemwede

Göttingen 2015 
Betreuungsausschuss

Prof. Dr. Claudia Steinem, Abt. Biomolekulare Chemie, Uni Göttingen

Prof. Dr. Peter Jomo Walla, Abt. Biophysikalische Chemie, TU Braunschweig

Mitglieder der Prüfungskommission

Referent/in: $\quad$ Prof. Dr. Claudia Steinem, Abt. Biomolekulare Chemie, Uni Göttingen

Korreferent/in: Prof. Dr. Peter Jomo Walla, Abt. Biophysikalische Chemie, TU Braunschweig

Weitere Mitglieder der Prüfungskommission:

Prof. Dr. Andreas Janshoff, Abt. Biophysikalische Chemie, Uni Göttingen

Prof. Dr. Dirk Schwarzer, Abt. Dynamik an Oberflächen, MPI für biophysikalische Chemie

Dr. Sebastian Kruss, Abt. Nanobiotechnologie, Uni Göttingen

Dr. Tim Schäfer, Abt. Dynamik an Oberflächen, Uni Göttingen

Tag der mündlichen Prüfung: 24.09.2015 




\section{Inhaltsverzeichnis}

Inhaltsverzeichnis $\quad$ i

Zusammenfassung $\quad$ v

Summary vii

$\begin{array}{ll}\text { Abkürzungsverzeichnis } & \text { ix }\end{array}$

$\begin{array}{ll}\text { Vorwort } & 1\end{array}$

Molekulare Orientierung als Kontrastmechanismus in der Fluoreszenzmikroskopie 3

1.1 Einleitung . . . . . . . . . . . . . . . . . . . . 3

1.2 Theoretische Grundlagen _ . . . . . . . . . . . . . . . 6

1.2.1 Absorption und das Übergangsdipolmoment . . . . . . . . . . . . . . 6

1.2.2 Relaxation elektronisch angeregter Zustände . . . . . . . . . . 8

1.2.3 Weitfeld-Fluoreszenzmikroskopie . . . . . . . . . . . . . . . . 9 9

1.2.4 Abbildungseigenschaften eines Weitfeld-Fluoreszenzmikroskops . . . . 10

1.3 Experimentelles . . . . . . . . . . . . . . . . . . . . . . . . . . . 14

1.3.1 Experimenteller Aufbau . . . . . . . . . . . . . . . . . . . . . . . 14

1.3.1.1 Synchronisation von Modulation und Detektion . . . . . . 15

1.3.1.2 Erweiterung durch ExPAN . . . . . . . . . . . . 16

1.3.2 Proben . . . . . . . . . . . . . . . . . . . . 17

1.3.2.1 Präparation von Einzelmolekülproben . . . . . . . . . . 17

1.3.2.2 Präparation von oberflächenmarkierten Microspheres . . . . 17

1.3.2.3 Fixierte Hippocampus-Neuronen . . . . . . . . . . . . . 18

1.3.3 Algorithmus zur Auswertung von Einzelmoleküldaten . . . . . . . . . 18

1.3.4 Simulationen . . . . . . . . . . . . . . . . . . . 22

1.4 Theoretische Beschreibung von ExPAN . . . . . . . . . . . . . . . . . . 24

1.4.0.1 Fluoreszenzanisotropie . . . . . . . . . . . . . . . 27 
1.5 Ergebnisse und Diskussion . . . . . . . . . . . . . . . . . 29

1.5.1 Messungen von Einzelmolekülen . . . . . . . . . . . . . . . 29

1.5.2 Messungen von Einzelmolekülen mit ExPAN . . . . . . . . . . . 33

1.5.3 Auswertung mit dem SPEED-Algorithmus . . . . . . . . . . . . 35

1.5.3.1 Auswertung mit ExPAN . . . . . . . . . . . . . 36

1.5.4 Simulationen von Einzelmoleküldaten . . . . . . . . . . . . 37

1.5.5 Simulationen von Einzelmoleküldaten mit ExPAN . . . . . . . . 40

1.5.6 Proben mit höherer Farbstoffdichte . . . . . . . . . . . . . . . . 42

1.5.6.1 Microspheres ................... 42

1.5.6.2 Mikrotubuli in fixierten Hippocampus-Neuronen . . . . . . . 43

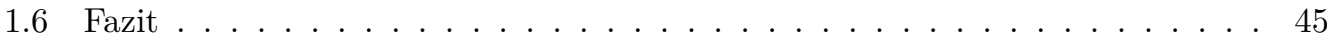

Konfokale Multidetektor-Scanning-Mikroskopie $\quad 47$

2.1 Einleitung ......................... 47

2.2 Theoretische Grundlagen _. . . . . . . . . . . . . . . . . . 50

2.2.1 Konfokale Laser-Scanning-Mikroskopie . . . . . . . . . . . . . . 50

2.2.1.1 Abbildungseigenschaften eines CLSM . . . . . . . . . . 51

2.2 .2 Image-Scanning-Mikroskopie . . . . . . . . . . . . . . . . 53

2.2.2.1 Filterung und Dekonvolution von ISM-Daten . . . . . . . . . 55

2.2.3 Zeitkorrelierte Einzelphotonenzählung . . . . . . . . . . . 57

2.2.4 Fluoreszenzlebensdauer . . . . . . . . . . . . . . . . 58

2.3 Experimentelles . . . . . . . . . . . . . . . . . 59

2.3.1 Experimenteller Aufbau . . . . . . . . . . . . . . . 59

2.3.2 Faserbündel und Detektoren . . . . . . . . . . . . . . 60

2.3.3 Elektronik und Messtechnik . . . . . . . . . . . . . . 61

2.3.4 Datenerfassungssoftware ....................... 62

2.3.5 Registrierung von MDS-Rohdaten .............. 63

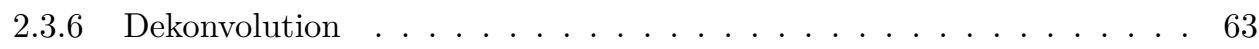

2.3.7 Fluoreszenzlebensdauer-Mikroskopie ................ 64

2.3.8 Justage des Faserbündels . . . . . . . . . . . . . . . . . . 64

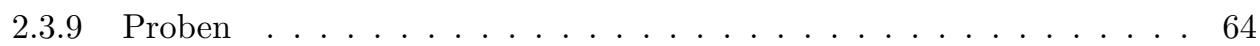

2.3.9.1 Microspheres .................... 64

2.3.9.2 Fixierte BPAE-Zellen ................ 64

2.4 Ergebnisse und Diskussion . . . . . . . . . . . . . . . . . 65

2.4.1 Theoretische Auflösung . . . . . . . . . . . . . 65

2.4.2 Reale Punktspreizfunktion . . . . . . . . . . . . . 67

2.4.3 Biologische Proben . . . . . . . . . . . . . . . 69 
2.4.4 Fluoreszenzlebensdauer-Mikroskopie . . . . . . . . . . . . . . 71

2.5 Fazit und Ausblick . . . . . . . . . . . . . . . . . . 74

$\begin{array}{lr}\text { A Anhang } & \mathbf{7 5}\end{array}$

A.1 Datenerfassungssoftware (Auszug) $\ldots \ldots \ldots \ldots$. . . . . . . . . 75

A.2 Registrierung von MDS-Daten . . . . . . . . . . . . . . . . 79

A.3 PSF Extraktion . . . . . . . . . . . . . . . . . . . . 81

A.4 Dekonvolution . . . . . . . . . . . . . . . . . . . . . . . . . . 84

$\begin{array}{lr}\text { Literaturverzeichnis } & 87\end{array}$ 



\section{Zusammenfassung}

Die vorliegende Arbeit befasst sich mit zwei neuen methodischen Ansätzen auf dem Gebiet der Fluoreszenzmikroskopie.

Im ersten Teil der Arbeit wir eine Methode vorgestellt, mit der die Winkelselektivität der Fluoreszenzanregung verbessert werden kann. Die ExPAN (excitation polarization angle narrowing) genannte Technik nutzt stimulierte Emission, um den Effekt der Photoselektion zu vergrößern. ExPAN lässt sich potentiell für verschiedene Methoden einsetzen, in denen fluoreszenzmarkierte Proben untersucht werden und ist insbesondere im Kontext von Fluoreszenzanisotropie-Messungen oder der Bestimmung von molekularen Orientierungen von Interesse. Solche Methoden finden in den Biowissenschaften breite Anwendung und werden z.B. zum Studium von Rezeptor-Liganden-Interaktionen oder der Proteindynamik eingesetzt.

Im Rahmen der Arbeit wird ExPAN in Kombination mit einem neuen Ansatz in der Weitfeldmikroskopie untersucht, bei der die Orientierung von Farbstoffmolekülen als Kontrastmechanismus genutzt wird. Dabei wird die Polarisationsrichtung des Anregungslichts rotiert, um Informationen über die molekulare Orientierung zu gewinnen. Aufgrund der Photoselektion weist das Fluoreszenzsignal von Molekülen mit bevorzugter Ausrichtung dadurch eine periodische Modulation auf. Es wird gezeigt, dass diese Information zur Unterscheidung von Molekülen mit abweichender Orientierung genutzt werden kann, selbst wenn sich deren Signale räumlich überlagern. Für die Versuche wurde ein modifiziertes Weitfeld-Mikroskop konstruiert und die Methode zum einen experimentell an Einzelmolekülen und zum anderen mittels Simulationen erprobt. Dabei konnten Signale von Farbstoffmolekülen mit einem Abstand von bis zu $80 \mathrm{~nm}$ separiert werden. Darüber hinaus wurde ein moduliertes Fluoreszenzsignal bei oberflächenmarkierten Mikropartikeln in wässriger Lösung sowie bei fixierten biologischen Proben beobachtet. Eine Verbesserung der Photoselektion durch ExPAN wird experimentell nachgewiesen und gezeigt, dass mit ExPAN auch ähnlich orientierte Moleküle unterschieden werden können. 
Im zweiten Teil der Arbeit wird eine Methode zur Verbesserung der Auflösung von konfokalen Laser-Scanning-Mikroskopen vorgestellt, die als Multidetektor-Scanning (MDS) bezeichnet wird und auf dem Prinzip der Image-Scanning-Mikroskopie (ISM) beruht. Mit ISM lässt sich die Auflösung von Fluoreszenzmikroskopen theoretisch verdoppeln. Da ISM einen Flächendetektor voraussetzt, wurden in der Vergangenheit hauptsächlich CCD oder CMOS Kameras als Detektoren eingesetzt. In dieser Arbeit werden anstelle einer Kamera mehrere Einzelphotonendetektoren verwendet und über ein Glasfaserbündel zu einem Flächendetektor kombiniert. Dadurch ist es erstmals möglich, die Methode in Verbindung mit Fluoreszenzlebensdauer-Mikroskopie (FLIM) einzusetzen. FLIM hat sich in den Biowissenschaften als wichtige Mikroskopie-Technik etabliert und wird unter anderem bei ProteinProtein-Interaktionsstudien oder zur Untersuchung des NADH-Metabolismus eingesetzt. Die Verbesserung der räumlichen Auflösung von FLIM mit MDS ist somit für eine Reihe von biologischen Fragestellungen von potentiellem Interesse. Im Rahmen der Arbeit wurde ein Multidetektor-Scanning-Mikroskop konstruiert und durch die Vermessung von fluoreszierenden Mikropartikeln charakterisiert. Eine Verbesserung der Auflösung durch MDS wird an fixierten biologischen Proben demonstriert. Dabei wurde eine Auflösung von $(168 \pm 3) \mathrm{nm}$ mit MDS sowie $(146 \pm 3) \mathrm{nm}$ mit MDS und Dekonvolution erreicht. Schließlich wird die Kombination der Methode mit Fluoreszenzlebensdauer-Mikroskopie demonstriert. 


\section{Summary}

This doctoral thesis addresses two novel approaches in fluorescence microscopy.

The first part introduces a new method that can be used to enhance the effect of photoselection. This method, termed ExPAN (excitation polarization angle narrowing), exploits stimulated emission to narrow the angular distribution of excited fluorophores. ExPAN could be of significant interest for applications like fluorescence anisotropy measurements, which are commonly applied in life science, e.g. to study receptor-ligand interactions or investigate protein dynamics.

In the context of this work, ExPAN was applied in conjunction with a new fluorescence microscopy approach that utilizes molecular orientation as a contrast mechanism. Using excitation light with continuously rotating polarization, it is possible to obtain information about the orientation of dye molecules. When excited in this manner, molecules with a preferred orientation exhibit a periodically modulated fluorescence signal as a result of photoselection. This work showed that the additional modulation information can be utilized to distinguish molecules with different orientations even when their signals overlap spatially. The approach was explored by experiments with single molecules on a surface using a modified widefield microscope and additionally by simulation studies. The results indicated that it is possible to distinguish signals from dye molecules with a distance down to $80 \mathrm{~nm}$. It was demonstrated that ExPAN enhances the effect of photoselection and can be used to distinguish molecules even when their orientations are very similar. Furthermore, a modulated fluorescence signal was also observed in fixed biological samples and with surface labeled microparticles in aqueous solution.

In the second part of this thesis a method is presented for improving the resolution of confocal laser scanning microscopes. This method, termed multi-detector scanning (MDS), is based on the concept of image scanning microscopy (ISM). Using ISM it is theoretically possible to double the resolution of a fluorescence microscope. Since ISM requires an array detector, most of the previously developed implementations use CCD or CMOS cameras for detection. Instead of a camera, several single-photon detectors that are combined to an array 
detector by a fiber optic bundle, are used in this work. This configuration allowed it for the first time to use the method in conjunction with Fluorescence-lifetime imaging microscopy (FLIM). FLIM has proven to be an important microscopy technique in life science and has been applied in a wide variety of studies including protein-protein interaction and cell metabolism research. Therefore, the improvement in spatial resolution of FLIM through ISM is of potential interest for numerous biological applications. Within the scope of this work, a multi-detector scanning microscope was constructed and characterized. Resolution improvements of $(168 \pm 3) \mathrm{nm}$ with MDS and $(146 \pm 3) \mathrm{nm}$ with MDS and deconvolution were achieved in fixed biological samples. Furthermore, the measurement of fluorescence lifetimes was demonstrated with the experimental setup. Thus, the application of MDS has the potential to increase the spatial resolution of FLIM and could serve as a tool for measuring biological systems in more detail. 


\section{Abkürzungsverzeichnis}

\begin{tabular}{|c|c|}
\hline $\mathrm{APD}$ & avalanche photon diode \\
\hline BPAE & bovine pulmonary artery endothelial \\
\hline BSA & bovine serum albumin (Rinderalbumin) \\
\hline CCD & charge-coupled device \\
\hline CLSM & confocal laser scanning microscopy \\
\hline CSD & confocal spinning disk \\
\hline $\mathrm{CW}$ & continuous wave \\
\hline emCCD & electron multiplying charge-coupled device \\
\hline ExPAN & excitation polarization angle narrowing \\
\hline FLIM & fluorescence-lifetime imaging microscopy \\
\hline FWHM & full width at half maximum (Halbwertsbreite) \\
\hline IC & internal conversion \\
\hline ISC & intersystem crossing \\
\hline ISM & image scanning microscopy \\
\hline MDS & multi detector scanning \\
\hline MSIM & multifocal structured illumination microscopy \\
\hline MTF & Modulationsübertragungsfunktion \\
\hline OTF & optische Übertragungsfunktion \\
\hline PALM & photoactivated localization microscopy \\
\hline
\end{tabular}


PBS phosphate buffered saline

PMT photomultiplier tube

RL Richardson-Lucy

SIM structured illumination microscopy

SPAD single-photon avalanche diode

SPEED sparsity penalty enhanced estimation by demodulation

STED stimulated emission depletion

STORM stochastic optical reconstruction microscopy

TAC time to amplitude converter

TCSPC time-correlated single photon counting

TDC time to digital converter

TTL Transistor-Transistor-Logik 


\section{Vorwort}

Seitdem der niederländische Naturforscher Antoni van Leeuwenhoek vor etwa 400 Jahren das erste mal Mikroorganismen durch eine Linse beobachtete, ist die Lichtmikroskopie zu einem unersetzlichen Werkzeug für die biologische Forschung geworden. Im Laufe der Zeit wurden immerzu neue und verbesserte Methoden entwickelt, um Strukturen und Prozesse in biologischen Systemen sichtbar zu machen.

Als am Anfang des 20. Jahrhunderts spezialisierte Mikroskope eingeführt wurden, die mit ultraviolettem Licht arbeiteten, wurde häufig eine - eigentlich störende - Lumineszenz der Proben beobachtet. Diese Entdeckung führte in den folgenden Jahren zu der Entwicklung der Fluoreszenzmikroskopie durch Otto Heimstädt und Heinrich Lehmann [1, 2]. Obwohl Heimstädt zunächst nicht von der Bedeutung seiner Erfindung überzeugt war ${ }^{(1)}$, wurde die Fernfeld-Fluoreszenzmikroskopie zu einem der wichtigsten bildgebenden Verfahren der Biowissenschaften [1].

Die Entwicklung der Immunfluoreszenz in den 40er Jahren [4] ermöglichte die gezielte Abbildung von Proteinen durch fluoreszenzmarkierte Antikörper und führte zu bedeutenden Fortschritten auf dem Gebiet der Zellbiologie [5]. Als großer Vorteil gegenüber anderen Mikroskopieverfahren erwies sich dabei die hohe Selektivität und der hohe Kontrast mit der unterschiedliche Strukturen in einer Zelle visualisiert werden konnten [1].

Die Erfindung des Lasers und neuer Photodetektoren ermöglichten die Markteinführung des ersten konfokalen Laser-Scanning-Mikroskops in den 1980er Jahren durch die Firma Carl Zeiss [1]. Dabei wird die Probe durch einen Lichtpunkt rasterförmig abgetastet, wodurch Hintergrundlicht unterdrückt und eine bessere Auflösung erreicht werden konnte. Im Folgenden wurde dieses Konzept durch die Anregung über Zwei-Photonen-Absorption [6] erweitert, mit der es möglich wurde scharfe Bilder auch in tiefen Schichten einer dreidimensionalen Probe aufzunehmen $[7,8]$. Ein weiterer großer Fortschritt bei der Fluoreszenzmarkierung von biologischen Proben gelang in den 90er Jahren durch das Fluoreszenzprotein GFP (green

\footnotetext{
(1) In seiner Publikation über das Fluoreszenzmikroskop resümiert er: „Ob und wieweit das Fluoreszenzmikroskop im besonderen und die Lumineszenzmikroskope im allgemeinen eine Möglichkeit der Erweiterung des mikroskopischen Abbildungsgebiets in sich schließen, muß die Zukunft lehren." [3]
} 
fluorescence protein) [9]. Fluoreszenzproteine können direkt im Genom eines Organismus codiert werden, sodass sich fast alle Proteine einer lebenden Zelle gezielt fluoreszenzmarkieren lassen.

Diese Arbeit beschäftigt sich mit zwei neuen methodischen Ansätzen aus dem Gebiet der Fluoreszenzmikroskopie. Im ersten Teil wird untersucht in wie weit die Orientierung von Farbstoffmolekülen als Kontrastmechanismus in der Fluoreszenzmikroskopie genutzt werden kann. Der zweite Teil der Arbeit beschäftigt sich mit einer Methode zur Verbesserung der Auflösung von konfokalen Laser-Scanning-Mikroskopen, bei der das Fluoreszenzlicht gleichzeitig durch mehrere Detektoren erfasst wird. 


\section{Molekulare Orientierung als Kontrastmechanismus in der Fluoreszenzmikroskopie}

\section{$1.1 \quad$ Einleitung}

Die dreidimensionale Ausrichtung von einzelnen Farbstoffmolekülen ist neben der Fluoreszenzlebensdauer und dem Emissionsspektrum von besonderem Interesse für die Einzelmolekülspektroskopie [10, 11]. Beispielsweise gibt die Orientierung von fluoreszenzmarkierten Proteinen wichtige Einblicke in die Funktionsweise von molekularen Motoren. Auf diesem Weg konnten unter anderem die Rotation der $\mathrm{F}_{1}$-ATPase $[12,13]$ und der Bewegungsablauf des Motorproteins Kinesin [14, 15] untersucht werden.

Die relative Orientierung eines Moleküls wirkt sich maßgeblich auf dessen Wechselwirkung mit einem elektromagnetischen Feld aus. Entscheidend für die Anregung und Emission von Fluoreszenz ist dabei das elektronische Übergangsdipolmoment eines Moleküls, das durch dessen molekulare Struktur vorgegeben ist. Betzig et. al konnten als erste die Orientierung des Übergangsdipolmoments einzelner Moleküle durch optische Rasternahfeldmikroskopie bestimmen [16]. Die Messung im Fernfeld wurde kurze Zeit später beschrieben [17, 18] und konnte im Folgenden mit unterschiedlichen Methoden und Mikroskopietechniken realisiert werden. Dabei lässt sich grundsätzlich zwischen zwei verschiedenen Ansetzten unterscheiden: Durch das Einbringen von Polarisationsoptiken in Weitfeld- oder Konfokal-Mikroskope lässt sich die Polarisationsabhängigkeit der Anregung ausnutzen, um Informationen über die Orientierung des Übergangsdipolmoments von Molekülen zu gewinnen. Alternativ, oder in Kombination, kann dazu auch die Polarisation der Fluoreszenzemission vermessen werden. Dieser Ansatz wurde in einer Reihe von Arbeiten verfolgt [17-23] und ist eine robuste Methode, um die Orientierung von Farbstoffmolekülen innerhalb der Fokusebenen eines Fluoreszenz- 
mikroskops zu bestimmen. Die genaue dreidimensionale Ausrichtung ist auf diesem Weg allerdings nicht direkt zugänglich und erfordert komplexere Versuchsaufbauten [24, 25].

Weitere Arbeiten nutzen die Intensitätsverteilung der Abbildung einzelner Fluoreszenzfarbstoffe, um Informationen über deren Ausrichtung zu gewinnen [26-30]. Ein einzelnes, fluoreszierendes Molekül verhält sich wie ein Hertz'scher Dipol, bei dem die Amplitude der abgestrahlten elektromagnetischen Welle richtungsabhängig ist. Bei der Abbildung eines fixierten Moleküls entsteht dadurch eine anisotrope Intensitätsverteilung in der Bildebene, die neben der dreidimensionalen Orientierung des Übergangsdipolmoments von den Eigenschaften des Abbildungssystems und dem Abstand des Moleküls zu der Fokusebene abhängt. Eine geeignete physikalische Beschreibung des Abbildungsprozesses erlaubt die Rekonstruktion der Ausrichtung des Übergangsdipolmoments einzelner Moleküle anhand der gemessenen Intensitätsverteilung [27]. Darüber hinaus konnte in jüngeren Arbeiten gezeigt werden, dass durch bestimmte Modifikationen des Abbildungssystems Muster in der Bildebene erzeugt werden können, die ebenfalls von der Orientierung der Emitter abhängig sind [31].

Im Gegensatz zu den genannten Publikationen liegt der Fokus in diesem Teil Arbeit nicht auf der exakten Bestimmung der Orientierung einzelner Fluoreszenzfarbstoffe. Vielmehr wird sich der Frage gewidmet, ob eine Unterscheidung von Farbstoffmolekülen und fluoreszenzmarkierter Strukturen anhand der molekularen Orientierung möglich ist, selbst wenn sich deren Fluoreszenzsignal räumlich überlagert. Die Auswertung der Intensitätsverteilung einzelner Emitter zur Orientierungsbestimmung ist für diesen Zweck nicht geeignet. Aus diesem Grund wird in dieser Arbeit die Polarisationsabhängigkeit der Fluoreszenzanregung ausgenutzt, um Informationen über die Ausrichtung der Moleküle zu gewinnen. Hierfür wurde ein Weitfeldmikroskop konstruiert bei dem die Polarisation des Anregungslichts moduliert werden kann. Durch eine Polarisationsoptik wird die Richtung des linear polarisierten Anregungslichts kontinuierlich rotiert. Bei einer Probe mit einer konstanten Ausrichtung der Fluoreszenzemitter, oder zumindest eine entsprechenden Vorzugsrichtung, führt das zu einer sinusoidalen Modulation der gemessenen Fluoreszenzintensität. Auf diesem Weg können für jeden Bildpunkt, neben der Gesamtintensität, zusätzliche Informationen in Form von Phase und Amplitude der Modulation gewonnen werden. Die Phase der Intensitätsmodulation ist dabei abhängig von der Orientierung der Fluoreszenzemitter. Prinzipiell sollte diese zusätzliche Information genutzt werden können, um Emitter mit abweichender Ausrichtung zu unterscheiden. Allerdings ist die Winkelverteilung der Anregungswahrscheinlichkeit relativ unspezifisch. Signale von Emittern die sich in räumlicher Nähe befinden und ähnliche Orientierung aufweisen sind aus diesem Grund schwer zu trennen. Als Lösung für dieses Problem wird eine Methode vorgestellt, mit der es möglich ist den Winkelbereich der Fluoreszenzanregung zu verkleinern. Die Methode wird als ExPAN (excitation polarization angle narrowing) 
bezeichnet und basiert auf der Fluoreszenzlöschung durch stimulierte Emission. Dabei wird eine zweite Lichtquelle mit senkrechter Polarisation zum Anregungslicht eingesetzt.

Für die Auswertung der so gewonnen Daten wurde ein Bildrekonstruktionsalgorithmus verwendet, der die zusätzlichen Informationen aus der Modulation berücksichtigt. Der experimentelle Aufbau und die Datenauswertung wurden an einzelnen Farbstoffmolekülen auf einer Glasoberfläche erprobt. Zusätzlich wurden Daten simuliert, um die Auswirkung der Farbstoffdichte und ExPAN auf die Datenauswertung zu untersuchen. Schließlich wurde untersucht in wie weit eine Modulation der Fluoreszenzintensität auch bei komplexeren und biologischen Proben zu beobachten ist. 


\subsection{Theoretische Grundlagen}

\subsubsection{Absorption und das Übergangsdipolmoment}

Durch Absorption von elektromagnetischer Strahlung kann ein Molekül in einen elektronisch angeregten Zustand wechseln. Im einfachsten Fall geht ein Molekül von dem elektronischen Grundzustand $\mathrm{S}_{0}$ in den ersten angeregten Singulett-Zustand $\mathrm{S}_{1}$ über. Zwei verschiedene elektronische Zustände zeichnen sich dabei durch eine unterschiedliche Verteilung der Ladungsdichte aus. Um einen Übergang vom Grundzustand in einen angeregten Zustand zu bewirken, muss das oszillierende elektrische Feld eine Umverteilung der Ladungsdichte induzieren. Die Änderung der Ladungsdichte wird durch das Übergangsdipolmoment charakterisiert. Für den Übergang von dem Ausgangszustand $A$ zu dem Endzustand $E$ gilt für das Übergangsdipolmoment [32]

$$
\vec{\mu}_{E A}=\int \psi_{E}^{*} \hat{\mu} \psi_{A} d \tau
$$

Hier sind $\psi_{A}$ und $\psi_{E}$ die Wellenfunktion des Ausgangs- und Endzustands. $\hat{\mu}$ ist der Operator des elektrischen Dipolmoments, der sich aus dem Ort der Elektronen $\vec{r}$ und dem der Kerne $\vec{R}$ mit der den Ladungen $e$ bzw. $Z$ zusammensetzt,

$$
\hat{\mu}=-e \sum_{i} \vec{r}_{i}+e \sum_{j} Z_{j} \vec{R}_{j}
$$

Die Gesamtwellenfunktion lässt sich unter Annahme der Born-Oppenheimer-Näherung als Produkt der zugehörigen elektronischen Wellenfunktion $\psi_{\epsilon}$ und der Schwingungswellenfunktion $\psi_{v}$ beschreiben. Da die Dynamik der leichten Elektronen um Größenordnungen schneller ist als die der schweren Atomkernen, lassen sich diese in guter Näherung getrennt voneinander betrachten. Das Übergangsdipolmoment kann unter dieser Annahme als Produkt von zwei Beiträgen geschrieben werden,

$$
\vec{\mu}_{E A}=\vec{\mu}_{\epsilon E A} S\left(v_{E}, v_{A}\right)
$$

mit

$$
\vec{\mu}_{\epsilon E A}=-e \sum_{i} \int \phi_{\epsilon E}^{*} \vec{r}_{i} \phi_{\epsilon A} d \tau_{\epsilon} \quad \text { und } \quad S\left(v_{E}, v_{A}\right)=\int \psi_{v E}^{*} \psi_{v A} d \tau_{v} .
$$

$S\left(v_{E}, v_{A}\right)$ ist das Überlappungsintegral zwischen der Schwingungswellenfunktion des angeregten und des Grundzustands. Bei der Absoption eines Photons kann ein Molekül neben der elektronischen Anregung auch in einen angeregten Schwingungszustand übergehen. Die Wahrscheinlichkeit eines Überganges zwischen den beide Schwingungszuständen $v_{A}$ und $v_{E}$ ist dabei proportional zu dem Quadrat des Überlappungsintegrals $S\left(v_{E}, v_{A}\right)^{2}$ und wird als 


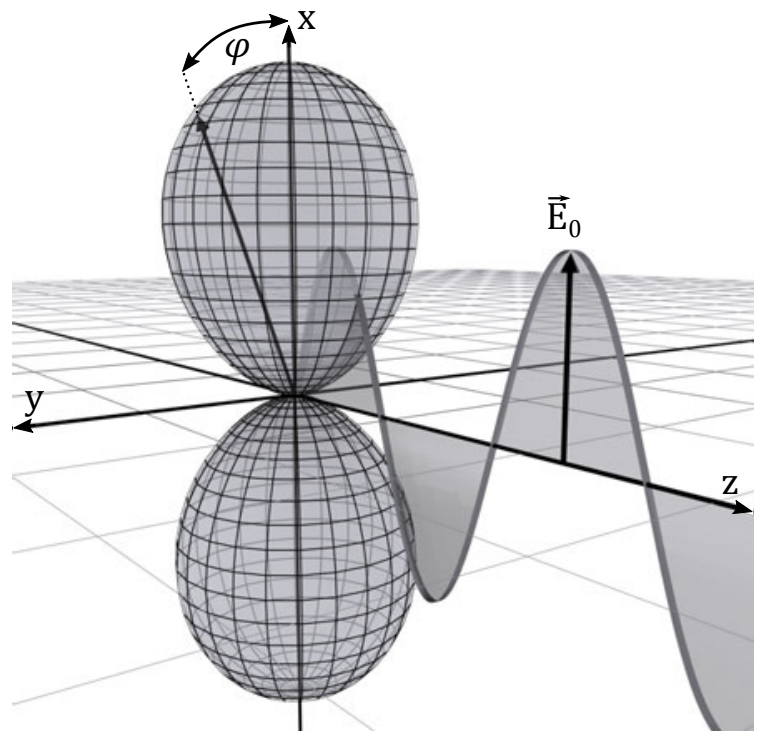

\section{Abbildung 1.1:}

Übergangswahrscheinlichkeit für einen elektrischen Feldvektor $\vec{E}_{0}$ parallel zur $\mathrm{x}$-Achse und den möglichen Orientierungen des Übergangsdipolmoments. Die Übergangswahrscheinlichkeit ist proportional $\mathrm{zu}$ der Länge des Vektors vom Ursprung zur der abgebildeten Fläche mit der Funktion $P=\cos ^{2}(\varphi)$.

Franck-Condon-Faktor bezeichnet. In den meisten Fällen ist der Franck-Condon-Faktor für Übergänge vom Grundzustand in einen moderat angeregten Schwingungszustand am größten, was zu der charakteristischen Form vieler Absorptionsspektren führt [32].

Das elektronische Übergangsdipolmoment $\vec{\mu}_{\epsilon E A}$ ist ein Maß für die Umverteilung der Elektronendichte bei dem Übergang zwischen den elektronischen Zuständen und ist von zentraler Bedeutung bei der Absorption und Emission von elektromagnetischer Strahlung. Die Wahrscheinlichkeit der Absorption ist quadratisch proportional zum Skalarprodukt des elektrischen Feldvektors $\vec{E}_{0}$ und dem elektronischen Übergangsdipolmoment,

$$
P_{A \rightarrow E} \propto\left(\vec{E}_{0} \cdot \vec{\mu}_{\epsilon E A}\right)^{2}=\left\|\vec{E}_{0}\right\|^{2}\left\|\vec{\mu}_{\epsilon E A}\right\|^{2} \cos ^{2}(\varphi)
$$

oder

$$
P_{A \rightarrow E} \propto \cos ^{2}(\varphi)
$$

Je größer die Komponente von $\vec{\mu}_{\epsilon E A}$ in Richtung dieses Feldvektors ist, umso wahrscheinlicher wird der entsprechende Übergang angeregt. Demzufolge hängt die Übergangswahrscheinlichkeit vom Winkel $\varphi$ zwischen dem Übergangsdipolmement und dem elektrischen Feldvektor ab und wird maximal wenn $\varphi=0$ (siehe Abbildung 1.1). Bei linear polarisiertem Licht haben der elektrische Feldvektor und der Vektor der Polarisation die gleiche Orientierung, in dem Fall entspricht $\varphi$ dem Winkel zwischen dem Übergangsdipolmoment und dem Vektor der Polarisation. Wird eine Ensemble von Molekülen mit isotroper Orientierung durch linear polarisiertes Licht angeregt, dann ist die Winkelverteilung der Population angeregter Moleküle ebenfalls proportional zu $\cos ^{2}(\varphi)$. Dieses Phänomen wird Photoselektion genannt $[32,34]$. 
Abbildung 1.2: Jablonski-Diagramm mit den möglichen Relaxationspfaden nach einer Anregung in den Singulett-Zustand $\mathrm{S}_{1}$. Geschwungene Linien stellen strahlungslose Übergänge dar.

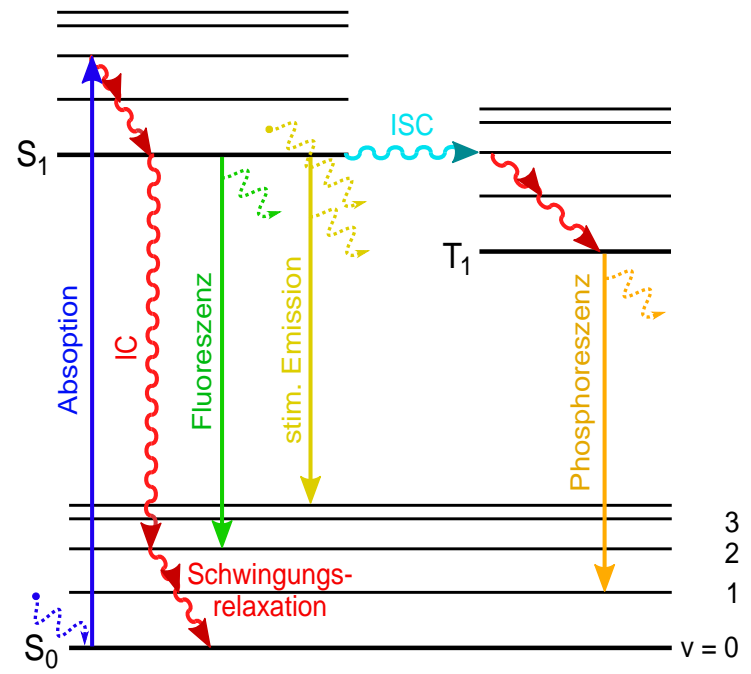

\subsubsection{Relaxation elektronisch angeregter Zustände}

Nach dem Frank-Condon-Prinzip [33] ist die elektronische Anregung eines Moleküls in der Regel auch mit der Anregung in einen höheren Schwingungszustand verbunden. Die Relaxation in den Schwingungsgrundzustand geschieht bei Molekülen in flüssiger Phase schnell $\left(\sim 10^{-12} \mathrm{~s}\right)$ und strahlungslos durch Stöße mit dem umgebenden Lösungsmittel [34]. Die anschließende Rückkehr in den elektronischen Grundzustand kann durch verschiedene Relaxationsprozesse erfolgen (siehe Abbildung 1.2).

Durch innere Umwandlung (Internal Conversion, IC) kann ein Molekül Strahlungslos in den Grundzustand relaxieren. Dabei wird die Energie des angeregten Zustands auf höhere Schwingungszustände eines tiefer liegenden elektronischen Niveaus übertragen und an das umgebene Lösungsmittel abgegeben. Die innere Umwandlung ist an einem Schnittpunkt der Potentialkurven der jeweiligen Zustände am wahrscheinlichsten [35].

Der Relaxation aus einem angeregten Singulet-Zustand durch Emission eines Photons wird Fluoreszenz genannt. Bei Molekülen sind die Emissionsbanden nicht scharf, sondern wie bei der Absorption mit der Schwingungsstruktur der jeweiligen Zustände überlagert. Da bei der Schwingungsrelaxation Energie an das umgebende Medium abgegeben wird, hat das emittierte Photon eine geringere Energie als das absorbierte. Zusätzlich wird durch die veränderte Ladungsverteilung eine Reorganisation der umgebenden Lösungsmittelmoleküle induziert, wodurch die Energie ebenfalls gesenkt wird. Die resultierende Rotverschiebung des Emissionsspektrums wird als Stokes-Verschiebung bezeichnet. Moleküle bei denen Fluoreszenzemission der dominante Relaxationsprozess ist, und die damit eine hohe Fluoreszenzquantenausbeute aufweisen, werden Fluorophore genannt. Viele Fluorophore werden 
durch Absorption in das $\mathrm{S}_{2}$-Niveau angeregt, relaxieren durch Internal Conversion in das $\mathrm{S}_{1}$-Niveau und kehren von dort durch Fluoreszenzemission in den Grundzustand zurück [34]. Auch durch die Wechselwirkung mit Licht passender Wellenlänge kann ein elektronisch angeregtes Molekül in den Grundzustand zurückkehren [36]. Dieser Prozess wird stimulierte Emission genannt und ist von dem Übergangsdipolmoment und der Intensität der elektromagnetischen Strahlung abhängig. Durch stimulierte Emission wird ein Photon erzeugt, das die selben Eigenschaften besitzt wie das eingestrahlte Photon.

Des Weiteren ist der Übergang von dem $\mathrm{S}_{1}$-Niveau in den ersten Triplett-Zustand $\mathrm{T}_{1}\left(\mathrm{~S}_{1} \rightarrow\right.$ $\mathrm{T}_{1}$ ) durch Spinumkehr möglich. Dieser eigentlich spinverbotene Übergang wird Intersystem Crossing (ISC) genannt. Durch schwerere Elemente mit größerer Spin-Bahn-Kopplung kann das Spinverbot abgeschwächt und der Übergang begünstigt werden. Aus dem $\mathrm{T}_{1}$-Niveau kann das System durch Emission eines Photons in den in den Grundzustand zurückkehren $\left(\mathrm{T}_{1} \rightarrow \mathrm{S}_{0}\right)$. Die Emission aus dem Triplett-Zustand wird Phosphoreszenz genannt. In der Regel ist das Phosphoreszenz gegenüber der Fluoreszenz rotverschoben. Aufgrund des Spinverbots sind die Lebensdauern im Triplett-Zustand deutlich größer $\left(\sim 10^{-4}-10^{4} \mathrm{~s}\right)$ als bei der Fluoreszenz $\left(\sim 10^{-10}-10^{-7} \mathrm{~s}\right)$.

\subsubsection{Weitfeld-Fluoreszenzmikroskopie}

Die Abbildung eines Objekts durch ein Mikroskop setzt voraus, dass es einen Kontrastmechanismus gibt, der das Objekt von der Umgebung unterscheidbar macht. Dazu können verschiedene Wechselwirkungen des Lichts mit der Probe genutzt werden. Gängige Mikroskopie-Techniken verwenden Phasenverschiebung, Streuung oder Absorption des eingestrahlten Lichts. Die Kontrastbildung von Fluoreszenzmikroskopen basiert auf der Stokes-Verschiebung zwischen Anregungs- und Emissionslicht. Das Emissionslicht kann aufgrund der unterschiedlichen Wellenlänge mit optischen Filtern von dem Anregungslicht separiert werden, sodass nur das Licht detektiert wird, welches von Fluoreszenzfarbstoffen in Probe emittiert wurde.

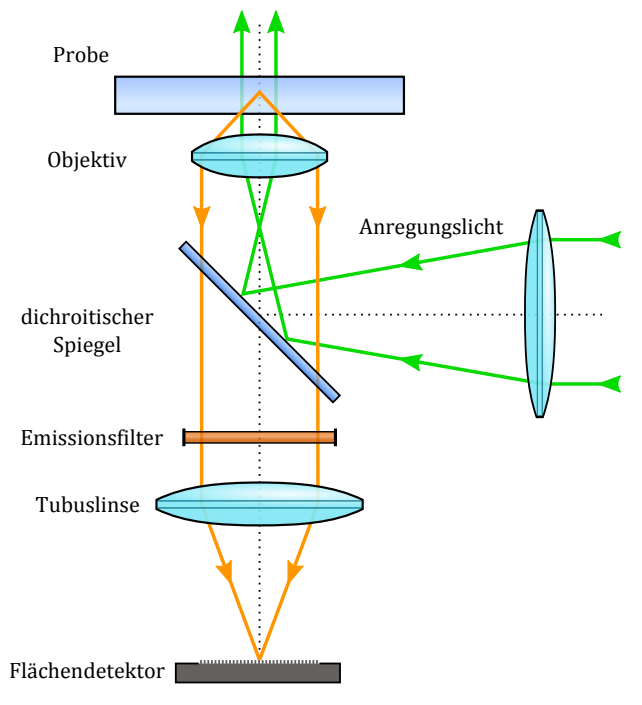

Abbildung 1.3: Schematischer Aufbau eines Weitfeld-Fluoreszenzmikroskops. 
Abbildung 1.4: Ideale Abbildung eines Punktemitters durch zwei Linsen.

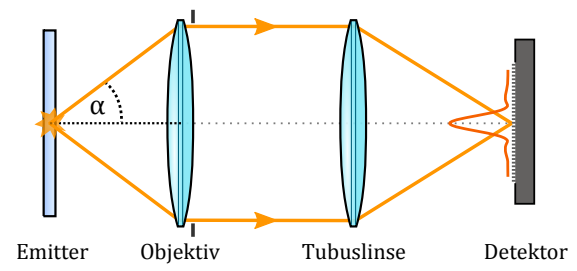

Moderne Mikroskope arbeiten in der Regel mit einem Unendlichstrahlengang, bei dem Objekt- und Fokusebene des Objektivs identisch sind und das Bild durch eine Tubuslinse erzeugt wird. Die Vergrößerung ist dadurch unabhängig von der Distanz zwischen dem Objektiv und der Tubuslinse. Weitere optische Komponenten, wie z.B. Fluoreszenzfilter, lassen sich so flexibel in dem dazwischenliegenden Strahlengang installieren. WeitfeldFluoreszenzmikroskope werden in den meisten Fällen als Auflichtmikroskope konstruiert. Dabei wird das Anregungslicht auf die Rückapertur des Objektives fokussiert (siehe Abbildung 1.3) und regt Fluoreszenz in dem gesamten Bildbereich der Probe an. Das Fluoreszenzlicht wird von dem selben Objektiv gesammelt, mit optischen Filtern vom Anregungslicht getrennt und durch die Tubuslinse auf einen Flächendetektor abgebildet. Ein Vorteil gegenüber einer konfokalen Detektion besteht, neben dem relativ simplen optischen Aufbau, vor allem in einer deutlich höheren Zeitaufösung. Aufgrund der parallelen Abbildung des gesamten Bildbereichs sind Aufnahmen mit Videogeschwindigkeit und schneller möglich. Allerdings gibt es keine räumliche Filterung des detektierten Lichts, wodurch Hintergrundlicht und Licht von außerhalb der Fokusebene ungehindert den Detektor erreichen kann. Aus diesem Grund ist insbesondere die axiale Aufösung geringer als bei einer konfokalen Detektion.

\subsubsection{Abbildungseigenschaften eines Weitfeld-Fluores- zenzmikroskops}

Die Bildentstehung in einem Weitfeld-Mikroskop lässt sich vereinfacht anhand eines Systems aus zwei idealen Linsen (Objektivlinse und Tubuslinse), einer Objektebene und einer Bildebene beschreiben (siehe Abbildung 1.4). Wird ein punktförmiger, inkohärenter Emitter in der Objektebene - in guter Näherung z.B. ein einzelnes Farbstoffmolekül - durch das Linsensystem abgebildet, ist die resultierende Intensitätsverteilung in der Bildebene kein perfekter Punkt, sondern eine sog. Beugungs- oder Airy-Scheibe. Die Objektivlinse besitzt einen begrenzten Durchmesser und kann daher nur einen Teil des Lichts aus der Objektebene einfangen. Wenn $\alpha$ den halbe Öffnungswinkel des Objektives darstellt, wird nur Licht innerhalb eines Kegels mit dem Öffnungswinkel $2 \alpha$ von dem Objektiv erfasst (siehe Ab- 
bildung 1.4). Dadurch wirkt die Apertur des Objektives als Lochblende an der es zu sog. Frauenhofer-Beugung kommt. Die räumliche Ausbreitung der Intensitätsverteilung in der Bildebene ist letztendlich Folge der Beugung an der Apertur des Objektives. Mithilfe des Beugungsintegrals lässt sich die komplexe Amplitude des Lichts in der Bildebene mathematisch beschreiben. In der hier relevanten Frauenhofer-Näherung entspricht das Beugungsintegral der inversen Fourier-Transformation der Blendenfunktion $f_{S}[37,38]$,

$$
U(\vec{x}) \propto \int f_{S}(\vec{s}) e^{i k \vec{x} \cdot \vec{s}} d S
$$

Hier ist $U(\vec{x})$ die Amplitude in der Bildebene am Punkt $\vec{x}, k=\frac{2 \pi}{\lambda}$ der Betrag des Wellenvektors und $\vec{s}$ ein Punkt in der Apertur. Die Blendenfunktion beschreibt die Form der Apertur, im Normalfall eine Kreisfläche. Für eine kreisförmige Apertur ist es dabei sinnvoll den Ausdruck direkt in Polarkoordinaten zu formulieren:

$$
\begin{gathered}
\vec{x}=\left(\begin{array}{l}
q \cos \varphi \\
q \sin \varphi
\end{array}\right), \vec{s}=\left(\begin{array}{c}
r \cos \theta \\
r \sin \theta
\end{array}\right), d S=r d r d \theta \\
U(q, \varphi) \propto \int_{0}^{a} \int_{0}^{2 \pi} e^{i k q r \cos (\theta-\varphi)} r d \theta d r
\end{gathered}
$$

Das innere Integral in Ausdruck 1.8 entspricht der Integraldarstellung der Besselfunktion erster Gattung $J_{0}{ }^{(2)}$ und lässt sich mithilfe von Rekursionsbeziehungen ${ }^{(3)}$ weiter vereinfachen $\mathrm{zu}[37]$

$$
U(q) \propto \frac{2 J_{1}(k \mathrm{NA} q)}{k \mathrm{NA} q} .
$$

Wobei hier die nummerische Apertur der Objektivlinse NA $=n \sin \alpha$ eingeführt wurde, die sich aus dem Produkt des Brechungsindexes des Immersionsmediums $n$ und dem Sinus des halben Öffnungswinkels des Objektives ergibt. Weiterhin lässt sich eine unabhängige optische Einheit $v$ für den lateralen Abstand von der optischen Achse einführen:

$$
v=k \mathrm{NA} q=\frac{2 \pi}{\lambda} n \sin (\alpha) \sqrt{x^{2}+y^{2}}
$$

Aus dem Betragsquadrat der Amplitude ergibt sich dann die reelle Intensität

$$
I(v) \propto\left|\frac{2 J_{1}(v)}{v}\right|^{2} .
$$

Der Ausdruck 1.11 beschreibt das Beugungsmuster in der Bildebene, das durch einen punktförmigen Emitter entsteht. In Abbildung 1.5 ist die korrespondierende Intensitätsverteilung abgebildet, die der sog. Punktspreizfunktion (engl. point spread function, PSF) eines idealen Abbildungssystems entspricht. Hier wurde jedoch nur der Fall betrachtet, bei dem sich

\footnotetext{
${ }^{(2)}$ Integraldarstellung der Besselfunktion 0-ter Ordnung: $\frac{1}{2 \pi} \int_{0}^{2 \pi} e^{i x \cos \alpha} d \alpha=J_{0}(x)$

(3) Es gilt: $\int_{0}^{x} x^{\prime} J_{0}\left(x^{\prime}\right) d x^{\prime}=x J_{1}(x)$
} 
Emitter und Detektor im Fokus befinden. Die vollständige PSF ist eine Funktion über den dreidimensionalen Raum und gibt auch die Intensitätsverteilung für einen defokussierten Punktemitter wieder.

Durch die PSF werden die Abbildungseigenschaften eines optischen Systems weitgehend charakterisiert. Wird die Dichte im Objektraum durch eine Funktion $k(\vec{r})$ beschrieben und durch ein optisches System mit der Punktspreizfunktion $U(\vec{r})$ abgebildet, so lässt sich die resultierende Intensität in der Bildebene $I(\vec{r})$ durch die Faltung von $k(\vec{r})$ mit der PSF berechnen:

$$
I(\vec{r})=(k * U)(\vec{r})=\int k\left(\vec{r}^{\prime}\right) U\left(\vec{r}-\vec{r}^{\prime}\right) d \vec{r}^{\prime}
$$

Zudem lässt sich das Auflösungsvermögen direkt aus der PSF ableiten, wobei hier allerdings unterschiedliche Kriterien angewendet werden können. Entsprechend dem RayleighKriterium sind zwei Punkte in einer Abbildung gerade noch unterscheidbar, wenn das Maximum der Beugungsscheibe des einen Punkts in das erste Minimum der Beugungsscheibe des zweiten Punkts fällt [39]. Für ein beugungsbegrenztes Abbildungssystem ist das der Fall, wenn der Abstand der beiden Punkte

$$
d_{R}=0.61 \frac{\lambda}{\mathrm{NA}}
$$

beträgt. Bei experimentellen Daten ist es in der Regel vorteilhaft die Halbwertsbreite (FWHM) der PSF zu bestimmen, die ebenfalls als Maß für das Aufösungsvermögen benutzt werden kann. Für die Halbwertsbreite der Beugungsscheibe gilt [40],

$$
\mathrm{FWHM}=0.52 \frac{\lambda}{\mathrm{NA}}
$$

Die Fourier-Transformierte der PSF wird als optische Übertragungsfunktion (engl. optical transfer function, OTF) bezeichnet. Sie gibt die Übertragung von Ortsfrequenzen aus der Objektebene in der Bildebene an. Die Betrag der OTF wird als Modulationsübertragungsfunktion (MTF) bezeichnet und beschreibt die Übertragung der Amplitude. Die MTF für

Abbildung 1.5: PSF eines idealen Abbildungssystems für ein Punktemitter im Fokus $(z=0)$, gemäß Ausdruck 1.11. Auf der rechten Seite wurde der Weißwert des Bildes verringert, um die Ringe der Beugungsscheibe sichtbar zu machen. Achseneinteilung in dimensionslosen optischen Einheiten.

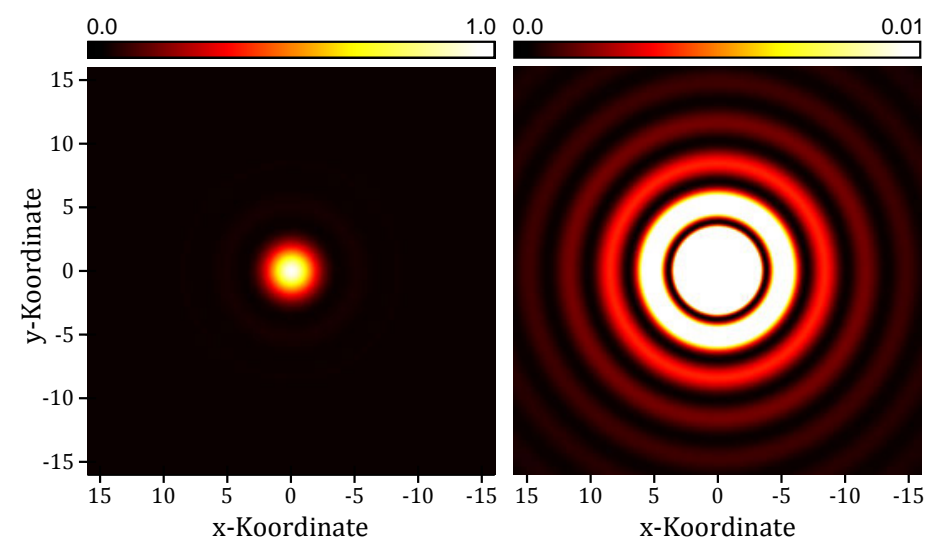



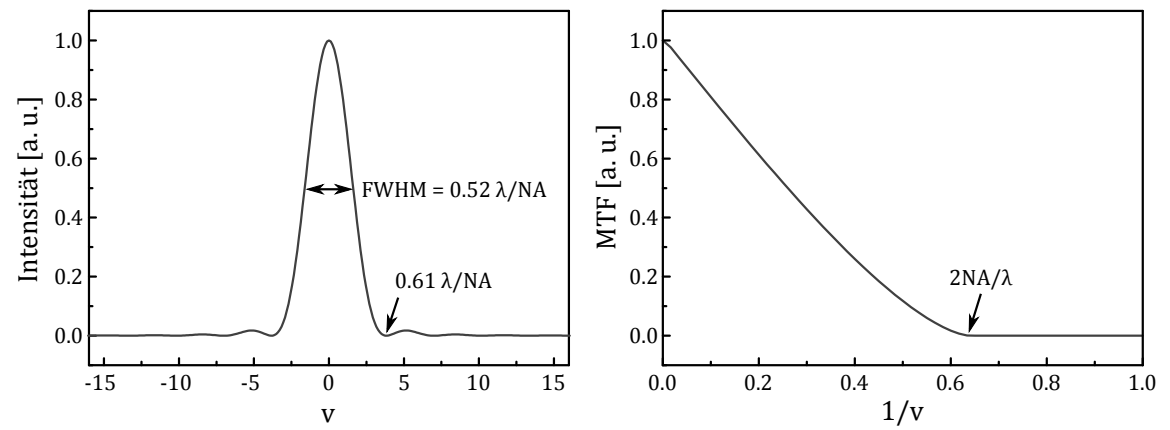

Abbildung 1.6: Links: Intensitätsprofil der PSF eines beugungsbegrenzten Abbildungssystems in lateraler Richtung $(y=z=0)$, aufgetragen gegen die dimensionslose optische Einheit $v$. Markiert sind die Halbwertsbreite sowie die Position des ersten Minimus. Rechts: Verlauf der Modulationsübertragungsfunktion eines idealen Abbildungssystems aufgetragen gegen $1 / v$.

ein beugungsbegreztes Abbildungssystem ist in Abbildung 1.6 aufgetragen. Der Verlauf der MTF zeigt, dass eine optische Abbildung den Charakter eines Tiefpassfilters besitzt, der höhere Frequenzen in der Objektebene mit zunehmender Dämpfung in die Bildebene überträgt. Die Grenzfrequenz, ab der keine weiteren Informationen mehr von der Objektebene in die Bildebene übertragen werden, entspricht der Auflösungsgrenze nach Ernst Abbe, auch bekannt als Abbe-Limit [41]. Das Abbe-Limit besagt, dass ein periodisches Gitter nur dann durch ein optisches Mikroskop aufgelöst werden kann, wenn der Linienabstand größer ist als

$$
d_{A}=\frac{\lambda}{2 \cdot \mathrm{NA}} .
$$

In einem realen optischen System wird die PSF zusätzlich durch das Auftreten von Aberrationen beeinflusst. Dabei führen insbesondere unterschiedliche Brechungsindices zwischen der Probe und dem Immersionsmedium zu spährischen Aberrationen, die sich in der Form der PSF widerspiegeln. 


\subsection{Experimentelles}

\subsubsection{Experimenteller Aufbau}

Der im Rahmen des Projekts konstruierte Aufbau entspricht zum größten Teil einem Weitfeld-Epifluoreszenzmikroskop. Als Basis für den Aufbau wird ein modifizierter IX 50-Mikroskop-Körper der Firma Olympus verwendet. Dabei handelt es sich um ein inverses Mikroskopsystem, das leicht durch zusätzlichen Komponenten erweitert werden kann. Der Strahlengang des Aufbaus ist schematisch in Abbildung 1.7 dargestellt. Für die Fluoreszenzanregung wird ein CW-Dioden-Laser (Sapphire 568 LP, Coherent) mit einer Wellenlänge von $568 \mathrm{~nm}$ verwendet. Der Strahldurchmesser des Lasers wird über ein Linsenpaar (L1, L2) auf circa $10 \mathrm{~mm}$ aufgeweitet und passiert im Folgenden einen variablen Polarisationsfilter $(\mathrm{P})$, der zur Einstellung der Anregungsleistung verwendet wird. Das vertikal polarisierte Anregungslicht wird anschließend durch einen polarisierenden Strahlteilerwürfel (PS; PBS101, Coherent) umgelenkt, der es ermöglicht einen zweiten Laser mit horizontaler Polarisation einzukoppeln und zudem die Qualität der Polarisation verbessert. Im Anschluss passiert der Anregungsstrahl zwei identische achromatische lambda-viertel Plättchen (WP1, WP2; AQWP05M-600, Thorlabs), die zur Rotation des linear polarisierten Lichts verwendet werden. Beide Plättchen können um die optische Achse gedreht werden, wobei eines der Plättchen (WP2) in einer kugelgelagerten Aufhängung fixiert ist und über einen Zahnriemen von einen Elektromotor angetrieben wird. Nach passieren des ersten Plättchens hat das Anregungslicht eine

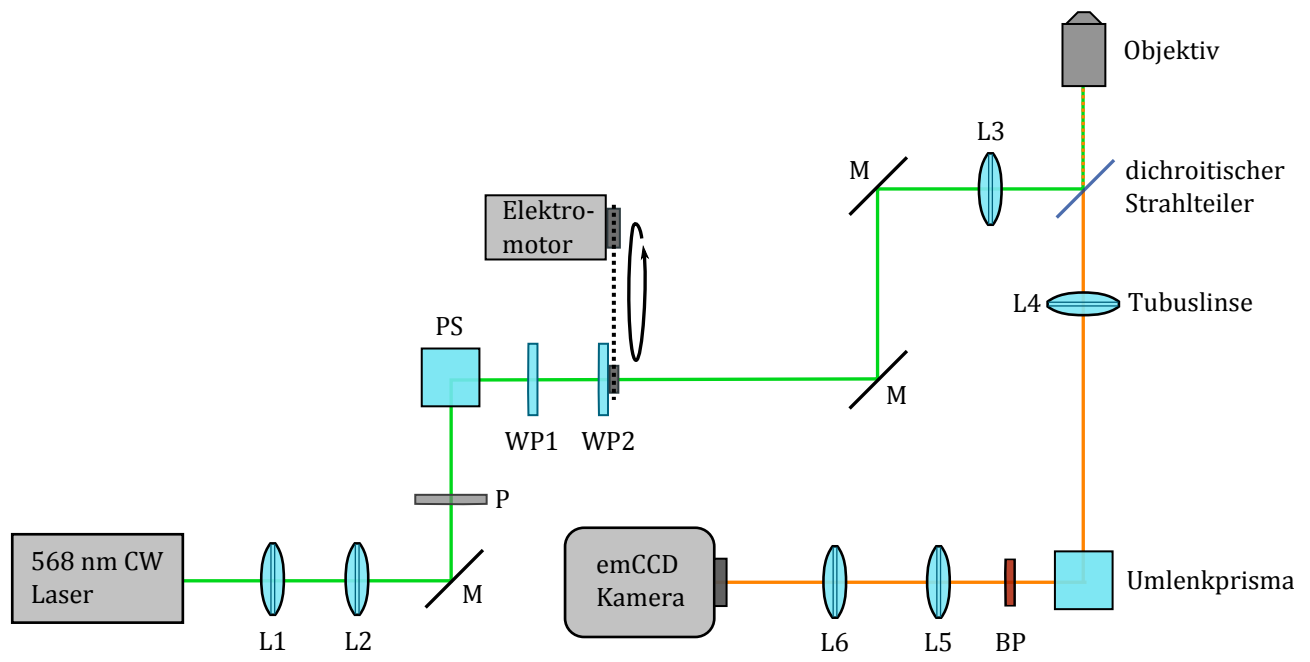

Abbildung 1.7: Illustration des optischen Aufbaus. M Spiegel, L Linse, PS polarisierender Strahlteilerwürfel, WP lambda-viertel Plättchen, P Polarisationsfilter, BP Bandpassfilter. 
zirkulare Polarisation, die durch das zweite Plättchen wieder in linear polarisiertes Licht überführt wird. Die Richtung der Polarisation hängt dabei von der Orientierung des zweiten Plättchens ab. Über eine Reihe von Spiegeln gelangt das Licht anschließend zum rückseitigen Eingang des Mikroskops, wo es durch eine Linse (L3) fokussiert und von einem dichroitischen Spiegel (XF2045, Omega) auf die Rückapertur des Objektives reflektiert wird. Der Fokus liegt dabei auf Höhe der Rückapertur, damit der Bildausschnitt homogen im Weitfeld beleuchtet wird. Als Objektiv wird ein Öl-Immersions-Objektiv (UPlanSApo-100x-1.4, Olympus) verwendet, das wenig Einfluss auf die Qualität der Polarisation hat.

Von der Probe emittiertes Fluoreszenzlicht wird von dem Objektiv gesammelt und passiert anschließend den dichroitischen Spiegel. Dahinter ist ein Bandpassfilter (BP; 620/60 ET, AHF) mit einem Transmissionsbereich von $590 \mathrm{~nm}$ bis $650 \mathrm{~nm}$ installiert. Über ein Umlenkprisma wird das Emissionslicht aus dem seitlichen Ausgang des Mikroskops in ein lichtdichtes Gehäuse geführt. Darin befindet sich ein System aus zwei achromatischen Linsen (L6, L5), die das primäre Bild nachvergrößern und auf eine emCCD-Kamera (iXon 897, Andor) abbilden. Dabei wird die Vergrößerung von zunächst 100-fach auf 320-fach erhöht.

\subsubsection{Synchronisation von Modulation und Detektion}

Ein ausschlaggebender Faktor für die Qualität der gemessenen Daten ist eine korrekte Synchronisation der Bildfrequenz der Kamera mit der Rotation des linear polarisierten Anregungslichts. Die Frequenz der Kamera muss immer einem vielfachen der Rotationsfrequenz entsprechen. Eine variable Phasendifferenz, macht vor allem Datensätze unbrauchbar die zeitlich gemittelt werden müssen. Die verwendete emCCD-Kamera wird für alle Messungen im sogenannten Frame-Transfer-Modus betrieben. Dabei wird die nach der Belichtung erhaltene Ladung (bzw. das Bild) auf dem Chip in einen abgedunkelten Bereich verschoben und dort ausgelesen, während parallel die nächste Belichtung stattfindet. Bedingt durch diese Architektur ist es nicht möglich, die Belichtung durch ein externes Signal zu steuern. Aus diesem Grund wird die Rotation auf das konstante Kamerasignal synchronisiert. Hierfür wird der Motor eines optischen Chopper-Systems (Thorlabs, MC2000) verwendet, der über einen Zahnriemen die Rotation der Verzögerungsplatte antreibt. Die Rotationsgeschwindigkeit wird dabei durch eine an dem Motor angebrachte Lochscheibe, mithilfe einer Lichtschranke gemessen. Ein Regelkreis passt die Geschwindigkeit des Motors kontinuierlich an, um die Synchronisation mit dem Kamerasignal zu gewährleisten. 


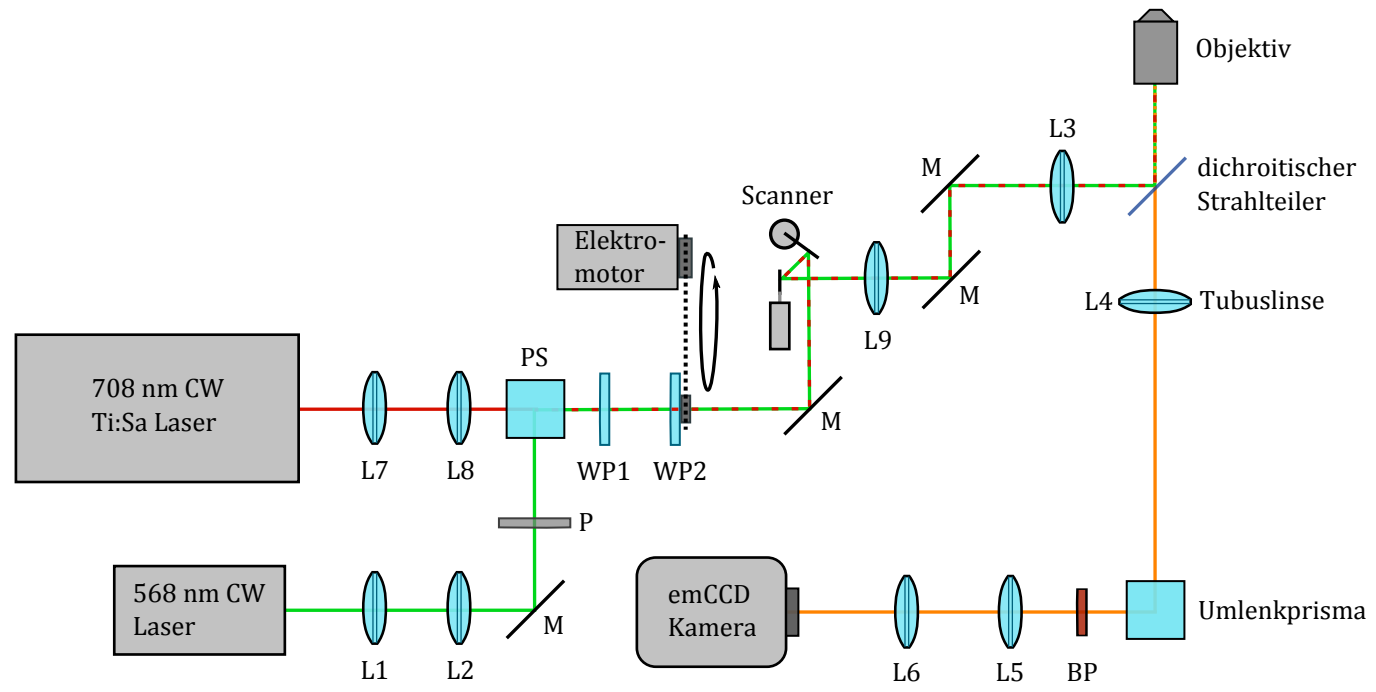

Abbildung 1.8: Illustration des optischen Aufbaus für ExPAN. M Spiegel, L Linse, PS polarisierender Strahlteilerwürfel, WP lambda-viertel Plättchen, P Polarisationsfilter, BP Bandpassfilter.

\subsubsection{Erweiterung durch ExPAN}

Der beschriebene experimentelle Aufbau wurde durch einen zweiten Laser erweitert, sodass der Winkelbereich der Anregung mittels stimulierter Emission verringert werden kann (siehe Abbildung 1.8). Die benötigte Leistungsdichte für die stimulierte Emission ist weitaus größere als die der Anregung. Aus diesem Grund wird hier ein leistungsstarker Titan:Saphir-Laser (Mira 900-F, Coherent) verwendet (siehe Abbildung 1.9), der durch einen frequenzverdoppelten Neodym:Vanadat-Laser (Verdi 18, Coherent) gepumpt wird. Der Titan:Saphir-Laser wird im Continuous Wave Modus betrieben und emittiert Licht mit einer Wellenlänge von $708 \mathrm{~nm}$. Der ausgekoppelte Laserstrahl passiert zunächst einen Kantenfilter (FEL700, Thorlabs), um kurzwelliges Pumplicht zu unterdrücken, das sonst zu einem Hintergrundsignal auf der Kamera beitragen würde. Anschließend wird der Strahl durch ein Linsenpaar aufgeweitet (L7, L8) und passiert einen elektronischen Verschlussmechanismus, bevor er über einen Strahlteilerwürfel in den Anregungsstrahlengang eingekoppelt wird (PB). Das Licht besitzt dabei eine zum Anregungslicht orthogonale Polarisation. Die Leistung des Lasersystems ist nicht ausreichend, um im gesamten Bildfeld $\left(\sim 25 \mu^{2}\right)$ stimulierte Emission zu induzieren. Aus diesem Grund wird der beleuchtete Bereich bei ExPAN-Experimenten verkleinert und ein 2-Achsen-Scanner verwendet, um den Bereich der Anregung über den kompletten Bildausschnitt zu bewegen. Auf diesem Wege wird eine höhere Leistungsdichte pro Flächeneinheit erreicht. Hierfür wird eine zusätzliche Linse hinter dem Scanner installiert (L9), um die Position des Fokus relativ zum Objektiv, und somit die Größe der ausgestrahlten Fläche, zu verändern. Bei dem Scanner handelt es sich um einen selbst konstruiertes Off-Axis-System, 


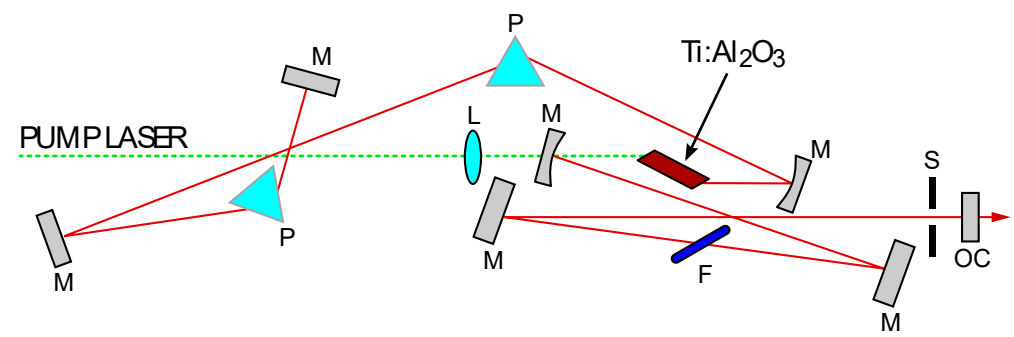

Abbildung 1.9: Strahlengang des Mira 900 Lasers: M Spiegel, P Prisma, L PumpstrahlFokuslinse, F doppelbrechender Filter, S Schlitzblende, OC Auskopplungsspiegel.

bestehent aus einem $16 \mathrm{kHz}$ resonannten Scanner (Electro-Optical Products Corp., SC-30) und einem Galvoscanner (M3, GSI Lumonics) für den Zeilenvorschub. Der Galvoscanner wird von einem DA-Wandler (USB-1608G-2AO, Measurement Computing) mit einer selbst erstellten Software gesteuert, die den Galvoscanner mit den resonanten Scanner und der Kamera synchronisiert.

\subsubsection{Proben}

\subsubsection{Präparation von Einzelmolekülproben}

Verwendete Deckgläschen werden zunächst gründlich mit Isopropanol und $\mathrm{ddH}_{2} \mathrm{O}$ gereinigt. Anschließend wird ein Tropfen $(30 \mu \mathrm{L})$ eine Lösung des Farbstoffs Atto 590 (50 nM freie Carbonsäure in Methanol, Atto-Tec) auf ein Deckgläschen gegeben. Nach Abdampfen des Lösungsmittels wird die Oberfläche mit einem Tropfen $\mathrm{ddH}_{2} \mathrm{O}$ benetzt und die Probe umgehend vermessen.

\subsubsection{Präparation von oberflächenmarkierten Microspheres}

Die Kammern eines LabTek-Objekträgers (Nuc Lab-Tek II, 8 Kammern, Sigma) wird für 10 min mit $400 \mu \mathrm{L}$ KOH-Lösung $(2 \mathrm{M})$ behandelt und anschließend 3-mal mit $400 \mu \mathrm{L}$ PBSPuffer (137 mM NaCl, $2.7 \mathrm{mM} \mathrm{KCl}, 10 \mathrm{mM} \mathrm{Na}_{2} \mathrm{HPO}_{4}, 1.8 \mathrm{mM} \mathrm{KH}_{2} \mathrm{PO}_{4}$ ) gewaschen. Jede Kammer wird mit $200 \mu \mathrm{L}$ einer BSA/BSA-Biotin-Lösung $(5 \mathrm{mg} / \mathrm{mL}$ Rinderserumalbumin, $0.5 \mathrm{mg} / \mathrm{mL}$ Biotin markiertes Rinderserumalbumin in PBS-Puffer, Sigma) befüllt und für mindestens 3 Stunden bei $7{ }^{\circ} \mathrm{C}$ inkubiert. Nach 4-maligem Waschen mit $400 \mu \mathrm{L}$ PBS-Puffer werden $400 \mu \mathrm{L}$ PBS-Puffer in jede Kammer gefüllt. $15 \mu \mathrm{L}$ einer verdünnten Suspension von Streptavidin-beschichteten Microspheres (10 $\mu \mathrm{g} \mathrm{mL}^{-1}$ in PBS, CP01N, Bangs Laboratories) mit einem mittleren Durchmesser von $0.51 \mu \mathrm{m}$ werden zugegeben und für 30 min inkubiert. Anschließend werden $20 \mathrm{\mu L}$ Atto 590 -biotin-Lösung ( $30 \mathrm{nM}$ in $\mathrm{ddH}_{2} \mathrm{O}$, Atto-Tec) in eine 
Kammer gegeben. Nach erreichen der gewünschten Farbstoffdichte wird die gesamte Lösung entfernt und durch $400 \mu \mathrm{L}$ PBS-Puffer ersetzt.

\subsubsection{Fixierte Hippocampus-Neuronen}

Präparate mit fixierten Hippocampus-Neuronen wurden freundlicherweise von Dr. Shih-Ju Lee $^{(4)}$ zur Verfügung gestellt. Primärkulturen von Ratten Hippocampi wurden hierfür gemäß dem Protokoll in [42] hergestellt und $\beta 3$-Tubulin mit dem Farbstoff Cy3 immunomarkiert. Die Proben wurden in Polyvinylalkohol (Mowiol 4-88, Sigma) fixiert.

\subsubsection{Algorithmus zur Auswertung von Einzelmolekül- daten}

Für die Auswertung von Einzelmoleküldaten wurde eine Bildrekonstruktionsalgorithmus verwendet, der von Dr. Timo Aspelmeier ${ }^{(5)}$ und Prof. Axel Munk ${ }^{(6)}$ entwickelt wurde. Der Algorithmus wird als sparsity penalty enhanced estimation by demodulation (kurz SPEED) bezeichnet und soll im Folgenden näher erläutert werden.

Wie in Abschnitt 1.2.4 beschrieben, weist jedes reale optische System physikalische Limitierungen und Abbildungsfehler auf, wodurch vor allem hohe Frequenzen in der Objektebene nur zu einem gewissen Grad in die Bildebene übertragen werden. Mithilfe der Methoden der Bildrekonstruktion kann versucht werden einen Teil dieser Informationen wiederherzustellen. Der Abbildungsprozess in einem optischen System lässt sich mathematisch durch ein Matrixprodukt beschreiben:

$$
\vec{b}=\mathbf{A} \vec{x}+\vec{e}
$$

Der Vektor $\vec{b}$ enthält das gemessene Bild, die Matrix A beschreibt die PSF des Abbildungssystems, $\vec{x}$ gibt die reale Dichte des gemessenen Objekts wieder und $\vec{e}$ ist der Messfehler, der z.B. durch Rauschen oder Quantisierungsfehler bei der Digitalisierung auftritt. Ziel ist es nun aus $\vec{b}$ und $\mathbf{A}$ das scharfe Bild $\vec{x}$ zu berechnen. Eine direkter Lösungsansatz ist die Berechnung von $\vec{x}=\mathbf{A}^{-\mathbf{1}}(\vec{b}-\vec{e})$, jedoch ist $\vec{e}$ im Normalfall nicht bekannt. Die Lösung der Gleichung unter Vernachlässigung des Fehlers in $\vec{b}$ führt jedoch zu unbrauchbaren Ergebnissen. Durch die PSF werden hohe Bildfrequenzen gedämpft oder gehen vollständig verloren, folglich nimmt das Signal-Rausch-Verhältnis mit zunehmender Frequenz ab und es kommt zu einem numerisch instabilen Gleichungssystem. Infolgedessen ist das resultierende Bild

\footnotetext{
(4) AG Stegmüller, Max-Planck-Institut für Experimentelle Medizin

(5) Institut für Mathematische Stochastik Göttingen

${ }^{(6)}$ Max-Planck-Institute für biophysikalische Chemie
} 
$\vec{x}$ mit einem hochfrequenten Rauschen überlagert und entspricht keiner sinnvollen Rekonstruktion [43, 44]. Gebräuchliche Algorithmen zur iterativen Dekonvolution arbeiten aus diesem Grund mit Regularisierungstechniken, wie z.B. der Total Variation Regularization [45], um unrealistische Lösungen zu unterdrücken. Ohne weitere Informationen lässt sich die Auflösung von mikroskopischen Aufnahmen damit in der Regel jedoch nicht vergrößern.

Die hier vorgestellte SPEED-Rekonstruktion versucht die zusätzliche Information aus dem modulierten Fluoreszenzsignal zu nutzen, um die angesprochenen Limitierungen zu umgehen und eine Rekonstruktion mit verbesserter Auflösung zu erzielen. Ein Datensatz enthält für jeden Bildpunkt neben der Gesamtintensität, die Phasenverschiebung $\Delta \alpha$ und Amplitude $A$ der Modulation. Allerdings wird das Signal eines Emitters durch die PSF auf einen gewissen Bildbereich verteilt und ist ggf. mit anderen Signalen überlagert. Ziel des Algorithmus ist es, einen neuen Satz an Parametern $I, \Delta \alpha$ und $A$ für jeden Pixel zu finden, der nach Faltung mit der PSF die gemessenen Daten möglichst adäquat wieder gibt. Dazu wird eine statistische Analyse benutzt, die auf einem Modell des Abbildungsprozesses basiert und im Folgenden kurz beschrieben werden soll:

Die Fluoreszenzintensität von einem Emitter $i$ an der Position $\overrightarrow{r_{i}}$ zur Zeit $t$ wird durch die Funktion $k\left(\alpha(t)-\alpha_{i}\right)$ beschrieben. Diese ist Abhängig von der Winkeldifferenzen zwischen der Anregungspolarisation $\alpha(t)$ und der Orientierung des Übergangsdipolmoments $\alpha_{i}$. Die Intensität wird mit der Form der PSF $U(r)$ auf dem Detektor abgebildet, sodass an der Position $r$ im Mittel $U\left(\vec{r}-\overrightarrow{r_{i}}\right) \cdot k\left(\alpha(t)-a_{i}\right)$ Poisson-verteilte Photonen detektiert werden. Aus der Summe aller Emitter ergibt sich somit das gemessene Bild

$$
I(\vec{r}, t) \sim \text { Poisson }\left(I_{0} \sum U\left(\vec{r}-\vec{r}_{i}\right) \cdot k\left(a(t)-a_{i}\right)\right),
$$

wobei in dem Vorfaktor $I_{0}$ werden Detektionseffizienz und Anregungsintensität zusammengefasst werden. Da die Orientierung der Anregungspolarisation im Experiment mit konstanter Geschwindigkeit rotiert, ist $\alpha(t)$ direkt proportional zu der Zeit und $k\left(\alpha(t)-\alpha_{i}\right)$ kann durch die Funktion $f\left(t-t_{i}\right)$ ersetzt werden. Zum Zeitpunkt $t_{i}$ sind Anregungspolarisation und Übergangsdipolmoment in der Fokusebene parallel. Für den Fall normaler Modulation (ohne ExPAN) ist $f(t)$ eine periodische Funktion der Form:

$$
f(t)=\cos ^{2}\left(\frac{\pi t}{T}\right)
$$

$T$ ist die Periodendauer und entspricht der Zeit in der sich der Polarisator um $180^{\circ}$ dreht. Für die weitere Betrachtung ist es von Vorteil den Ausdruck 1.17 auf eine Dichte an Emittern $g(\vec{r}, t) \mathrm{zu}$ beziehen, für die

$$
g(\vec{r}, t)=\sum_{i} \delta\left(\vec{r}-\vec{r}_{i}\right) \cdot \delta\left(t-t_{i}\right)
$$


gilt. Wobei $\delta(t)$ die eindimensionale und $\delta(\vec{r})$ die zweidimensionale Dirac-Funktion ist. Damit lässt sich der Ausdruck 1.17 umformulieren zu:

$$
I(\vec{r}, t) \sim \text { Possion }\left(I_{0} \int_{0}^{T} \int U\left(\vec{r}-\vec{r}^{\prime}\right) f\left(t-t^{\prime}\right) g(\vec{r}, t) \mathrm{d}^{2} r^{\prime} \mathrm{d} t^{\prime}\right)
$$

Im Vergleich mit klassischen Problemen der Bildrekonstruktion fällt auf, dass die gesuchte Dichte $g$ durch die Modulation der Anregungspolarisation eine zusätzliche Zeitdimension hat. Ohne diese Modulation ist nur eine reduzierte Dichte $\hat{g}(\vec{r})=\int_{0}^{T} g(\vec{r}, t) d t$ zugänglich. Dieser Umstand kann ausgenutzt werden, indem die Paradigmen des sog. Compressed sensing angewendet werden. Compressed sensing ermöglicht es ein Signal aus einem scheinbar ungenügenden Datensatz wiederherzustellen, wenn es eine Darstellung des Signals gibt, bei der die meisten Einträge Null sind [46]. Eine entsprechende Matrix wird als „dünn besetzt“ oder engl. sparse bezeichnet. Die zusätzliche Dimension von $g(\vec{r}, t)$ erhöht dessen sparsity. Im diskreten Fall hat die reduzierte Dichte $\hat{g}(\vec{r})$ ein Verhältnis zwischen Null und von Null verschiedenen Einträgen von $P / N$, wenn $N$ die Anzahl der Emitter und $P$ die Anzahl der Pixel ist. Ist $S$ die Anzahl an Bildern je Modulationsperiode, so hat $g(\vec{r}, t)$ ein Verhältnis von $P S / N$, und ist folglich $S$-fach dünner besetzt. Grundgedanke des Compressed sensing ist es, die Menge an möglichen Lösungen des Rekonstruktionsproblems einzuschränken, indem dünn besetzte Lösungen bevorzugt werden. Ein direktes Maß für die Besetzung ist dabei die $\ell_{0}$-Norm, welche die Anzahl der von Null verschiedenen Einträge eines Vektors angibt. Allerdings ist die Laufzeit für einen Algorithmus mit $\ell_{0}$-Regularisierung nicht praktikabel (die $\ell_{0}$-Minimierung ist NP-Schwer) [47]. Es konnte jedoch gezeigt werden, dass die Verwendung der $\ell_{1}$-Norm (Summennorm), anstelle der $\ell_{0}$-Norm, für viele Probleme äquivalente Lösungen liefert [48].

Im experimentellen Aufbau kommt es neben der Modulation der Polarisation auch zu einer Modulation der Anregungsintensität. Entsprechend ist das polarisationsabhängige Signal mit einer globalen Modulation gleicher Frequenz und konstanter Phase überlagert. Dieser Effekt muss bei der Auswertung berücksichtigt werden, damit keine Artefakte entstehen. Dazu wird der Vorfaktor $I_{0}$ durch eine zeitabhängige periodische Funktion $I_{0}(t)$ ersetzt, welche die Intensitätsschwankungen genau wiedergibt.

Des weiteren muss ein konstanter Hintergrund berücksichtigt werden. Dieser stammt vor allem durch Emitter über, oder unterhalb der Fokusebene, die entsprechen unscharf abgebildet werden. Da dadurch sehr viele Emitter mit unterschiedlichen Orientierungen zu jedem Bildpunkt beitragen, ist das resultierende Signal nahezu konstant. Dieser nicht modulierte Hintergrund wird durch eine zeitunabhängige Funktion $b(\vec{r})$ beschrieben. Unter der Annahme, dass dieser Hintergrund hauptsächlich aus unscharf abgebildeten Objekten außerhalb der Fokusebene besteht, sollte $b(\vec{r})$ vor allem niedrige Ortsfrequenzen beinhalten. Diese 
Eigenschaft lässt sich bei der Rekonstruktion erreichen, indem eine dünne Besetzung der Darstellung von $b(\vec{r})$ im Frequenzraum gefordert wird, sodass sich $b(\vec{r})$ aus wenigen, niedrigen Frequenzen zusammensetzt und hohe Frequenzanteile unterdrückt werden. Dabei wird die Kosinustransformation $\tilde{b}(\vec{k})=\mathcal{C O S}\{b(\vec{r})\}$ von $b(\vec{r})$ anstelle der Fourier-Transformation verwendet, um Komplikationen durch komplexe Funktionswerte bei der Implementierung zu vermeiden. Insgesamt ergibt sich somit folgender Ausdruck als Modellbeschreibung für die gemessenen Daten:

$$
I(\vec{r}, t) \sim \operatorname{Possion}(\mu(\vec{r}, t))
$$

mit

$$
\mu(\vec{r}, t)=I_{0}(t)\left(\int_{0}^{T} \int U\left(\vec{r}-\vec{r}^{\prime}\right) f\left(t-t^{\prime}\right) g\left(\vec{r}^{\prime}, t^{\prime}\right) \mathrm{d}^{2} r^{\prime} \mathrm{d} t^{\prime}+b(\vec{r})\right)
$$

Eine Schätzung der Dichte $g(\vec{r}, t)$ und des Hintergrunds $b(\vec{r})$ wird durch die Maximierung der Likelihood-Funktion mit Regularisierung über die $\ell_{1}$-Normen von $g$ und $\tilde{b}$ gewonnen. Die Likelihood-Funktion der Poisson-Verteilung mit dem Erwartungswert $\mu$ und einem einzelnen Messwert von $x$ detektierten Photonen ist definiert als [49]:

$$
L(\mu, x)=\frac{\mu^{x}}{x !} e^{-\mu}
$$

Üblicherweise wird bei der Maximum-Likelihood-Methode der negative Logarithmus der $L i$ kelihood-Funktion minimiert, da dieser deutlich einfacher zu berechnen ist:

$$
l(\mu, x)=-\ln (L(\mu, x))=\mu-x \ln (\mu)+\ln (x !)
$$

Daraus ergibt sich für die Zielfunktion $F(g, b, I)$ der Optimierung:

$$
F(g, b, I)=\int_{0}^{T} \int l(\mu(\vec{r}, t), I(\vec{r}, t)) \mathrm{d}^{2} r \mathrm{~d} t+\lambda_{1}\|g\|_{1}+\lambda_{2}\|\tilde{b}\|_{1}
$$

Dabei sind $I(\vec{r}, t)$ die gemessene Intensität an der Position $\vec{r}$ zur Zeit $t$ und $\|g\|_{1}$ sowie $\|\tilde{b}\|_{1}$ die $\ell_{1}$-Normen der gesuchten Dichte beziehungsweise des Cosinus-transformierten Hintergrunds. $\lambda_{1}$ und $\lambda_{2}$ sind empirisch zu bestimmende Regularisierungsparameter, die von dem experimentellen Aufbau und den Messbedingungen abhängen. Bei den in der Arbeit gezeigten Auswertungen wurden die Parameter in Regel auf $\lambda_{1}=1$ und $\lambda_{2}=1$ gesetzt. Die Zielfunktion $F(g, b, I)$ besteht aus dem konvex differenzierbaren Term der LikelihoodFunktion der Possionverteilung und den konvex undifferenzierbar Termen der $\ell_{1}$-Normen. Die effiziente Optimierung solcher Funktionen ist mit bestimmten Varianten des Gradientenverfahrens möglich. Der in dieser Arbeit verwendete SPEED-Algorithmus benutzt die Fast Iterative Shrinkage-Thresholding-Methode von Beck und Teboulle [50].

Ausgehend von einer Basisimplementierung von Dr. Aspelmeier wurde der hier beschriebene SPEED-Algorithmus in eine Software zur Datenauswertung umgesetzt. 


\subsubsection{Simulationen}

Zur Validierung des Auswertungsalgorithmus wurden Simulationen von Fluoreszenzemittern mit verschiedenen Anordnungen erstellt. Das Intensitätsprofil der Emitter wird als zweidimensionale Gauß-Verteilung mit einer Halbwertsbreite von 5 Pixel modelliert, was annähernd der Auflösung ( 250 nm FWHM) und Pixelgröße (50 nm/Pixel) im Experiment entspricht. Die Gauß-Verteilung ist dabei eine relativ gute Näherung für die PSF des Versuchsaufbaus. Jeder Emitter besitzt einen Phasenwinkel, der die Orientierung des Übergangsdipolmoments in der Fokusebene repräsentiert. Analog zum Experiment wird eine periodische Rotation des Anregungslichts simuliert, indem die Intensität eines Moleküls mit einer $\cos ^{2}$ Abhängigkeit moduliert wird (bzw. für ExPan über die Formel 1.37).

Statistische Schwankungen bei Messungen mit Einzelmolekül-Sensitivität sind hauptsächlich auf die begrenzte Anzahl der detektierten Photonen zurückzuführen und lassen sich somit durch eine Possionverteilung beschrieben. Aus diesem Grund wird die Fluktuation der detektierten Fluoreszenzintensität durch eine Poission-verteilte Zufallsvariable modelliert. Des weiteren besitzt die verwendete emCCD-Kamera ein Hintergrundsignal, das sich vereinfacht durch eine Kombination aus Normal- und Gammaverteilung beschreiben lässt. Der Beitrag einer gammaverteilten Zufallsvariable $\Gamma$ begründet sich durch den multiplikativen Charakter der Signalverstärkung in einer emCCD-Kamera [51]. Die Intensität für einen Pixel an der Position $\vec{r}$ im Fall einer einfachen Modulation ohne ExPAN ergibt sich somit zu:

$$
I_{k}(\vec{r})=B+\sum_{i} \operatorname{Possion}\left(I_{0} \cos ^{2}\left(\pi \frac{k}{T}+\varphi_{i}\right) U\left(\vec{r}-\vec{r}_{i}\right)\right)
$$

mit

$$
B \sim \mathcal{N}\left(\mu_{B}, \sigma_{B}\right)+\Gamma\left(\kappa_{B}, \theta_{B}\right) \quad \text { und } \quad U(\vec{r})=\frac{1}{2 \pi \sigma_{p s f}^{2}} \exp \left(-\frac{\vec{r}^{2}}{2 \sigma_{p s f}^{2}}\right)
$$

Dabei sind:
$k \quad$ Index des Frames
$\varphi_{i} \quad$ Phasenwinkel des $i$-ten Moleküls
$\vec{r}_{i} \quad$ Position des $i$-ten Moleküls
$T=10 \quad$ Periode der Modulation in Frames
$I_{0}=350 \quad$ Mittlere Anzahl der detektierten Photonen pro Molekül und Frame
$\mu_{B}=1.5 \quad$ Mittelwert des Hintergrunds
$\sigma_{B}=0.15$ Standartabweichung des Hintergrunds
$\kappa_{B}=0.82$ Formparameter der Gamma-Verteilung
$\theta_{B}=0.92$ Skalierungsparameter der Gamma-Verteilung 
Die Parameter für Signal- und Hintergrundintensität sind so gewählt sind, dass die Simulation die experimentellen Daten möglichst genau wiedergibt. 


\subsection{Theoretische Beschreibung von ExPAN}

Wenn elektronisch angeregte Fluorophore mit Licht einer geeigneter Wellenlänge bestrahlt werden, können diese durch stimulierte Emission wieder in den Grundzustand zurückkehren (siehe Kapitel 1.2.2). Ist die Geschwindigkeitskonstante der stimulierten Emission ähnlich oder größer als die der spontanen Emission, sinkt die Wahrscheinlichkeit, dass ein angeregtes Molekül fluoresziert. Bei der STED-Mikroskopie wird dieser Effekt zur Verbesserung der Auflösung von Konfokalmikroskopen genutzt, indem der Bereich verkleinert wird, in dem Farbstoffe Fluoreszenz emittieren [52-54].

Bei der hier vorgestellten Methode wird stimulierte Emission verwendet, um den Effekt der Photoselektion zu verstärken. Wie in Kapitel 1.2.1 beschrieben, ist die Anregungswahrscheinlichkeit proportional zu $\cos ^{2}(\alpha)$, wobei $\alpha$ der Winkel zwischen dem Übergangsdipolmoment des Fluorophors und dem Polarisationsvektor des Anregungslichts ist. Das detektierte Fluoreszenzsignal ist proportional zu der Population angeregter Fluorophore und somit in gleicher Weise abhängig von dem Winkel $\alpha$ wie die Anregungswahrscheinlichkeit. Wie im folgenden gezeigt wird, lässt sich die Winkelverteilung der Fluoreszenzanregung verkleinern, indem eine zweite Lichtquelle verwendet wird, die stimulierte Emission induziert und eine zum Anregungslicht orthogonale Polarisation besitzt. Da die $\cos ^{2}$-Winkelabhängigkeit ebenfalls für die stimulierte Emission gilt, werden hierdurch vor allem Fluorophore abgeregt, deren Übergangsdipolmoment eine ähnliche Orientierung aufweist, wie die Polarisation der stimulierenden Lichtquelle. In Abbildung 1.10 ist die Änderung der Winkelverteilung durch den Einsatz einer zweiten, stimulierenden Lichtquelle illustriert. In der Abbildung ist die Polarisation der anregenden elektromagnetischen Welle parallel zur x-Achse und die der stimulierenden, parallel zu der y-Achse. Durch die stimulierte Emission wird die Verteilung in Richtung der y-Achse gestaucht, sodass die Wahrscheinlichkeit Fluoreszenz zu emittieren für einen Emitter mit einem Übergangsdipolmoment in y-Richtung kleiner wird. Das detektierte Fluoreszenzsignal hat dadurch eine höhere Selektivität bezüglich der Orientierung der Fluorophore. Dieser Effekt wird im folgenden als ExPAN (excitation polarisation angle narrowing) bezeichnet.

Um ExPAN analytisch zu Beschreiben ist es sinnvoll die Polarisationsrichtungen der anregenden und der stimulierenden Lichtquelle durch die kanonischen Einheitsvektoren

$$
\vec{E}_{e x}=\left(\begin{array}{l}
1 \\
0 \\
0
\end{array}\right) \text { bzw. } \vec{E}_{s e}=\left(\begin{array}{l}
0 \\
1 \\
0
\end{array}\right)
$$



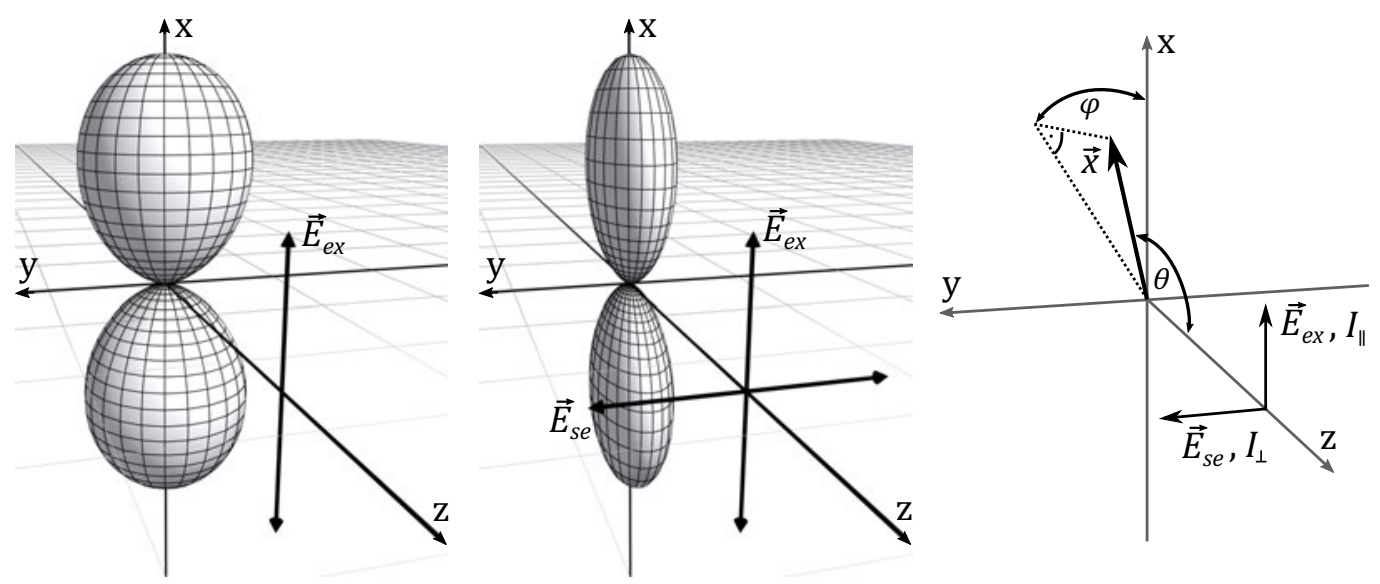

Abbildung 1.10: Auswirkung einer stimulierenden Lichtquelle mit orthogonaler Polarisation auf die Winkelverteilung angeregter Fluorophore. Links ohne und in der Mitte mit stimulierender Lichtquelle. Die Vektoren $\vec{E}_{e x}$ und $\vec{E}_{s e}$ zeigen die Polarisationsrichtung der anregenden- bzw. stimulierenden elektromagnetischen Welle an.

darzustellen. Die Orientierung des Übergangsdipolmoments kann durch einen Vektor auf der Einheitskugel beschrieben werden,

$$
\vec{x}(\theta, \varphi)=\left(\begin{array}{c}
\sin (\theta) \cos (\varphi) \\
\sin (\theta) \sin (\varphi) \\
1
\end{array}\right) .
$$

Dabei entsprechen die Winkel $\theta$ mit $0 \leq \theta \leq \pi$ und $\varphi$ mit $0 \leq \theta<2 \pi$ den Winkeln der Kugelkoordinaten (siehe Abbildung 1.10 rechts). Wie bereits erwähnt, ist die Fluoreszenzintensität proportional zu der Population der Fluorophore im angeregten Zustand. Ist das System im Gleichgewicht, lässt sich der Anteil der angeregten Fluorophore $N_{1}$ aus den Geschwindigkeitskonstanten aller relevanten Prozesse berechnen,

$$
N_{1}=\frac{k_{e x}}{k_{e x}+k_{f l}+k_{s e}} .
$$

Dabei sind $k_{e x}, k_{f l}$ und $k_{s e}$ die Geschwindigkeitskonstanten der Anregung, der Fluoreszenz und der stimulierten Emission. Die Wahrscheinlichkeiten für Anregung und der stimulierte Emission sind proportional zum Quadrat des Skalarprodukts von Polarisationsvektor und dem Übergangsdipolmoment (siehe Gleichung 1.5). Unter der Annahme, dass die Übergangsdipolmomente für beide Prozesse kollinear sind, gilt für die Geschwindigkeitskonstanten

$$
k_{e x}(\theta, \varphi)=k_{0, e x}\left(\vec{E}_{e x} \cdot \vec{x}(\theta, \varphi)\right)^{2}=k_{0, e x} \sin ^{2}(\theta) \cos ^{2}(\varphi)
$$

und

$$
k_{s e}(\theta, \varphi)=k_{0, s e}\left(\vec{E}_{s e} \cdot \vec{x}(\theta, \varphi)\right)^{2}=k_{0, s e} \sin ^{2}(\theta) \sin ^{2}(\varphi) .
$$


Abbildung 1.11: Orientierungsabhängige Fluoreszenzintensität für einen Emitter in der xy-Ebene mit ExPAN bei unterschiedlichen Werten für $f_{s}$.

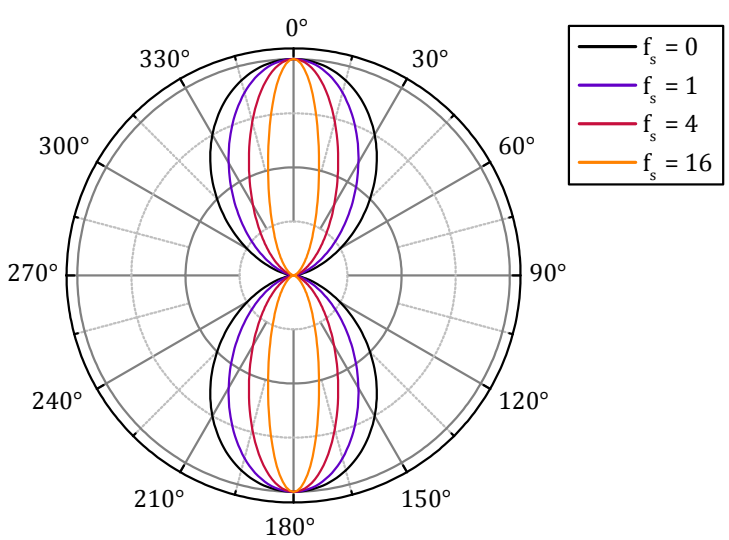

Hier sind $k_{0, e x}$ und $k_{0, s e}$ die maximalen Geschwindigkeitskonstanten bei $\vec{x}=\vec{E}_{e x}$ bzw. $\vec{x}=\vec{E}_{s e}$. Für Gleichung 1.30 ergibt sich somit:

$$
N_{1}=\frac{k_{0, e x} \sin ^{2}(\theta) \cos ^{2}(\varphi)}{k_{0, e x} \sin ^{2}(\theta) \cos ^{2}(\varphi)+k_{f l}+k_{0, s e} \sin ^{2}(\theta) \sin ^{2}(\varphi)}
$$

Bei kontinuierlicher Anregung mit für Weitfeldmikroskopie üblichen Intensitäten, ist die Geschwindigkeitskonstante der Anregung deutlich kleiner als die der Fluoreszenz, sodass Sättigungseffekte vernachlässigt werden können $\left(k_{f l} \gg k_{0, e x}\right)$. In diesem Fall lässt sich Gleichung 1.33 in guter Näherung zu

$$
N_{1} \approx \frac{k_{0, e x} \sin ^{2}(\theta) \cos ^{2}(\varphi)}{k_{f l}+k_{0, s e} \sin ^{2}(\theta) \sin ^{2}(\varphi)}
$$

vereinfachen. Des weiteren lässt sich ein Parameter für die Stärke des ExPAN-Effekts einführen, der die Geschwindigkeitskonstante der stimulierten, im Verhältnis zur spontanen Emission angibt, $f_{s}=k_{0, s e} / k_{f l}$ :

$$
N_{1} \approx \frac{k_{0, e x}}{k_{f l}} \frac{\sin ^{2}(\theta) \cos ^{2}(\varphi)}{1+f_{s} \sin ^{2}(\theta) \sin ^{2}(\varphi)}
$$

$f_{s}$ kann im Experiment durch die Intensität der stimulierenden Lichtquelle eingestellt werden. Die orientierungsabhängige Fluoreszenzintensität eines Emitters ist damit näherungsweise proportional zu

$$
I(\theta, \varphi) \propto \frac{\sin ^{2}(\theta) \cos ^{2}(\varphi)}{1+f_{s} \sin ^{2}(\theta) \sin ^{2}(\varphi)} .
$$

Liegt das Übergangsdipolmoment in der xy-Ebene (bzw. in der Fokusebene im Experiment) ist $\theta=\pi / 2$, wodurch sich Ausdruck 1.36 weiter vereinfacht $\mathrm{zu}$

$$
I(\varphi) \propto \frac{\cos ^{2}(\varphi)}{1+f_{s} \sin ^{2}(\varphi)} .
$$

In Abbildung 1.11 ist die Funktion 1.37 bei verschiedenen Werte für $f_{s}$ in einer Polardarstellung aufgetragen. Mit zunehmendem Werte für $f_{s}$ wird die Winkelverteilung schmaler. Das heißt, je größer die Intensität der stimulierenden Lichtquelle ist, desto selektiver wird die Fluoreszenzemission auf Fluorophore mit der entsprechenden Orientierung eingegrenzt. 


\subsubsection{Fluoreszenzanisotropie}

ExPAN hat ebenfalls Einfluss auf die Fluoreszenzanisotropie und ist damit von potentiellem Interesse für Methoden die auf der Messung der Fluoreszenzdepolarisation beruhen [55]. Aus diesem Grund soll dieser Effekt hier kurz theoretisch betrachtet werden.

Die Fluoreszenzanisotropie $r$ ist definiert als [34]

$$
r=\frac{I_{\|}-I_{\perp}}{I_{\|}+2 I_{\perp}} .
$$

$I_{\|}$und $I_{\perp}$ sind die Fluoreszenzintensitäten, gemessen durch einen Polarisationsfilter mit paralleler bzw. orthogonaler Ausrichtung bezogen auf die Anregungspolarisation. Für gewöhnlich ist der Detektor bei einem Anisotropie-Experiment im rechten Winkel zum Anregungsstrahl ausgerichtet, in diesem Fall wird die Fluoreszenzintensität jedoch parallel zum Anregungsstrahlengang bzw. der z-Achse detektiert (siehe Abbildung 1.10 rechts). Somit wird $I_{\|}$parallel zu $\vec{E}_{e x}$ und $I_{\perp}$ parallel zu $\vec{E}_{s e}$ detektiert. Für einen einzelnen Emitter mit einem Übergangsdipolmoment in Richtung des Vektors $\vec{x}(\theta, \varphi)$ ist die detektierte Intensität $I_{\|}$proportional zu $\left(\vec{E}_{e x} \cdot \vec{x}(\theta, \varphi)\right)^{2}$ und $I_{\perp}$ proportional zu $\left(\vec{E}_{s e} \cdot \vec{x}(\theta, \varphi)\right)^{2}$. Unter Berücksichtigung der Photoselektion durch Anregung und stimulierter Emission nach Gleichung 1.36, ergeben sich die gemessenen Intensitäten für die beiden Orientierungen der Polarisationsfilter $\mathrm{zu}$

und

$$
I_{\|}(\theta, \varphi)=\left(\vec{E}_{e x} \cdot \vec{x}(\theta, \varphi)\right)^{2} I(\theta, \varphi)=\frac{\sin ^{4}(\theta) \cos ^{4}(\varphi)}{1+f_{s} \sin ^{2}(\theta) \sin ^{2}(\varphi)}
$$

$$
I_{\perp}(\theta, \varphi)=\left(\vec{E}_{s e} \cdot \vec{x}(\theta, \varphi)\right)^{2} I(\theta, \varphi)=\frac{\sin ^{4}(\theta) \sin ^{2}(\varphi) \cos ^{2}(\varphi)}{1+f_{s} \sin ^{2}(\theta) \sin ^{2}(\varphi)} .
$$

Die Intensität für ein Ensemble von Emittern mit statistisch verteilter Orientierung lässt sich durch Integration über die Kugeloberfläche berechnen:

$$
\begin{aligned}
I & =\int I(\theta, \varphi) \mathrm{d} A=\int_{0}^{2 \pi} \int_{0}^{\pi} I(\theta, \varphi) \sin (\theta) \mathrm{d} \theta \mathrm{d} \varphi \\
I_{\|}\left(f_{s}\right) & =\int_{0}^{2 \pi} \int_{0}^{\pi} \frac{\sin ^{5}(\theta) \cos ^{4}(\varphi)}{1+f_{s} \sin ^{2}(\theta) \sin ^{2}(\varphi)} \mathrm{d} \theta \mathrm{d} \varphi \\
I_{\perp}\left(f_{s}\right) & =\int_{0}^{2 \pi} \int_{0}^{\pi} \frac{\sin ^{5}(\theta) \sin ^{2}(\varphi) \cos ^{2}(\varphi)}{1+f_{s} \sin ^{2}(\theta) \sin ^{2}(\varphi)} \mathrm{d} \theta \mathrm{d} \varphi
\end{aligned}
$$

Die Integrale in Ausdruck 1.43 und 1.42 sind numerisch lösbar. Mit Gleichung 1.36 ergibt sich

$$
r_{0}\left(f_{s}\right)=\frac{I_{\|}\left(f_{s}\right)-I_{\perp}\left(f_{s}\right)}{I_{\|}\left(f_{s}\right)+2 I_{\perp}\left(f_{s}\right)},
$$

wobei $r_{0}$ hier die maximale Fluoreszenzanisotropie ist. Dieser Wert ist nur zu beobachten, wenn Absoptions- und Emissionsübergangsdipolmomente kollinear sind und keine weiteren 
Abbildung 1.12: Maximale Fluoreszenzanisotropie aufgetragen gegen die Intensität von ExPAN durch numerische Integration von Gleichung 1.44 .

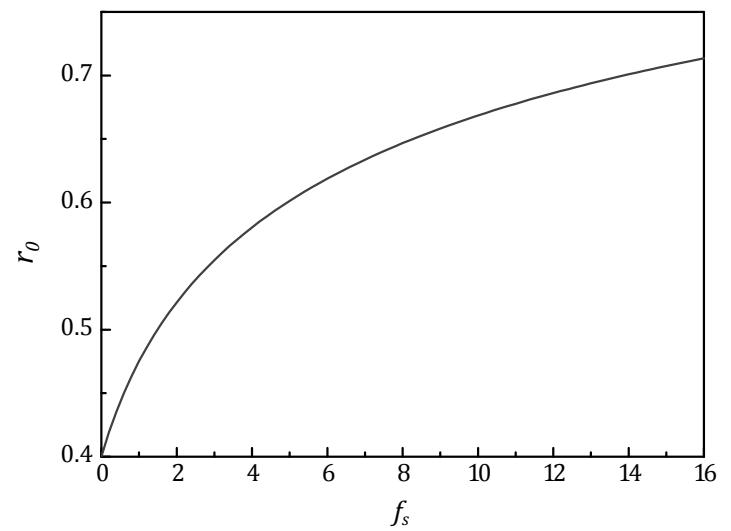

Prozesse zur Depolarisation beitragen. Durch Einphotonenanregung kann die Fluoreszenzanisotropie grundsätzlich nicht größer als $2 / 5=0.4$ werden [34]. Eine größere Anisotropie ist im Normalfall nur durch nichtlineare Anregung möglich. Durch Zwei-Photonen-Absorption kann so ein $r_{0}$ von $4 / 7 \approx 0.57$ erreicht werden [56]. Wie die Auftragung in Abbildung 1.12 zeigt, lässt sich mit ExPAN jedoch eine deutlich größere Fluoreszenzanisotropie erreichen. Mit zunehmender Intensität für die stimulierende Lichtquelle steigt auch $r_{0}$. Dieser Effekt tritt allerdings nur in Richtung der z-Achse auf. Die maximale Anisotropie entlang der yAchse (siehe Abbildung 1.10 rechts) bleibt auf einen Wert von 0.4 beschränkt.

Bei zeitaufgelösten Fluoreszenzdepolarisations-Experimenten wird eine Probe mit einer gepulsten Lichtquelle angeregt und der zeitliche Abfall der Fluoreszenzanisotropie erfasst, der in der Regel durch Rotationsdiffusion verursacht wird. Die Amplitude der Anisotropieabklingkurve wird dabei durch die maximale Anisotropie begrenzt. Theoretisch könnte mit ExPAN bei solchen Experimenten eine deutlich höhere Anfangspolarisation erreicht werden, wodurch sich der Dynamikumfang der Messung signifikant steigern ließe.

Für viele Fluoreszenzfarbstoffe sind die Übergangsdipolmomente der Emission und Absorption nicht exakt kollinear. Abhängig vom verwendeten Farbstoff kann $r_{0}$ bei konventionellen Anisotropie-Experimenten dadurch deutlich kleiner als 0.4 sein [57]. Eine interessante Eigenschaft von ExPAN ist, dass eine hohe Fluoreszenzpolarisation generell auch bei Fluorophoren erreicht werden kann, bei denen die Übergangsdipolmomente für Emission und Absorption deutlich voneinander abweichen, denn die Effektivität der Fluoreszenzlöschung durch die stimulierende Lichtquelle ist nur von der Orientierung des Übergangsdipolmoments der Emission abhängig. 


\subsection{Ergebnisse und Diskussion}

\subsubsection{Messungen von Einzelmolekülen}

Für Experimente an Einzelmolekülen auf einer Glasoberfläche wurde der Fluoreszenz-Farbstoff Atto 590 verwendet. Atto 590 besitzt ein Absorptionsmaximum bei $594 \mathrm{~nm}$ und ist damit kompatibel zu der verwendeten Anregungslichtquelle mit einer Wellenlänge von $568 \mathrm{~nm}$. Des weiteren zeichnet sich der Farbstoff durch eine hohe Photostabilität aus und eignet sich somit für Einzelmolekül-Experimente sowie für die Fluoreszenzlöschung durch stimulierte Emission [58]. Atto 590 gehört zu der Gruppe der Rhodamin-Farbstoffe, die auf der Xanthen Struktur basieren. Das Übergangsdipolmoment für die Absorption in den ersten angeregten Zustand liegt bei Xanthen-Derivaten typischerweise entlang der langen Achse der Xanthen-Einheit (siehe Abbildung 1.13) [59,

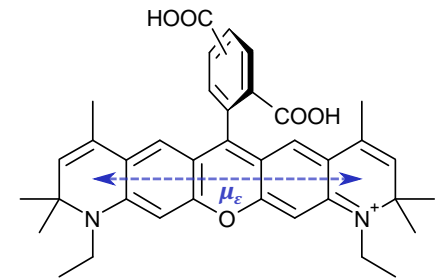

Abbildung 1.13: Strukturformel des Fluoreszenzfarbstoffs Atto 590 als freie Carbonsäure. Der blaue Pfeil illustriert die Orientierung des Übergangsdipolmoments für den Übergang $\mathrm{S}_{0} \rightarrow \mathrm{S}_{1}$. $60]$.

In Abbildung 1.14 ist eine Messung von vereinzelten Atto 590 Molekülen auf einem Glassubstrat dargestellt. Für das Bild wurden 80 Einzelbilder mit einer Belichtungszeit von jeweils $30 \mathrm{~ms}$ addiert, wobei die Anregungspolarisation während der Messung kontinuierlich rotiert wurde. In einer Rotationsperiode wurden dabei 10 Einzelbilder aufgenommen. Die Summe der Einzelbilder entspricht einer Messung mit unpolarisierter Anregung. Abbildung 1.15 zeigt den markierten Ausschnitt bei den 10 verschiedenen Orientierungen der Anregungspolarisation. Hier ist zu erkennen, dass ist die Fluoreszenzintensität einzelner Moleküle stark von der Ausrichtung der Anregungspolarisation abhängig ist. Folglich ist die Wechselwirkung zwischen Glasoberfläche und Farbstoffmolekülen ausreichend, um eine Rotationsbewegung der Moleküle zu unterbinden. Da das Signal im Intensitätsminimum fast vollständig verschwindet, muss das Übergangsdipolmoment zudem nahezu parallel zur Oberfläche ausgerichtet sein.

Aufgrund der kontinuierlichen Rotation der Anregungspolarisation, lässt sich die gemessene Intensität als zeitabhängiges Signal auffassen. In Abbildung 1.16 ist der entsprechende Signalverlauf eines einzelnen Moleküls (markiert in Abbildung 1.15) repräsentativ aufgetragen. Die Anpassung eines Sinusoides an das periodische Signal zeigt eine gute Übereinstimmung 
mit der erwarteten $\cos ^{2}$-Abhängigkeit ${ }^{(7)}$. Der Phasenwinkel der angepassten Funktion steht hierbei im direkten Zusammenhang mit der Ausrichtung des Moleküls in der Fokusebene.

Eine Parameteranpassung in jedem Bildpunkt ist bei Daten mit geringem Signal-RauschVerhältnis wenig robust, daher ist es sinnvoller eine Fourier-Transformation zu verwenden. Dadurch lässt sich die Phase und die Amplitude in jedem Bildpunkt direkt aus dem FourierKoeffizienten für die Modulationsfrequenz gewinnen. In Abbildung 1.17 ist eine entsprechende Auswertung der Daten aus Abbildung 1.14 illustriert. Im oberen linken Bild ist die Größe der Amplitude dargestellt, die dem gemittelten Bild aus Abbildung 1.14 stark ähnelt. Eine genauere Betrachtung zeigt allerdings, dass das Hintergrundrauschen im Amplitudenbild deutlich kleiner ist, als bei den gemittelten Daten. Da nur die Frequenzkomponente der Polarisationsmodulation zu der Amplitude beiträgt, werden konstante Signalanteile hier nicht berücksichtigt und Rauschanteile, die sich über das gesamte Frequenzspektrum verteilen, deutlich verringert. Im unteren linken Bild in Abbildung 1.17 ist die zugehörige Phase abgebildet. Diese lässt sich nur für Bildbereiche mit ausreichend Signal interpretieren. Wird die Phase als Farbton und die Amplitude als Helligkeit kodiert, lässt sich ein einfacher zu überschauendes Falschfarben-Bild erzeugen (Abbildung 1.17 rechts). Der Phasenwinkel ein-

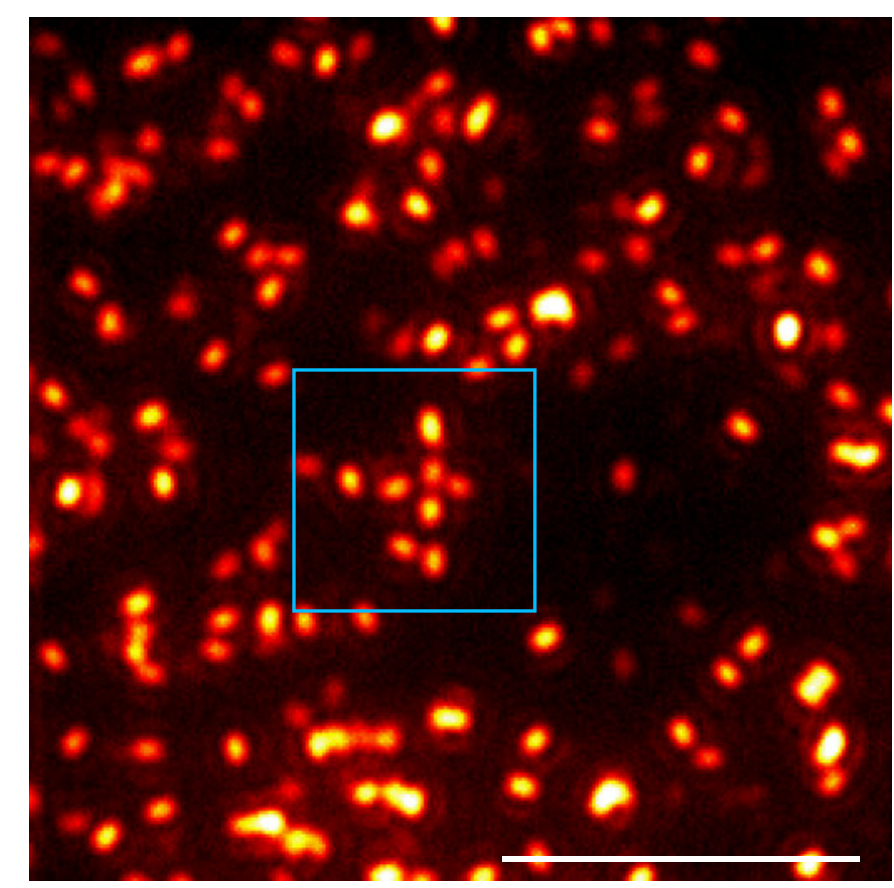

Abbildung 1.14: Aufnahme von vereinzelten Atto 590 Moleküle auf einem Glassubstrat aus der Mittlung von 80 Einzelbildern. (Maßstab: $5 \mu \mathrm{m}$ )

(7) Dabei gilt $\cos ^{2}(x)=\frac{1}{2}+\frac{1}{2} \sin \left(2 x-\frac{\pi}{2}\right)$. 

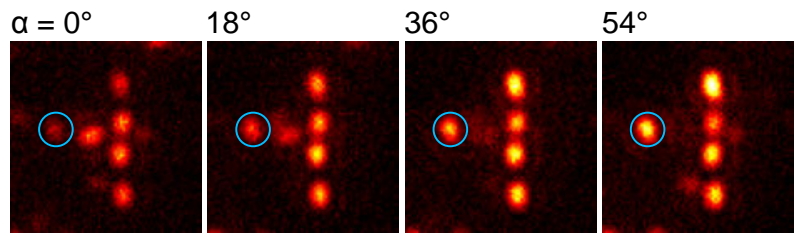

$72^{\circ}$

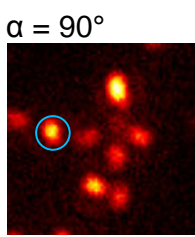

$108^{\circ}$

$126^{\circ}$

$144^{\circ}$
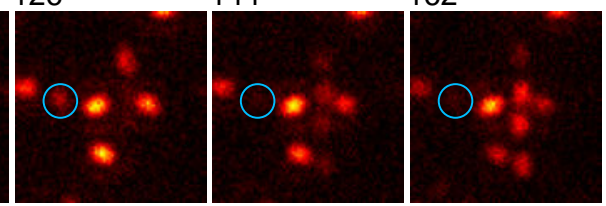

Abbildung 1.15: Ausschnitt des in Abbildung 1.15 markierten Bereichs bei verschieden Orientierungen der Anregungspolarisation.

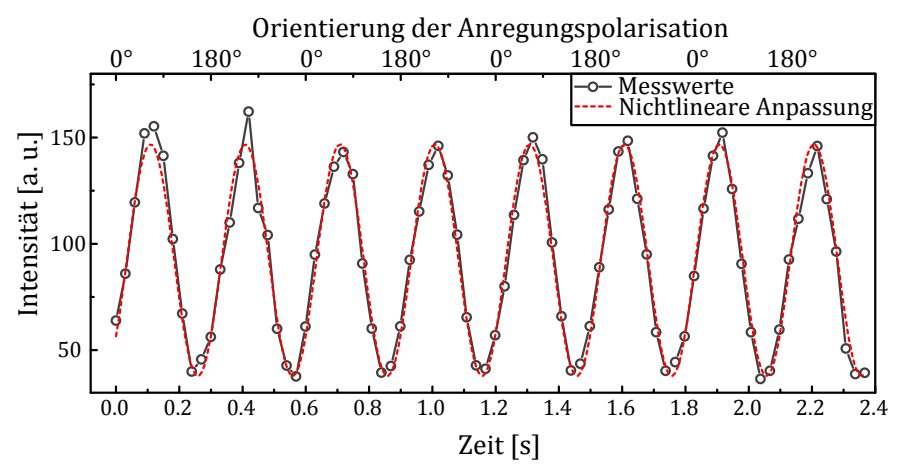

Abbildung 1.16: Mittlere Intensität der in Abbildung 1.15 blau umrandeten Fläche bei kontinuierlich rotierter Anregungspolarisation. In rot ist die sinusoide Anpassung der Form $I(t)=I_{0} \sin (\omega t+\varphi)+C$ mit $\omega=2 \pi \cdot 3.3 \mathrm{~Hz}$ aufgetragen.

zelner Signale kann direkt aus der Abbildung ablesen werden und deutet erwartungsgemäß auf eine zufällige Orientierung der Moleküle hin.

Die Intensitätsprofile einzelner Moleküle aus der Messung in Abbildung 1.16 weisen eine leichte Asymmetrie auf. Die Ursache hierfür ist eine geringfügige Defokussierung der Probe. Aufgrund der Abstrahlungseigenschaften eines elektrischen Dipols entsteht dadurch eine charakteristische Intensitätsverteilung, die von der Dipol-Orientierung abhängig ist. Dieser Effekt eröffnet die Möglichkeit, die aus der Phase gewonnene Ausrichtung der Emitter mit einer gesonderten Messgröße zu verifizieren. Die exakte Beschreibung der Intensitätsverteilung ist komplex und hängt von der genauen dreidimensionalen Orientierung des Dipols sowie dem Ausmaß der Defokussierung ab [26]. In diesem Fall ist allerdings nur die Orientierung in der Fokusebene, also der Azimutwinkel des Moleküls, von Interesse. Aufgrund der nur geringfügigen Defokussierung ist eine Anpassung mit einer asymmetrischen 2D-GaußFunktion zur Bestimmung des Azimutwinkels ausreichend. In Abbildung 1.18 (rechts) ist das Ergebnis einer entsprechenden Anpassung illustriert. Die rote und grüne Linie markieren die 

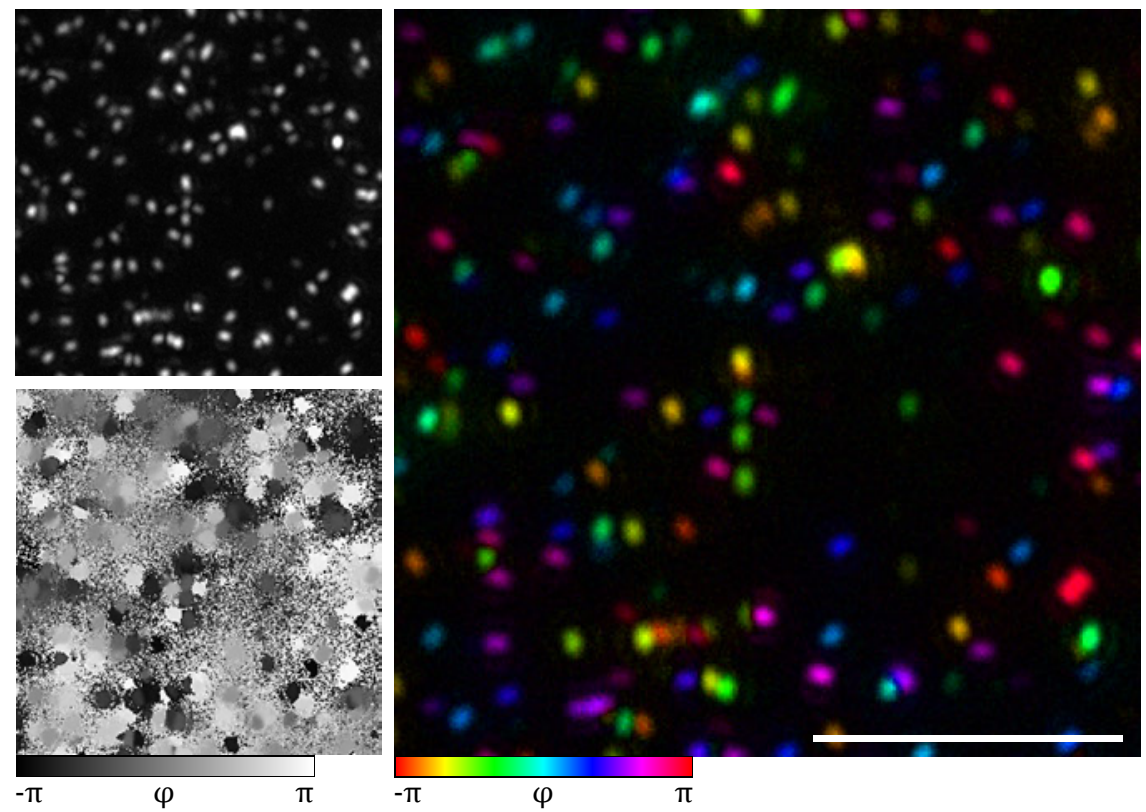

Abbildung 1.17: Visualisierung der Fourier-Koeffizienten. Links oben: Amplitude. Links unten: Phasenwinkel. Rechts: Phase als Farbton und Amplitude als Helligkeit. (Maßstab: 5 um)
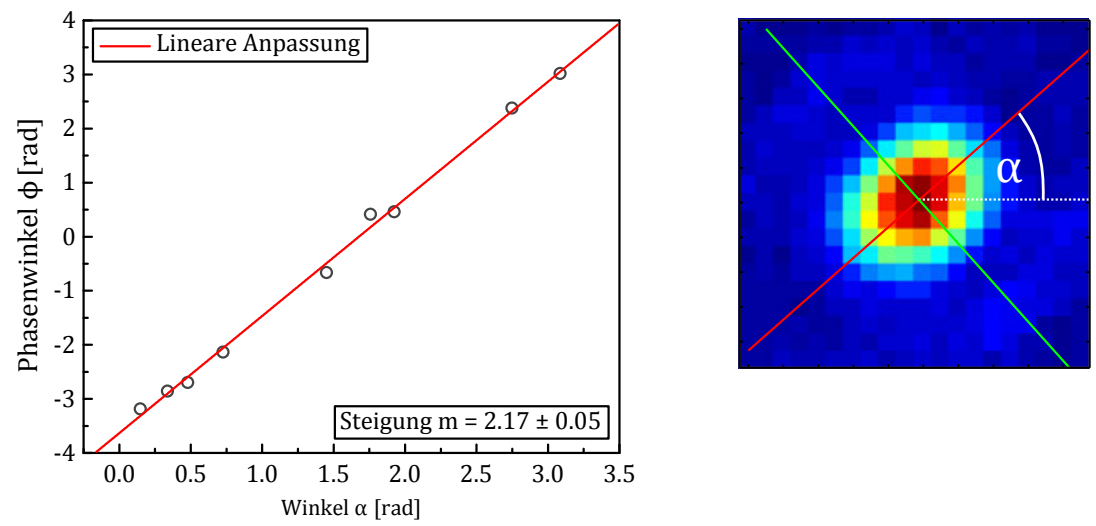

Abbildung 1.18: Links: Phasenwinkel aus den Fourier-Koeffizienten für die Signale einzelner Moleküle aufgetragen gegen die Orientierung einer 2D-Gauß-Anpassung. Rechts: Fluoreszenz eines einzelnen Moleküls und die aus der Gauß-Anpassung gewonnen Orientierung.

kurze bzw. lange Achse der angepassten Funktion. Der aus der Anpassung gewonnene Winkel $\alpha$ entspricht dem Azimutwinkel des Übergangsdipolmoments. Auf der linken Seite sind die Ergebnisse der Anpassung sowie die aus der Fourier-Trans gewonnen Phasenwinkel für eine für eine Reihe von Molekülen aufgetragen. Dabei wurden isolierte Moleküle aus dem Datensatz von Abbildung 1.17 ausgewählt und deren Phase durch eine Mittelung der korrespondierenden Bildpunkte im Phasenbild bestimmt. Die gleichen Signale wurden ebenfalls über eine Gauß-Anpassung ausgewertet. Die Auftragung zeigt eine gute Übereinstimmung 


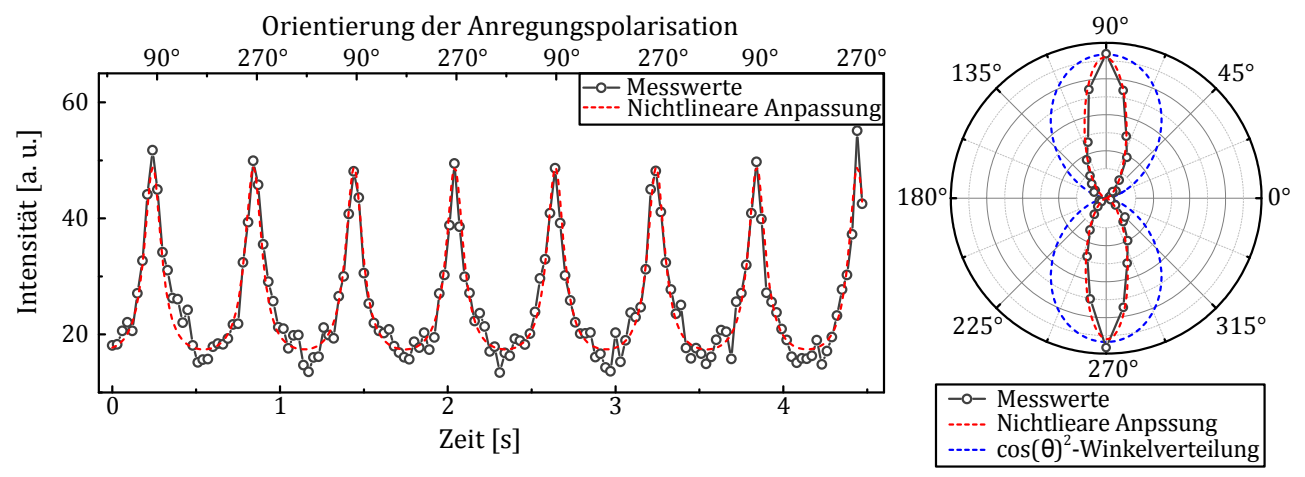

Abbildung 1.19: Oben: Fluoreszenzsignal eines einzelnen Atto 590 Moleküls bei unterschiedlichen Orientierungen der Anregungspolarisation mit ExPAN (ExPAN-Intensität $\sim 1.7 \mathrm{MW} \mathrm{cm}^{-2}$ ). Unten links: Auftragung des Signalverlaufs. Rechts: Darstellung der Daten in einem Polardiagramm zusammen mit einer $\cos ^{2}$-Verteilung. Für die nichtlineare Anpassung wurde die Funktion 1.37 verwendet.

der beiden Methoden, wobei zu berücksichtigen ist, dass der Phasenwinkel dem doppelten Azimutwinkel zuzüglich eines Offsets entspricht.

\subsubsection{Messungen von Einzelmolekülen mit ExPAN}

Aus der Summe zweier Sinusoide gleicher Frequenz resultiert wiederum ein Sinusoid ${ }^{(8)}$. Für einen Bildpunkt lässt sich dementsprechend nur eine Phase und eine Amplitude bestimmen, selbst wenn das gemessene Signal durch die Überlagerung von mehreren Emittern entstanden ist. Dadurch wird es mit zunehmender Dichte schwieriger die Signale einzelner Emitter zu separieren. Diese Limitierung lässt sich jedoch umgehen, indem die Form des modulierten Fluoreszenzsignals in geeigneter Weise verändert wird. Wie in Kapitel 1.4 theoretisch beschrieben, ist es prinzipiell möglich den Winkelbereich der Anregung durch stimulierter Emission zu verringern. Um dieses Konzept experimentell zu Überprüfen, wurde der Versuchsaufbau durch einen zweiten Laser für stimulierte Emission erweitert (siehe Kapitel 1.3.1.2). Die Abbildung 1.19 zeigt das Fluoreszenzsignal eines einzelnen Atto 590 Moleküls aus einer Messung mit ExPAN. In der Polardarstellung ist zu erkennen, dass die Winkelverteilung der Fluoreszenzintensität deutlich schmaler ist, als die $\cos ^{2}$-Verteilung. Im Experiment verteilt sich das Fluoreszenzsignal dadurch auf weniger Einzelbilder und die Maxima des Signals werden entsprechend spitzer. Eine nichtlineare Anpassung nach Gleichung 1.37 zeigt, dass die gemessene Kurve sehr gut mit der theoretisch zu erwartenden Winkelabhängigkeit übereinstimmt. Die durch den Parameter $f_{s}$ bestimmte Intensität des ExPAN-

\footnotetext{
${ }^{(8)}$ Für die Summe zweier Sinusoide gleicher Frequenz gilt: $A \sin (\omega t+\alpha)+B \sin (\omega t+\beta)=C \sin (\omega t+\gamma)$ mit $C=\sqrt{(A \cos (\alpha)+B \cos (\beta))^{2}+(A \sin (\alpha)+B \sin (\beta))^{2}}, \gamma=\tan ^{-1}\left(\frac{A \sin (\alpha)+B \sin (\beta)}{A \cos (\alpha)+B \cos (\beta)}\right)$
} 

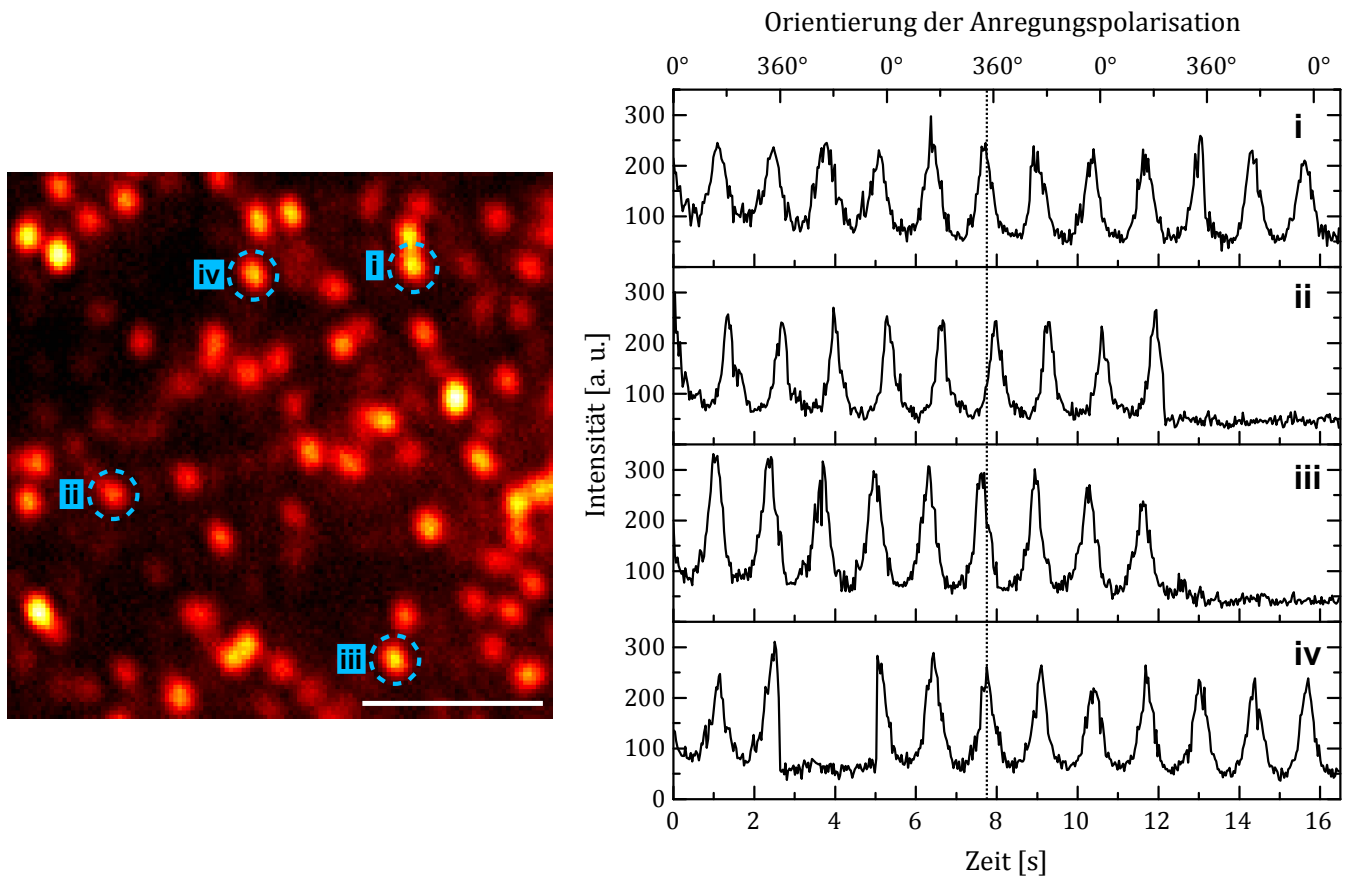

Abbildung 1.20: Links: Ausschnitt einer Messung von vereinzelten Atto 590 Molekülen mit ExPAN (Mittlung über 500 Einzelbildern, ExPAN-Laserintensität $\sim 1.7 \mathrm{MW} \mathrm{m}^{-2}$ ). Rechts: Intensitätsverlauf für die im Bild markierten Moleküle. Moleküle ii und iii werden während der Messung gebleicht, iv zeigt einen Übergang in einen reversiblen dunklen Zustand. (Maßstab: $2 \mu \mathrm{m})$

Effekts beträgt hier $f_{s}=9.3 \pm 0.8$. Dementsprechend ist die Geschwindigkeitskonstante der stimulierten Emission etwa um den Faktor 10 größer, als die der spontanen Emission.

Da die Größe des Effekts von der Intensität der stimulierenden Lichtquelle abhängig ist, lässt sich theoretisch ein beliebig schmales Intensitätsmaximum erreichen. In der Praxis ist die Ausgangsleistung des eingesetzten Lasersystems begrenzt, vor allem aber die Zunahme an Photobleichen limitiert die einsetzbare Leistung. Verglichen mit Messungen ohne ExPAN konnte schon bei der verwendeten Bestrahlungsstärke von $1.7 \mathrm{MW} \mathrm{cm}^{-2}$ ein signifikant schnelleres Photobleichen sowie vermehrt Übergänge in reversible dunkle Zustände beobachtet werden. In Abbildung 1.20 sind einige Intensitätsverläufe für Farbstoffmoleküle aufgetragen, die dieses Verhalten zeigen. Bei den ii und iii markierten Signalen ist ein permanentes Photobleichen zu erkenne. Das Molekül in iv geht hingegen für eine kurze Zeit in einen nicht-fluoreszenten Zustand über.

Relaxationspfade die zum Photobleichen führen gehen in den meisten Fällen von höher angeregte elektronischen Zustände aus, die durch Absorption aus dem $\mathrm{S}_{1}$-Zustand populiert werden können (Excited State Absorption) [61, 62]. Das verstärkte Photobleichen im Expe- 

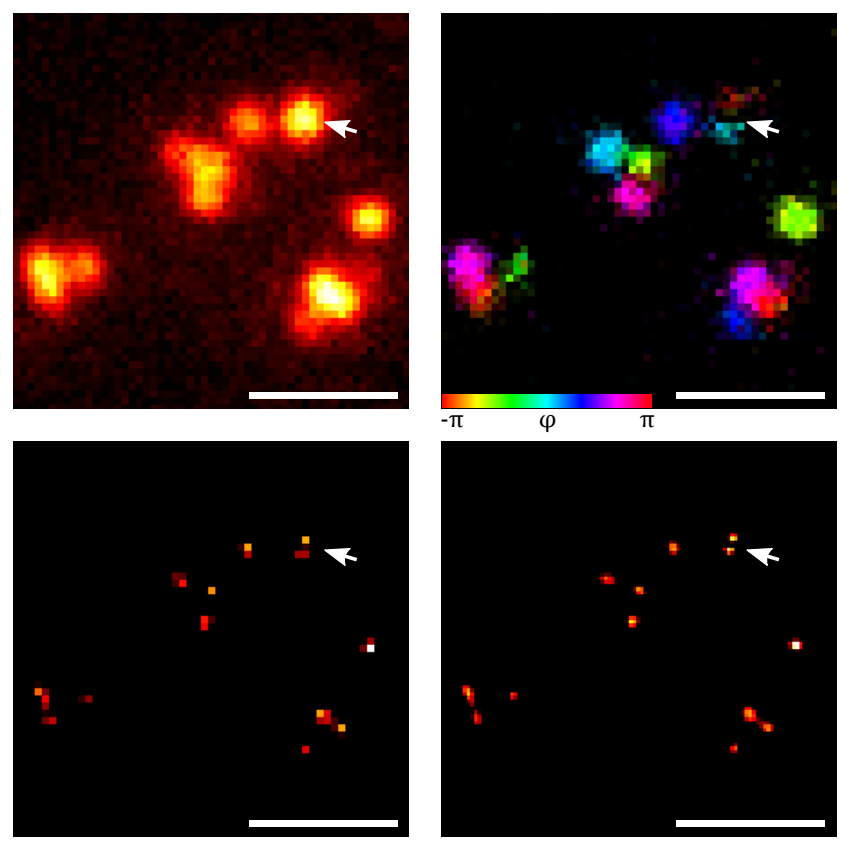

Abbildung 1.21: Auswertung einer Messung von einzelnen Atto 590 Molekülen mit dem SPEED-Algorithmus. Oben links: Summe über 60 Einzelbilder. Oben rechts: Visualisierung der Fourier-Koeffizienten. Unten links: SPEED-Auswertung $\left(\lambda_{1}=1, \lambda_{2}=1\right)$. Unten rechts: SPEEDAuswertung mit zweifachem Oversampling und den Parametern wie links. (Maßstab: $1 \mu \mathrm{m}$ )

riment lässt sich mit der Anregung solcher Übergänge durch die stimulierende Lichtquelle erklären.

\subsubsection{Auswertung mit dem SPEED-Algorithmus}

Die Abbildung 1.21 zeigt den Ausschnitt einer Messung von Atto 590 Molekülen sowie die entsprechende Auswertung mit dem SPEED-Algorithmus (siehe Kapitel 1.3.3). Im oberen linken Ausschnitt ist die Summe von 60 Einzelbildern (30 ms Belichtungszeit, 6 Perioden) der Messung abgebildet. Hier sind mehrere Farbstoffmoleküle zu erkennen, deren Signale sich aufgrund ihrer räumlichen Nähe überlagern. Die Visualisierung der Fourier-Koeffizienten (oben rechts) zeigt, dass den Signalen dennoch eine Phase zugeordnet werden kann. Zum Beispiel lässt sich bei dem markierten Signal (Pfeil) erkennen, dass dieses aus der Überlagerung zweier Moleküle mit annähernd senkrechter Orientierung entstanden ist, wodurch sich die Modulation im Zentrum nahezu aufhebt.

Der Ausschnitt unten links (Abbildung 1.21) zeigt das Ergebnis der Rekonstruktion durch den SPEED-Algorithmus. Der Ursprung der einzelnen Signale konnte durch die Auswer- 


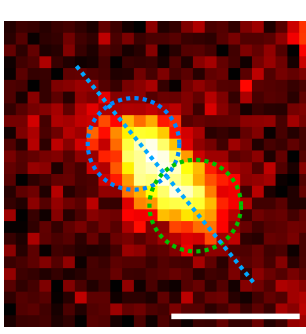

(a) Summe

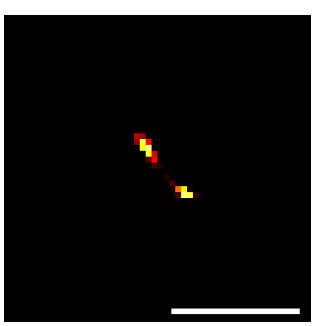

(c) SPEED

Rekonstruktion

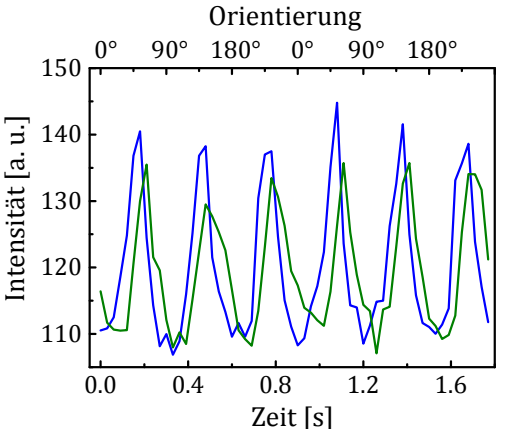

(b) Fluoreszenzintensität

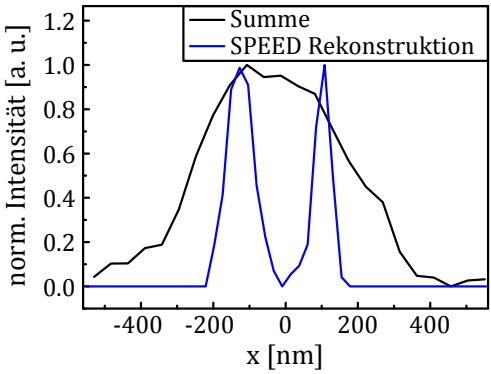

(d) Intensitätsprofil

Abbildung 1.22: Messung von Atto 590 Molekülen mit ExPAN. a: Summe von 60 Einzelbildern der Messung. b: Mittlere Fluoreszenzintensität für die beiden in a markierten Flächen. c: Ergebnis der SPEED-Rekonstruktion mit ExPAN $\left(\lambda_{1}=1, \lambda_{2}=1,500\right.$ Iterationen). d: Intensitätsprofil entlang der in a eingezeichneten Gerade. (Maßstab: $500 \mathrm{~nm}$ )

tung auf wenige Bildpunkte zurückgeführt und die meisten Moleküle eindeutig voneinander separiert werden.

Die Punktdichte der Rekonstruktion auf der linken Seite ist identisch mit der der Rohdaten (50 nm pro Bildpunkt), sodass einige Moleküle nur durch ein oder zwei helle Bildpunkte repräsentiert werden. Der SPEED-Algorithmus ist in der Lage eine Rekonstruktion mit einem ganzzahligen Vielfachen der Auflösung der Ausgangsdaten zu erzeugen. Auf der rechten Seite in Abbildung 1.21 ist das Ergebnis der SPEED-Rekonstruktion mit identischen Parametern, jedoch der doppelten Auflösung dargestellt. An der markierten Stelle ist hier eindeutig die Separation in zwei Signale zu erkennen. Der Abstand der beiden Punkte ist dabei mit $\sim 80 \mathrm{~nm}$ deutlich unterhalb der theoretischen Aufösungsgrenze von $\sim 230 \mathrm{~nm}$.

\subsubsection{Auswertung mit ExPAN}

Die Abbildung 1.22 zeigt eine Messung von zwei Atto 590 Molekülen mit ExPAN. Rechts oben ist der Signalverlauf für die in a markierten Flächen aufgetragen. Die Signale weisen 
eine Phasenverschiebung von lediglich $\sim 20^{\circ}$ auf. Für die SPEED-Rekonstruktion, die in $\mathbf{b}$ dargestellt ist, wurde die schmalere Winkelabhängigkeit des Fluoreszenzsignals durch die Modulationsfunktion mit ExPAN nach Gleichung 1.37 berücksichtigt. Obwohl die Signale eine deutliche zeitliche und räumliche Überlagerung aufweisen, konnten die Moleküle durch die SPEED-Rekonstruktion vollständig voneinander separiert werden. Wie erwartet scheint die größere Photoselektion durch ExPAN somit eine verbesserte Rekonstruktion bei Signalen mit ähnlicher Phase zu ermöglichen.

\subsubsection{Simulationen von Einzelmoleküldaten}

Die Fähigkeit des Algorithmus das Signal zweier Moleküle voneinander zu trennen hängt von deren Abstand und relativer Orientierung ab. Um die Qualität der Rekonstruktion bei unterschiedlichen Bedingungen zu evaluieren, wurden verschiedene Simulationen durchgeführt. Signalintensität und Hintergrundrauschen wurden dabei entsprechend der gemessenen Daten aus Abbildung 1.21 modelliert. Für die Abbildung 1.23 wurden Paare von Emittern mit verschiedenen Abständen und unterschiedlichen relativen Orientierungen simuliert und unter den selben Bedingungen ausgewertet, die auch für die Messungen verwendet wurden. Als Punktspreizfunktion wurde eine Gaußverteilung mit einer Halbwertsbreite von 5 Bildpunkten benutzt, was bei $50 \mathrm{~nm}$ pro Bildpunkt einer Halbwertsbreite von $250 \mathrm{~nm}$ entspricht. Der Graph in Abbildung 1.23 zeigt das horizontale Intensitätsprofil der simulierten Daten (schwarz) und der Auswertung (blau). Erwartungsgemäß lassen sich die Signale von Emittern mit einem Abstand größer als der Halbwertsbreite der PSF (300 nm, 400 nm) in jedem Fall vollständig trennen. Bei einem Abstand von 200 nm können die Emitter auch bei ähnlicher Orientierung noch aufgelöst werden. Bei $100 \mathrm{~nm}$ wird die Separation mit abnehmender Winkeldifferenz schlechter und ab einer Differenz von $30^{\circ}$ ist eine Auflösung der Emitter nicht mehr möglich, allerdings zeigt sich eine Schärfung vergleichbar einer herkömmlichen Dekonvolution.

Die Simulation eines komplexeren Szenarios ist in Abbildung 1.24 illustriert. Hier wurde eine Gruppe von 64 Emittern bei drei verschiedenen Dichten simuliert. Die Positionen und Orientierungen der Emitter wurden zufällig über eine gleichverteilte Zufallsvariable gewählt. Neben dem Summen- und Phasenbild ist die reale Emitterverteilung dargestellt. Um die Qualität der Rekonstruktion zu quantifizieren, bietet es sich an die Abweichung zwischen Rekonstruktion und exakter Emitterverteilung zu bestimmen. Zu diesem Zweck wurde die Wurzel der mittleren quadratischen Abweichung (root-mean-square error, RMSE) zwischen der Rekonstruktion und der exakten Verteilung berechnet. Je kleiner der resultierende Wert, desto besser gibt die Rekonstruktion das exakte Bild wieder. 


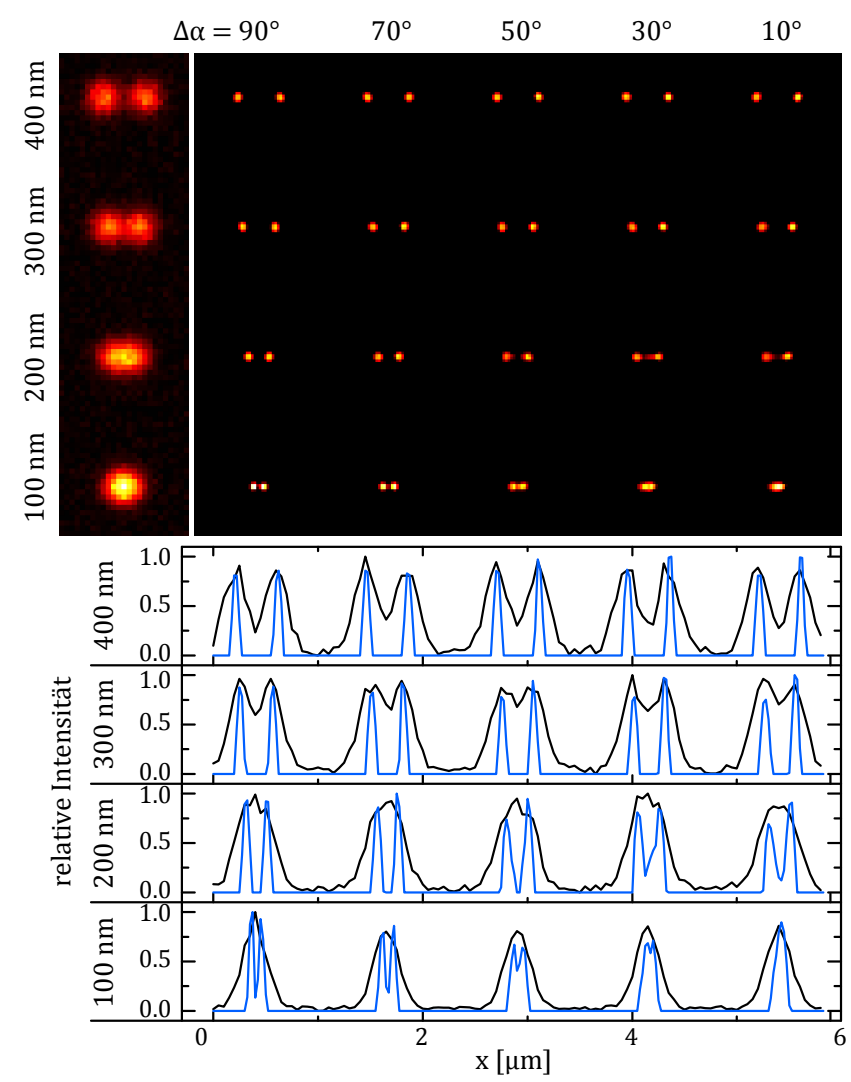

Abbildung 1.23: SPEED-Rekonstruktion von simulierten Molekülpaaren mit unterschiedlichen Abständen sowie unterschiedlichen relativen Orientierungen. Oben: Ergebnis der Rekonstruktion ( $\lambda_{1}=1, \lambda_{2}=1,500$ Iterationen) und Ausschnitt der Simulation. Unten: Intensitätsprofil in horizontaler Richtung für die Simulation (schwarz) und die SPEED-Rekonstruktion (blau).

Bei der geringsten Dichte $\left(7.1 \mathrm{\mu m}^{-2}\right)$ in Abbildung 1.24 konnte der SPEED-Algorithmus den größten Teil der Emitter auflösen und es sind nur vereinzelt Emitter-Paare zu erkennen, die nicht separiert wurden. Mit steigender Dichte nimmt die Qualität der Rekonstruktion sichtbar ab, was sich ebenfalls in der Zunahme der mittleren quadratischen Abweichung manifestiert. Besonders bei der höchsten Dichte $\left(28 \mu^{-2}\right)$ zeigt sich dabei ein Zusammenlaufen benachbarter Signale.

In Abbildung 1.24 ist darüber hinaus die SPEED-Rekonstruktion einer Simulation ohne Modulation dargestellt. Es hat sich gezeigt, dass der SPEED-Algorithmus prinzipiell auch ohne Modulation Strukturen unterhalb der Beugungsgrenze auflösen kann. Bei geringer Emitterdichte können somit auch ohne Modulation viele Emitter aufgelöst werden. Verglichen mit der modulierten Simulation weist die Rekonstruktion allerdings eine deutlich geringerer Qualität auf und besitzt eine dementsprechend große quadratische Abweichung. Dabei sind besonders bei hoher Dichte signifikante Unterschiede zu der realen Anordnung der Emitter 


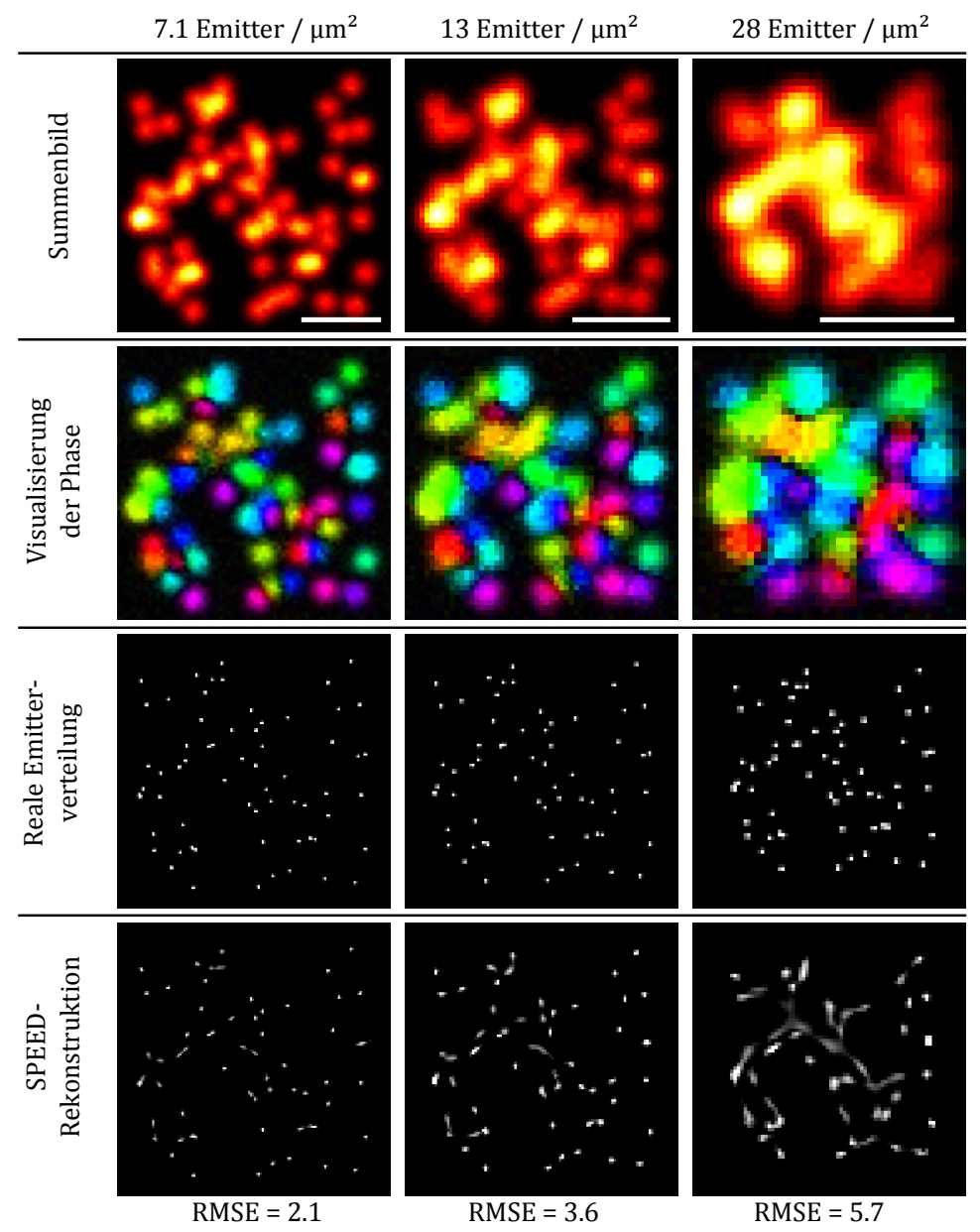

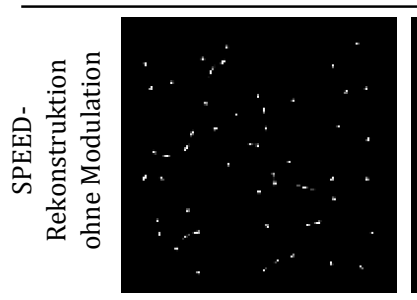

RMSE $=4.7$

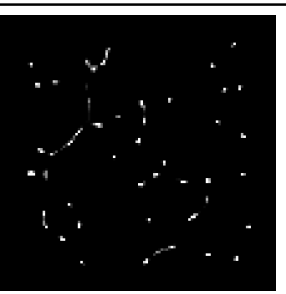

RMSE $=6.4$

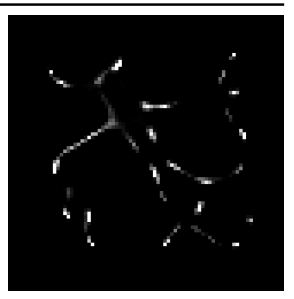

RMSE $=9.2$

Abbildung 1.24: Simulation von 64 Molekülen mit zufälliger Anordnung und Orientierung bei 3 verschiedenen Dichten. Die Abbildung zeigt (von oben nach unten) die Summe der simulierten Einzelbilder, die Visualisierung der Phase, die reale Verteilung der Emitter durch eine lineare Interpolation der Emitterpositionen auf ein Pixelraster mit doppelter Auflösung, die SPEEDRekonstruktion mit zweifachem Oversampling und die SPEED-Rekonstruktion einer analogen Simulation ohne Modulation. (Maßstab: $1 \mu \mathrm{m}$ )

zu erkennen. Ursache für diese Verhalten ist, dass der SPEED-Algorithmus in jedem Fall eine dünn besetzte Lösung anstrebt. Ohne Modulation gibt es jedoch keine dünne Beset- 
zung in der Phasen- bzw. Zeitdomäne, die fehlende Information führt dadurch vor allem bei höheren Emitterdichten zu einer weniger akkuraten Ergebnis.

\subsubsection{Simulationen von Einzelmoleküldaten mit ExPAN}

Um den Einfluss des ExPAN-Effekts auf die SPEED-Rekonstruktion genauer zu untersuchen, wurden Simulationen durchgeführt, bei denen das Signal entsprechend Gleichung 1.37 mit ExPAN modelliert wird. Die Intensität der stimulierten Emission wird dabei durch den Parameter $f_{s}$ bestimmt, wobei $f_{s}=0$ der normalen $\cos ^{2}$-Modulation entspricht. In Abbildung 1.25 sind die Ergebnisse der Rekonstruktion von Simulationen, analog zu der in Abbildung 1.24, bei zwei unterschiedlichen Parametern für die ExPAN-Intensität $f_{s}$ illustriert. Zum Vergleich sind die reale Emitterverteilung und das Ergebnis der Rekonstruktion ohne ExPAN dargestellt. Die Simulationen weisen eine verbesserte Rekonstruktion mit zunehmender ExPAN-Intensität auf, wobei Unterschiede am deutlichsten bei hoher Emitterdichte zu erkennen sind. Zudem nimmt die mittlere quadratische Abweichung zur realen Emitterverteilung ebenfalls mit zunehmender ExPAN-Intensität ab. Das bestätigt, dass die SPEED-Rekonstruktion bei hoher Emitterdichte und ähnlicher Orientierung durch den Einsatz von ExPAN verbessert werden kann.

In Abbildung 1.26 ist mittlere quadratische Abweichung von Simulationen mit unterschiedlicher ExPAN-Intensität gegen $f_{s}$ aufgetragen. Alle übrigen Parameter für die Simulation und für die Auswertung wurden konstant gehalten. Die Auftragung zeigt, dass die Rekonstruktion bei kleinem $f_{s}$ mit zunehmender ExPAN-Intensität schnell besser wird. Ab einer ExPAN-Intensität von 20 stagniert die Abweichung jedoch und scheint bei sehr hoher Intensität sogar wieder leicht anzusteigen. Dieses Verhalten ist durch die Verringerung der Gesamtintensität mit zunehmenden Wert für $f_{s}$ zu erklären, da durch die schmalere Winkelverteilung über den gesamten Winkelbereich betrachtet weniger Fluoreszenz emittiert wird. Ab einem gewissen Punkt hebt das schlechtere Signal-Rausch-Verhältnis die Verbesserung durch den ExPAN-Effekt auf. Es gibt folglich eine optimale ExPAN-Intensität für die SPEED-Rekonstruktion, die von der Helligkeit des Farbstoffs und der Größe des Rauschanteils abhängt. 


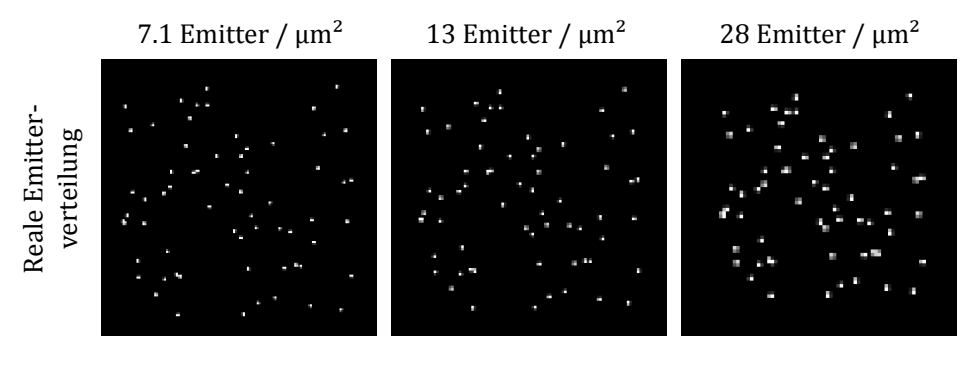

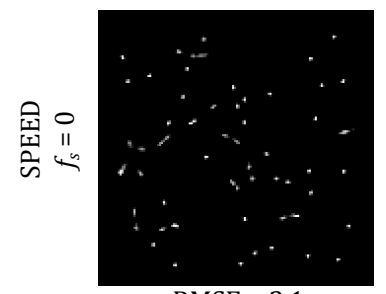

RMSE $=2.1$

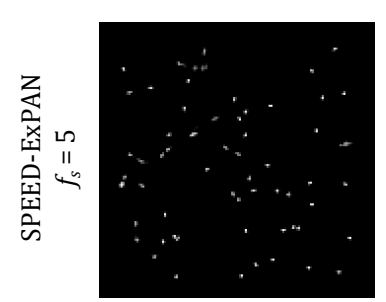

RMSE $=1.9$

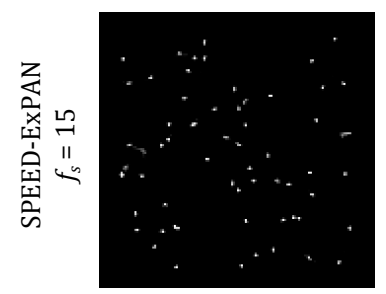

RMSE $=1.7$

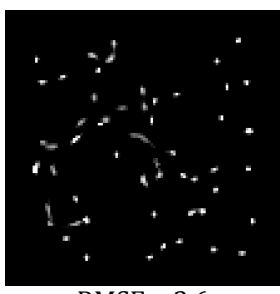

RMSE = 3.6

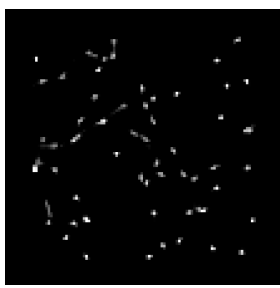

RMSE $=2.9$

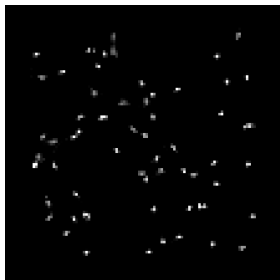

RMSE $=2.7$

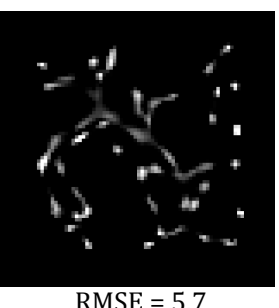

RMSE $=5.7$

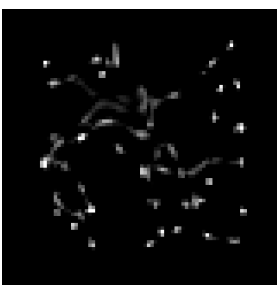

RMSE $=5.0$

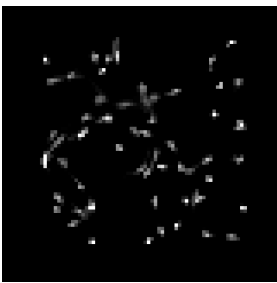

RMSE $=4.5$

Abbildung 1.25: Simulationen analog zu denen in Abbildung 1.24 bei unterschiedlicher ExPANIntensität $f_{s}$. In der ersten Zeile ist die reale Verteilung der Emitter illustriert, darunter die SPEED-Rekonstruktion von Simulationen mit $f_{s}=0, f_{s}=5$ und $f_{s}=15$.

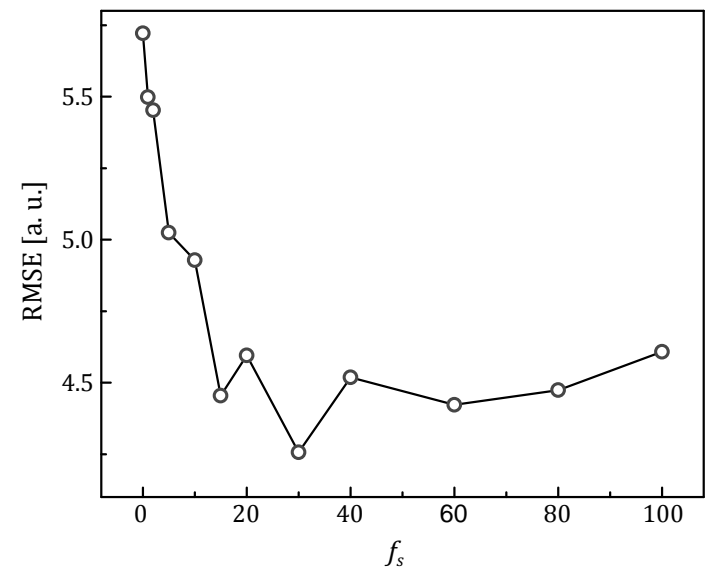

Abbildung 1.26: Mittlere quadratische Abweichung zwischen der exakten Anordnung der Emitter und der rekonstruierten Intensitätsverteilung von Simulationen mit unterschiedlichen Werten für den Parameter $f_{s}$. Die Simulationen entsprechen denen aus Abbildung 1.25 bei einer Emitterdichte von $28 \mathrm{\mu m}^{-2}$. 


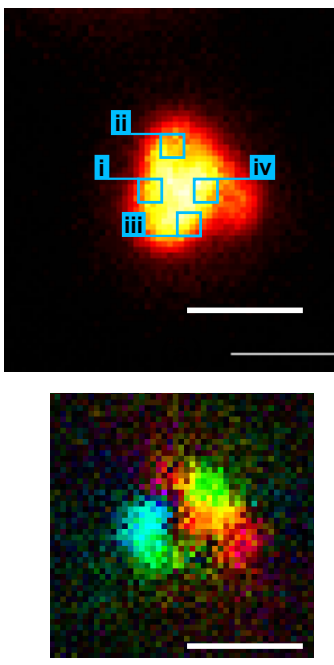

(b)

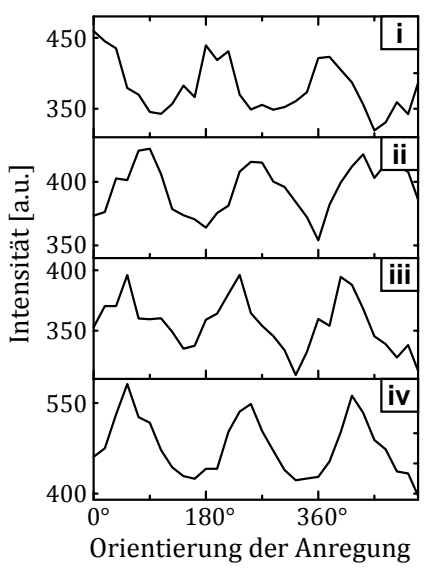

(c)

Abbildung 1.27: Messung von Atto 590 gelabelten Microspheres mit einem Durchmesser von 500 nm. a: Summe von 10 Einzelbildern der Messung. b: Visualisierung der Fourier-Koeffizienten der Modulationsfrequenz c: Intensitätsverlauf für die in a markierten Flächen. (Maßstab: 1 um)

\subsubsection{Proben mit höherer Farbstoffdichte}

Im folgenden werden zwei Beispiele für Proben mit höherer Farbstoffdichte vorgestellt, die eine Modulation der Fluoreszenzintensität aufweisen und bei denen die Gewinnung lokalspezifischer Phaseninformation möglich ist.

\subsubsection{Microspheres}

Polystyrol-Microspheres haben eine gut definierte Form sowie Größe und werden häufig als Kalibrationsstandard verwendet, zum Beispiel zur Bestimmung der PSF eines Fluoreszenzmikroskops [63]. In dieser Arbeit wurden die Microspheres als vereinfachtes Modellsystem für strukturierte Proben, wie beispielsweise Membran-markierte Zellen eingesetzt. Dazu wurden mit Streptavidin funktionalisierte Polystyrol-Micospheres verwendet, die vor der Messung mit Biotin-konjugiertem Atto 590 Farbstoff markiert wurden. Auf diesem Weg war es möglich die Micospheres nur an deren Oberfläche zu markieren und eine moderate Farbstoffdichte zu erreichen.

In Abbildung 1.27 ist der Ausschnitt einer Messung gezeigt, auf der drei aneinandergelagerte Microspheres zu erkennen sind. Die Dichte der Farbstoffmoleküle ist hier größer, als bei den zuvor gezeigten Messungen. Trotzdem weisen die Microspheres eine Modulation des Fluoreszenzsignals mit räumlich variierender Phase auf, wie an den Intensitätsverläufen in 
Abbildung 1.27c sowie den Fourier-Koeffizienten in Abbildung 1.27b zu erkennen ist. Durch die Überlagerung der Signale von vielen Emittern ist die relative Amplitude hier jedoch kleiner als bei den Messungen von Einzelmolekülen.

Unter der Annahme, dass die Farbstoffmoleküle auf der Oberfläche eine zufällige Orientierung besitzen und in etwa die gleiche Fluoreszenzintensität aufweisen, so ist die Gesamtamplitude von $N$ Emittern im Mittel proportional zu $\sqrt{N}^{(9)}$. Die mittlere Gesamtintensität des Signals ist hingegen proportional zu $N$. Aus diesem Grund nimmt die relative Amplitude bei zunehmender Emitterdichte mit $1 / \sqrt{N}$ ab. Die Phase des Signals resultiert dabei aus der mittleren Orientierung der Emitter.

Darüber hinaus müssen Diffusionseffekte beachtet werden. Der Farbstoff ist über einen relativ kurzen, jedoch flexiblen $\mathrm{C}_{5}$-Linker an das Biotin-Molekül gebunden, wodurch die Farbstoffmoleküle in einem beschränkten Winkelbereich Pendel- und Rotationsbewegungen ausführen können. Rotationskorrelationszeiten von vergleichsweise kleinen Molekülen wie Atto 590 liegen im Bereich von mehreren 100 ps [65]. Das gemessene Signal entsteht folglich aus einer Mittlung der möglichen Orientierungen und weist somit eine geringer Modulationsamplitude auf, als ein fixes Molekül. Wie Abbildung 1.27b zeigt, kann bei ausreichendem Signal-Rausch-Verhältnis auch bei geringer Amplitude Phaseninformation aus den Daten gewonnen werden. Grundsätzlich sollte es daher möglich sein, die Phase zu nutzen, um auch bei dichteren Proben Strukturen unterhalb der Beugungsgrenze zu unterschieden.

\subsubsection{Mikrotubuli in fixierten Hippocampus-Neuronen}

Als Beispiel für biologische Proben wurden Messungen mit fixierten Hippocampus-Neuronen mit fluoreszenzmarkierten Mikrotubuli durchgeführt. Mikrotubuli sind Teil des Cytoskeletts eukaryotischer Zellen und sind aus zwei Proteinmolekülen ( $\alpha$ - und $\beta$-Tubulin) aufgebaut, die zu filamentösen Strukturen polymerisieren [66]. Abbildung 1.28 zeigt die Messung einer Probe von fixierten Hippocampus-Neuronen aus dem Gehirn einer Ratte, bei der $\beta 3$ Tubulin mit dem Farbstoff Cy3 immunomarkiert wurde [42]. Die Visualisierung der FourierKoeffizienten in Abbildung 1.28b zeigt eindeutig, dass auch bei diesen Proben eine Modulation der Fluoreszenzintensität zu beobachten ist. Die Phase variiert dabei scheinbar zufällig innerhalb der Probe. Bedingt durch die höhere Farbstoffdichte ist die Amplitude, verglichen mit den Einzelmolekül-Messungen auch hier deutlich geringer. Für die beiden aufgetragenen Signale beträgt die Amplitude $\sim 10 \%$ der mittleren Intensität. Um ein ausreichendes

\footnotetext{
${ }^{(9)}$ Der Erwartungswert der Amplitude für ein Signal aus $N$ Sinusoiden mit gleicher Frequenz und zufälliger Phase lässt sich auf ein zweidimensionales Random-Walk-Problem zurückführen. Bei gleicher Amplitude entspricht der Erwartungswert dem einer Rayleigh-Verteilung mit $\sigma=\sqrt{N / 2}$ und ist somit proportional zu $\sqrt{N}$ [64].
} 


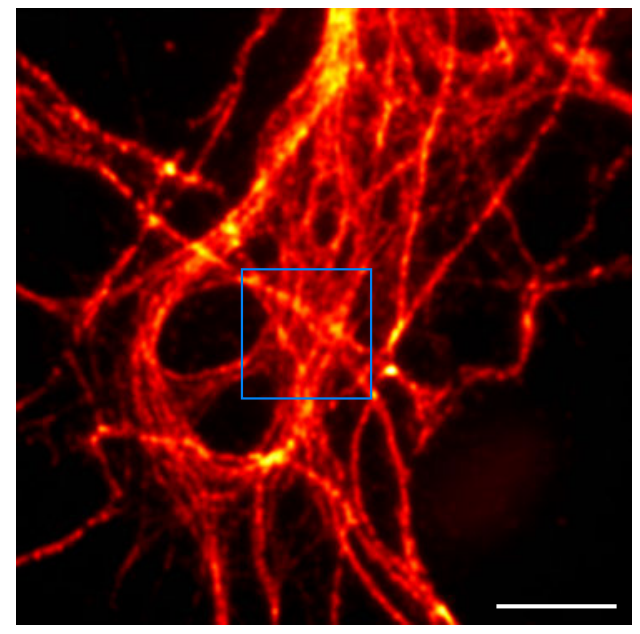

(a) Weitfeldaufnahme

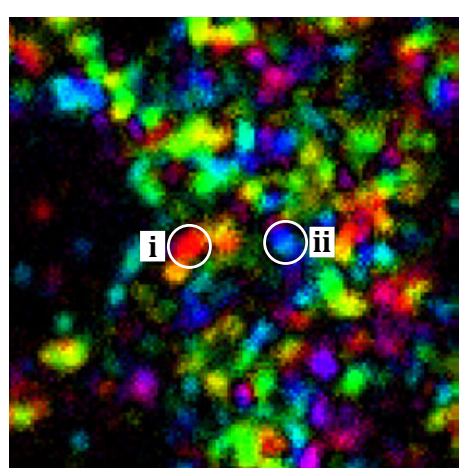

(c) Fourier-Visualisierung Vergrößerung

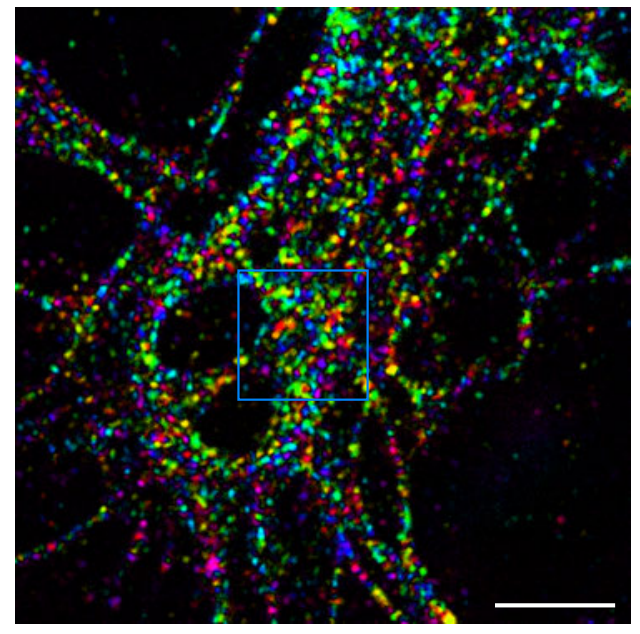

(b) Fourier-Visualisierung

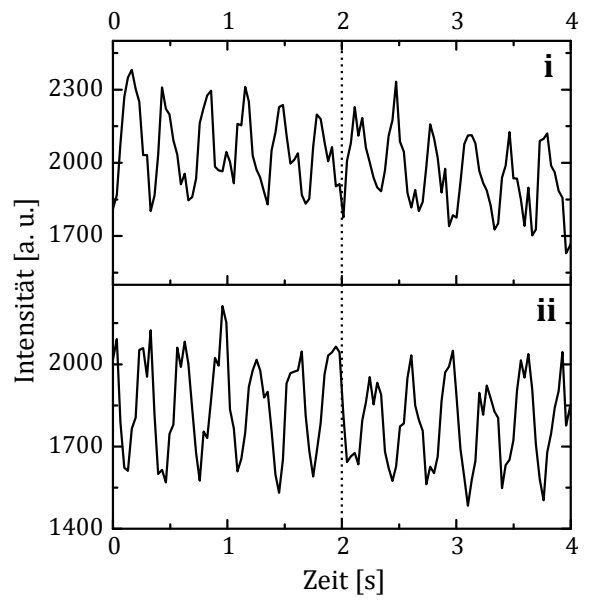

(d) Intensitätsverlauf

Abbildung 1.28: Hippocampus-Neuron einer Ratte mit Cy3 immunomarkiertem $\beta 3$ Mikrotubulin. a: Summe von 80 Einzelbildern der Messung. b: Visualisierung der FourierKoeffizienten. c: Vergrößerung $(5 \mu \mathrm{m} \times 5 \mu \mathrm{m})$ des in $\mathbf{b}$ markierten Bereichs. d: Mittlere Fluoreszenzintensität für die beiden in c markierten Bereiche.(Maßstab: $5 \mu \mathrm{m}$ )

Signal-zu-Rausch-Verhältnis zu erreichen, wurden für die Abbildung 1.28b 800 Einzelbildern der Messung ausgewertet. Die Fixierung der Probe mit Polyvinylalkohol unterbindet die Rotationsdiffusion nicht vollständig [67]. Aus diesem Grund ist anzunehmen, dass die Amplitude zusätzlich durch eine eingeschränkte Rotationsdiffusion der Farbstoffmoleküle verringert wird. 


\subsection{Fazit}

In diesem Teil der Arbeit konnte erstmals gezeigt werden, dass es möglich ist die Winkelselektivität der Fluoreszenzanregung durch stimulierte Emission zu vergrößern. Die Verbesserung der Photoselektion durch ExPAN wurde experimentell an Einzelmolekülen demonstriert und zeigte eine gute Übereinstimmung mit der theoretischen Beschreibung. Die Methode könnte neben der hier untersuchten Anwendung, vor allem im Kontext von FluoreszenzanisotropieExperimenten oder bei der Bestimmung der molekularen Orientierung, z.B. von fluoreszenzmarkierten Proteindomänen, von Interesse sein. Vor allem die Fluoreszenzanisotropie findet in den Biowissenschaften breite Anwendung und wird z.B. beim Studium von RezeptorLiganden-Interaktionen [68] oder der Proteindynamik [69] genutzt. Hierbei könnte ExPAN zur Vergrößerung der Anisotropie eingesetzt werden. Vorstellbar wäre z.B. ein zeitaufgelöstes Fluoreszenzdepolarisations-Experiment mit gepulster Anregung, bei dem ein zweiter ExPAN-Laserpuls mit orthogonaler Polarisation stimulierte Emission induziert. Auf diesem Weg sollte es möglich sein eine deutlich höhere Anfangspolarisation zu erzeugen und so den Dynamikumfang der Messung zu vergrößern.

In jüngerer Vergangenheit wurden verschiedene Methoden vorgestellt, die Polarisationsmikroskopie einsetzen, um die Winkelverteilung von Fluorophoren auf Membranen zu untersuchen [70, 71]. Auch für diese Methoden könnte eine Vergrößerung der Photoselektion durch ExPAN von Vorteil sein.

Im Rahmen der Arbeit wurde die Anwendung von ExPAN in Verbindung mit einem neuen Mikroskopie-Ansatz untersucht, bei dem die Orientierung von Farbstoffmolekülen als Kontrastmechanismus genutzt wird. Die Rotation der Anregungspolarisation im Weitfeld erwies sich dabei als robuste Methode zur Bestimmung der azimutalen Orientierung von oberflächengebundenen Farbstoffmolekülen. Es konnte gezeigt werden, dass die zusätzliche Information zur Unterscheidung von Molekülen genutzt werden kann, selbst wenn sich deren Signale räumlich überlagern. Durch den SPEED-Algorithmus konnten dabei Moleküle mit einem Abstand von bis zu 80 nm erfolgreich separiert und lokalisiert werden, wobei die Qualität der Rekonstruktion von der relativen Orientierung der Emitter abhängt und bei ähnlicher Ausrichtung sowie zunehmender Emitterdichte abnimmt. Anhand von Simulationen konnte gezeigt werden, dass der Einsatz von ExPAN die Qualität der SPEED-Rekonstruktion bei höheren Emitterdichten signifikant vergrößert. Ein moduliertes Fluoreszenzsignal konnte auch bei oberflächenmarkierten Mikropartikeln in wässriger Lösung sowie bei fixierten biologischen Proben beobachtet werden. 



\section{Konfokale}

\section{Multidetektor-Scanning-Mikroskopie}

\section{$2.1 \quad$ Einleitung}

Dieses Kapitel der Arbeit widmet sich einem neuartigen Ansatz für die Realisierung eines sog. Image-Scanning-Mikroskops. Der Ausdruck Image-Scanning-Mikroskopie (ISM) wurde von Jörg Enderlein geprägt, der im Jahr 2010 erstmals ein entsprechendes Mikroskop vorgestellte [72]. Das Konzept wurde bereits im Jahr 1988 von Colin J. R. Sheppard theoretisch beschrieben [73] und ist verwand mit der Structured-Illumination-Mikroskopie [74]. Die Methode basiert auf der Abhängigkeit der Auflösung eines Konfokalmikroskops von der Größe der verwendeten Lochblende bzw. Detektorapertur. Bei der Image-Scanning-Mikroskopie wird der Detektor eines Konfokalmikroskops durch eine Kamera substituiert, und anstelle eines einzelnen Messwerts ein Bild der Intensitätsverteilung für jeden Punkt der Probe aufgenommen. Dabei wirkt jedes Pixel der Kamera als Detektor mit sehr kleiner Apertur. Sheppard und Enderlein konnten zeigen, dass auf diesem Weg ein Bild mit signifikant verbesserter Auflösung gewonnen werden kann [72, 75]. Verglichen mit einer entsprechenden Weitfeld-Aufnahme kann ISM theoretisch eine bis zu doppelt so hohe laterale Auflösung erreichen.

Alle bisher vorgestellten Image-Scanning-Mikroskope verwenden CCD- oder CMOS-Kameras als Flächendetektoren. Besonders die begrenzte Aufnahmegeschwindigkeit der Kameras war dabei zunächst ein limitierender Faktor. Da für jeden Punkt ein separates Bild aufgenommen werden muss, kann die Erfassung des gesamten Bildbereichs viele Sekunden beanspruchen. Schulz et al. konnten zeigen, dass eine Parallelisierung der Datenerfassung mit einem Confocal-Spinning-Disk-Mikroskop (CSD) möglich ist [76]. Auf diesem Weg können viele Anregungsfoki gleichzeitig in einem Bild aufgenommen werden. Damit die Intensitätsverteilung dabei nicht auf der Kamera verschmiert wird, muss die Anregung gepulst und synchronisiert mit der Rotation der Scheibe des CSD erfolgen. Mit dieser Methode sind Aufnahmegeschwin- 
digkeiten in der Größenordnung von wenigen Sekunden möglich. Ein ähnlicher Ansatz wurde von York et al. verfolgt, bei dem ein Gitter von Anregungspunkten durch Mikrospiegelaktoren erzeugt und über den Bildbereich bewegt wird [77]. Bei diesen Methoden besteht der aufgenommene Datensatz aus einer Reihe von Einzelbildern, die in einem Nachbearbeitungsschritt zu einem ISM-Bild kombiniert werden müssen.

Noch höhere Geschwindigkeiten lassen sich erreichen, wenn die Intensitätsverteilung für jeden Anregungspunkt schon während der Aufnahme an die richtige Position auf der Kamera abgebildet wird. Dazu wird das fokussierte Anregungslicht, analog zu einem gewöhnlichen konfokalen Laser-Scanning-Mikroskop (Confocal Laser Scanning Microscope, CLSM), mit Scannerspiegeln durch die Probe bewegt. Das Fluoreszenzlicht wird, nachdem es eine Lochblende passiert hat, durch ein zweites Scannersystem an der korrekten Position auf der Kamera abgebildet [78]. Diese Technik wurde ebenfalls in einer parallelen Variante mit vielen Anregungsfoki unter dem Namen Multifocal Structured Illumination Microscopy (MSIM) von York et al. vorgestellt [79]. Hierdurch sind Aufnahmen mit Video-Geschwindigkeit möglich, allerdings ist der erforderliche optische Aufbau sehr komplex und aufwendig zu justieren.

Die hier vorgestellte Methode verwendet anstelle einer CCD-Kamera eine Reihe von Lawinenphotodioden (single-photon avalanche diode, SPAD) als Detektoren. Lawinenphotodioden, die teilweise auch in kommerziellen CLSM-Systemen eingesetzt werden, besitzen eine hohe Zeitaufösung ( $<1 \mathrm{~ns})$ und benötigen im Gegensatz zu einer Kamera keine Integrationszeit. Jedes detektierte Photon wird direkt in ein elektrisches Signal umgesetzt, was eine schnelle und kontinuierliche Abtastung zulässt. Es existieren kommerzielle und experimentelle Detektoren [80, 81], bei denen mehrere Lawinenphotodioden in einem Bauteil zusammengefasst sind. Allerdings ist der Anteil der lichtempfindlichen Fläche bei diesen Detektoren aus technischen Gründen sehr gering, sodass bei einem Einsatz als Flächendetektor ein Großteil des Signals verloren gehen würde. Aus diesem Grund wird in der vorliegenden Arbeit ein Glasfaserbündel verwendet, um eine Reihe von separaten SPAD-Modulen zu einem Detektor zu kombinieren. Das Faserbündel wird anstelle der Lochblende in den Strahlengang eines gewöhnlichen CLSM integriert. Die Anwendung des ISM-Prinzips auf diese Art der Detektion wird im Folgenden als Multi-Detektor-Scanning-Mikroskopie (MDS) bezeichnet.

In Gegensatz zu kamerabasierten ISM-Varianten kann MDS problemlos in Kombination mit Fluoreszenzlebensdauer-Mikroskopie (fluorescence lifetime imaging microscopy, FLIM) eingesetzt werden. Eine Datenerfassung durch zeitkorrelierte Einzelphotonenzählung ermöglicht dabei eine simultane Messung von Fluoreszenz-Abklingkurven. FLIM ist eine vielseitige Methode zur Sondierung der molekularen Umgebung von Fluorophoren und hat in den letzten beiden Jahrzehnten in den Biowissenschaften zunehmend an Bedeutung gewonnen. Abhän- 
gig vom eingesetzten Fluorophor, ermöglicht FLIM Einblicke in die räumliche Verteilung von Parametern wie Calcium-Konzentration [82, 83], pH-Wert [84, 85] oder Membranstruktur und Komposition [86, 87]. Zudem kann die Fluoreszenzlebensdauer von metabolischen Coenzymen wie $\mathrm{NADH}^{(10)}$ kann Aufschluss über deren Redoxzustand geben und wird genutzt, um den Zellmetabolismus zu Untersuchen. Auf diesem Weg ist es z.B. möglich kanzerose Zellen zu identifizieren [88]. Von besonderer Bedeutung ist FLIM für Protein-Protein-Interaktionsstudien, die auf dem Förster-Resonanzenergietransfer (FRET) beruhen [89]. Im Gegensatz Fluoreszenzintensität ist die Lebensdauer größtenteils unabhängig von der - zumeist unbekannten - Konzentration der Fluorophore, was für viele Anwendungen ein entscheidender Vorteil ist. Trotz der beträchtlichen Bedeutung der Methode wird FLIM bisher kaum mit hochaufgelösten Mikroskopieverfahren eingesetzt. Die Verbesserung der räumlichen Auflösung von FLIM mit MDS könnte somit für eine ganze Reihe von biologischen Fragestellungen von Interesse sein.

(10) Nicotinamidadenindinukleotid 


\subsection{Theoretische Grundlagen}

\subsubsection{Konfokale Laser-Scanning-Mikroskopie}

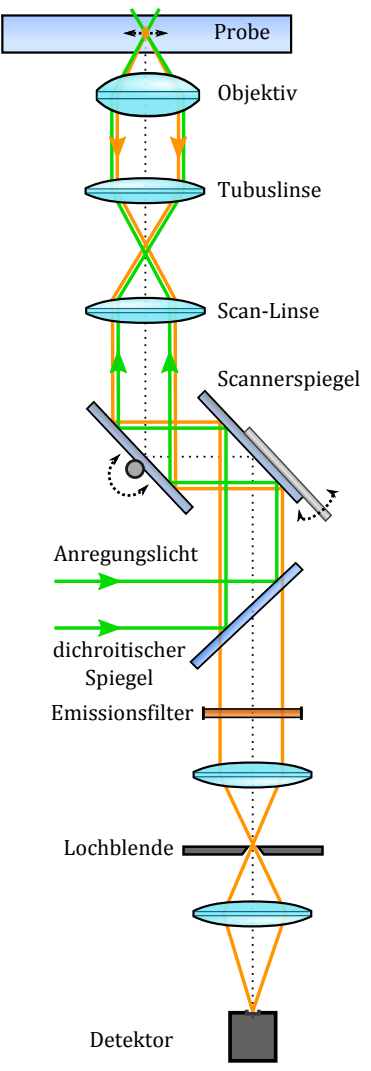

Abbildung 2.29: Aufbau eines konfokalen LaserScanning-Mikroskops.
Ein Konfokalmikroskop unterscheidet sich von einem Weitfeldmikroskop in erster Linie dadurch, dass nicht die gesamte Probe beleuchtet wird, sondern zu jedem Zeitpunkt nur ein kleiner Bereich. Um ein vollständiges Bild zu erhalten, wird der Fokuspunkt rasterförmig durch die Probe bewegt und das Bild dabei Punkt für Punkt aufgebaut. Der optische Aufbau eines Konfokalmikroskop erlaubt die Installation einer Lochblende in der Bildebene, die verhindert, dass Licht von außerhalb der Fokusebene auf den Detektor trifft. Diese räumliche Filterung erhöht zum einem die Auflösung in axialer Richtung und verringert zum anderen das Hintergrundsignal. Das dadurch erreichte sog. optical sectioning ermöglicht es optische Schnittbilder mit hohem Kontrast aufzunehmen. Bereits in den 50er Jahren hat Marvin Minsky ein Mikroskop nach diesem Prinzip vorgestellt [90]. In der Weiterentwicklung zum konfokalen Laser-Scanning-Mikroskop (confocal laser scanning microscope, CLSM) finden dieses heute breite Anwendung in den Biound Materialwissenschaften.

Die Abtastung der Probe kann technisch auf unterschiedliche Weise erfolgen. Im einfachsten Fall wird die Probe durch eine verfahrbaren Probentisch bewegt, wobei der Strahlengang im Mikroskop unverändert bleibt (stage-scanning). Das hat den Vorteil, dass viele mögliche Abbildungsfehler (z.B. Astigmatismus) des Objektives keinen Einfluss auf das aufgenommene Bild haben und der optische Aufbau relativ simpel ist. Allerdings ist die Geschwindigkeit mit der der Probentisch bewegt werden kann durch dessen Masse begrenzt, sodass die Datenerfassung vergleichsweise lange dauert.

Eine andere Möglichkeit besteht in der Verwendung von beweglichen Scannerspiegeln im Strahlengang des Mikroskops (beam-scanning), die den Punkt der Anregung durch die Probe bewegen. Dabei wird in der Regel ein schneller Scanner-Spiegel für die Abtastung der Zeilen und ein, sich senkrecht dazu bewegender, langsamer Spiegel für den Zeilenvorschub 
verwendet. Abhängig von der Geschwindigkeit der eingesetzten Scanner lassen sich auf diesem Weg Bildraten mit Videogeschwindigkeit und schneller erreichen.

In Abbildung 2.29 ist der Strahlengang eines typischen beam-scanning CLSM schematisch illustriert. Das Licht für die Fluoreszenzanregung wird hierbei nach der Einkoppelung über einen dichroitischen Spiegel von zwei beweglichen Scanner-Spiegeln abgelenkt. Durch ein Linsensystem aus Scan- und Tubuslinse werden die Scanner-Spiegel auf die Rückapertur des Objektives abgebildet. Bei einer Rotation der Spiegel ändert sich dadurch der Einfallswinkel des Anregungslichts in der telezentrischen Ebene des Objektives, was wiederum zu einer lateralen Verschiebung des Anregungspunkts in der Fokusebene führt. Das Fluoreszenzlicht aus dem Anregungspunkt durchläuft den selben Weg in entgegengesetzter Richtung, wodurch sich die Ablenkung durch die Scanner-Spiegel wieder aufhebt. Durch optische Filter wird das Fluoreszenzlicht von dem Anregungslicht getrennt und durch eine Linse fokussiert. In der dabei entstehenden Bildebene ist eine Lochblende platziert. Licht von außerhalb der Fokusebene ist hier nicht fokussiert und kann die Blende nicht passieren. Dahinter befinden sich ein oder auch mehrere Detektoren, abhängig von der Anzahl der spektralen Kanäle. Der Detektor muss neben einem hohen Signal-Rausch-Verhältnis vor allem eine schnelle Reaktionszeiten aufweisen, deshalb werden hier zumeist Photomultiplier-Tubes oder Photodioden eingesetzt. Das analoge Ausgabesignal des Detektors wird schließlich mithilfe eines sog. Framegrabbers digitalisiert und zu einem Bild zusammengesetzt [91].

\subsubsection{Abbildungseigenschaften eines CLSM}

Wie in Kapitel 1.2.4 erläutert, beschreibt die Punktspreizfunktion (PSF) eines optischen Systems die Abbildung eines idealen punktförmigen Objektes. Für ein Weitfeld-Mikroskop kann die PSF für ein Objekt im Fokus als Beugungsscheibe beschrieben werden (sieh nach Gleichung 1.11). Bei einem Konfokalmikroskop wird das Anregungslicht auf einen Punkt fokussiert und besitzt, im Unterschied zu einem Weitfeld-Mikroskops, eine inhomogene Intensitätsverteilung. Aus diesem Grund setzt sich die effektive PSF eines konfokalen FluoreszenzMikroskops $U_{\text {con }}$ aus der PSF für Detektion und Anregung zusammen [91, 92],

$$
U_{\text {con }}(\vec{r})=E(\vec{r}) \cdot D_{\mathrm{eff}}(\vec{r})
$$

$E(\vec{r})$ beschreibt die dreidimensionale Intensitätsverteilung des Anregungslichts. Im Idealfall ist die Verteilung beugungsbegrenzt und hat in der Fokusebene die Form einer Beugungsscheibe nach Gleichung 1.11, wobei $\lambda$ in diesem Fall die Wellenlänge der Anregungslichtquelle ist. Die PSF der Detektion $D_{\text {eff }}(\vec{r})$ ergibt sich aus der Faltung der Blendenfunktion $A_{b}(\vec{r})$ 
Abbildung 2.30: Halbwertsbreite der PSF eines idealen konfokalen Fluoreszenzmikroskops in lateraler Richtung, sowie die Detektionseffizienz für ein punktförmiges Objekt, aufgetragen gegen den Radius der Lochblende. Es wurde angenommen, dass keine StokesVerschiebung auftritt. Die Achsenbeschriftung bezieht sich auf die dimensionslose optische Einheit (siehe Ausdruck 1.10).

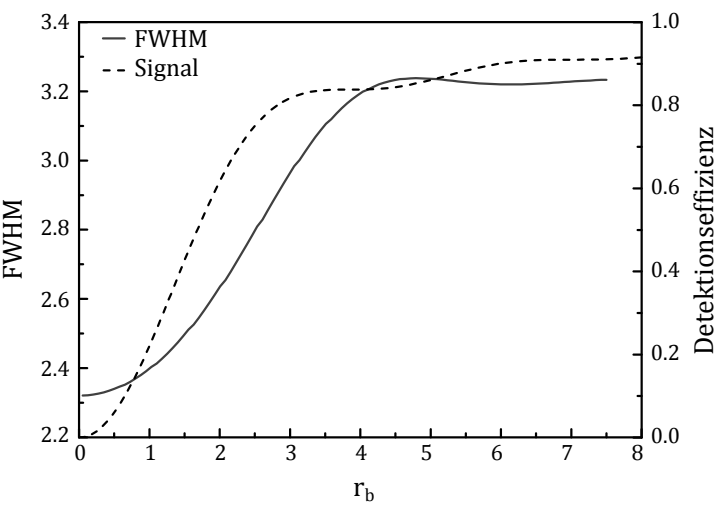

mit der PSF $D(\vec{r})$, eines Weitfeld-Mikroskops, welche ebenfalls durch den Ausdruck 1.11 beschrieben wird,

$$
D_{\text {eff }}(\vec{r})=\left(D * A_{b}\right)(\vec{r}) .
$$

Hierbei repräsentiert der Operator $*$ die Faltungsoperation. Die Blendenfunktion $A_{b}(\vec{r})$ beschreibt die Form der verwendeten Blende projiziert in die Objektebene. Für ein Lochblende mit dem Radius $r_{b}$ in der Objektebene hat $A_{b}$ die Form:

$$
A_{b}(\vec{r})=A_{b}\left(\begin{array}{l}
x \\
y
\end{array}\right)= \begin{cases}1, & \text { wenn } \sqrt{x^{2}+y^{2}} \leq r_{b} \\
0, & \text { sonst }\end{cases}
$$

Mit abnehmendem Durchmesser der Lochblende nimmt die Auflösung des Mikroskops zu, und die Halbwertsbreite der effektiven PSF entsprechend ab. Im Abbildung 2.30 ist die laterale Halbwertsbreite der PSF in der Fokusebene gegen den Durchmesser der Lochblende aufgetragen. Mit zunehmendem Blendendurchmesser nähert sich die PSF eines Konfokalmikroskops derer eines Weitfeld-Mikroskops an. Bei einem sehr kleinen Durchmesser kann die laterale Auflösung, gemessen an der Halbwertsbreite, dagegen bis zu 1.4-fach höher sein. In diesem Fall ist $D_{\text {eff }}=D$, sodass die effektive PSF, unter Vernachlässigung des StokesVerschiebung, dem Quadrat der Weitfeld-PSF entspricht [92].

Mit kleiner werdender Blende nimmt allerdings auch die Gesamtmenge des Lichts ab, das den Detektor erreicht (siehe gestrichelte Kurve in Abbildung 2.30). In der Praxis muss deshalb die Auflösung gegen die Detektionseffizienz abgewogen werden. Üblicherweise wird die Blende so eingestellt, dass deren Radius etwa dem 1-2 fachen des Radius des ersten Minimums der Beugungsscheibe entspricht $\left(r_{b}=3.8-7.6\right.$ in Abbildung 2.30). Die laterale Auflösung ist in diesem Fall kaum größer als die eines Weitfeld-Mikroskop. Die Signalintensität ist jedoch für viele Anwendungen ein limitierender Faktor und der Einsatz von noch kleineren Lochblenden somit nicht sinnvoll [93]. 


\subsubsection{Image-Scanning-Mikroskopie}

Wird die Dichte der Emitter in einer Probe am Punkt $\vec{r}$ durch eine Funktion $S(\vec{r})$ beschrieben, lässt sich die Intensität $I(\vec{r})$ in der mikroskopischen Abbildung über die Faltung von $S(\vec{r})$ mit der PSF $U_{\text {con }}(\vec{r})$ berechnen,

$$
I(\vec{r})=\int U_{\text {con }}\left(\vec{r}-\vec{r}^{\prime}\right) S\left(\vec{r}^{\prime}\right) \mathrm{d} \vec{r}^{\prime}
$$

Wie in Abschnitt 2.2.1.1 beschrieben, gilt für die PSF eines Konfokalmikroskops $U_{\text {con }}(\vec{r})$,

$$
U_{\text {con }}(\vec{r})=\int A_{b}(\vec{s}) D(\vec{r}-\vec{s}) E(\vec{r}) \mathrm{d} \vec{s},
$$

wobei $A_{b}(\vec{s})$ die Blendenfunktion, $E(\vec{r})$ die Intensitätsverteilung des Anregungslichts und $E(\vec{r})$ die Weitfeld-PSF sind. Unter der Annahme eines punktförmigen Detektors (d.h. einer unendlich kleinen Lochblende) vereinfacht sich $U_{\text {con }}(\vec{r}) \mathrm{zu}$

$$
U_{\text {con }}(\vec{r})=D(\vec{r}) E(\vec{r})
$$

Bei der Image-Scanning-Mikroskopie wird der Detektor des Konfokalmikroskops durch einen Flächendetektor ersetzt, sodass für jeden Punkt in der Objektebene ein Bild der Intensitätsverteilung des Fluoreszenzsignals aufgenommen wird. Entsprechend ist die gemessene Intensität sowohl von dem Punkt $\vec{r}$, als auch von der Position $\vec{s}$ auf den Detektor abhängig $[72,75]$. Unter der Annahme eines (unendlich) großen Flächendetektors, der selber nicht als Apertur wirkt, lässt sich der Ausdruck 2.48 somit zu

$$
I(\vec{r}, \vec{s})=\int D\left(\vec{r}-\vec{r}^{\prime}+\vec{s}\right) E\left(\vec{r}-\vec{r}^{\prime}\right) S\left(\vec{r}^{\prime}\right) \mathrm{d} \vec{r}^{\prime}
$$

erweitern. In Abbildung 2.31 wird der Zusammenhang der einzelnen Beiträge zu $I(\vec{r}, \vec{s})$ in einer Dimension illustriert. Hier wird ein fixer Punkt $d_{s}$ auf dem Detektor betrachtet und angenommen, dass ein einzelner Emitter an der Position $r=0$ vorliegt $(S(r)=\delta(r))$. $E(r)$ beschreibt in diesem Fall das Signal des Emitters bei $r=0$ und $D\left(r+d_{s}\right)$ ist die PSF

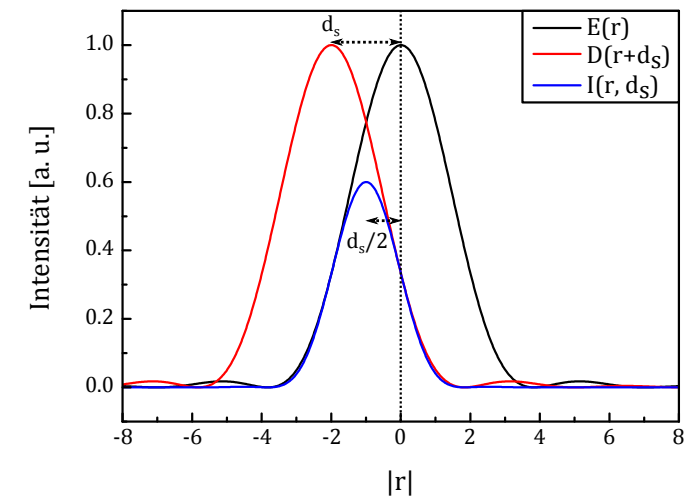

Abbildung 2.31: Illustration der PSF für Detektion $D\left(r+d_{s}\right)$ und Anregung $E(r)$ sowie für die gemessenen Intensitätsverteilung $I\left(r, d_{s}\right)$, für einen Punkt $d_{s}=2$ auf dem Detektor. 
der Detektion für den Punkt $d_{s}=2$ auf dem Detektor. Wird eine Stokes-Verschiebung vernachlässigt, ergibt sich die tatsächlich gemessene Intensität aus dem Produkt der beiden Funktionen und besitzt ein, um $d_{s} / 2$ verschobenes Maximum. Liegt eine Stokes-Verschiebung vor, so ist das Maximum um $\frac{d_{s}}{1+\beta}$ mit $\beta=\frac{\lambda_{e m}}{\lambda_{e x}}$ verschoben [75].

Um ein zweidimensionales Bild aus $I(\vec{r}, \vec{s})$ zu erhalten muss über $s$ integriert werden. Bei direkter Integration über $s$ resultiert das Bild eines Scanning-Mikroskops ohne Lochblende. In diesem Fall entspricht die effektive PSF der Intensitätsverteilung des Anregungslichts. Wird vor der Integration hingegen eine Translation von $\vec{r}$ um $-\vec{s}$ ausgeführt, resultiert das entsprechende Weitfeld-Bild [76]. Ohne Stokes-Verschiebung sind beide Bilder identisch.

Bei der ISM wird die Intensitätsverteilung für jeden Punkt auf dem Detektor nachträglich in das Zentrum der optischen Achse verschoben. Wie Abbildung 2.31 verdeutlicht, muss $\vec{r}$ dazu, vor der Integration um $-\vec{s} / 2$ verschoben werden (bzw. um $\frac{d_{s}}{1+\beta}$ unter Berücksichtigung der Stokes-Verschiebung). Dementsprechend gilt für das ISM Bild

$$
I_{\mathrm{ISM}}(\vec{r})=\int I\left(\vec{r}-\frac{\vec{s}}{2}, \vec{s}\right) d \vec{s}=\iint D\left(\vec{r}-\vec{r}^{\prime}+\frac{\vec{s}}{2}\right) E\left(\vec{r}-\vec{r}^{\prime}-\frac{\vec{s}}{2}\right) S\left(\vec{r}^{\prime}\right) d \vec{r}^{\prime} d \vec{s}
$$

und die PSF von ISM ist damit gegeben durch

$$
U_{\mathrm{ISM}}(\vec{r})=\int D\left(\vec{r}+\frac{\vec{s}}{2}\right) E\left(\vec{r}-\frac{\vec{s}}{2}\right) d \vec{s} .
$$

Durch die Substitution $\vec{r}+\frac{\vec{s}}{2}=2 \vec{s}^{\prime}$ ergibt sich

$$
U_{\text {ISM }}(\vec{r})=4 \int D\left(2 \vec{s}^{\prime}\right) E\left(2 \vec{r}-2 \vec{s}^{\prime}\right) d \vec{s}^{\prime} .
$$

Dem Ausdruck 2.54 lässt sich entnehmen, dass die effektive ISM-PSF aus der Faltung der PSF der Detektion mit der Intensitätsverteilung der Anregung entsteht, jedoch sind beide Funktionen zusätzlich um den Faktor 2 gestaucht [75]. Da die Faltungsoperation im Frequenzraum einer Multiplikation entspricht, ergibt sich für die optische Übertragungsfunktion (OTF) durch Fourier-Transformation

$$
\tilde{U}_{\text {ISM }}(\vec{k})=2 \tilde{D}(\vec{k} / 2) \tilde{E}(\vec{k} / 2),
$$

wobei " $\sim$ " die Fourier-Transformierte der jeweiligen Funktion darstellt [72]. Die OTF ist demnach das Produkt der transformierten Funktionen für Anregung und Detektion, skaliert um den Faktor 2. Das bedeutet die Grenzfrequenz der Übertragungsfunktion ist prinzipiell doppelt so groß wie die eines Weitfeld-Mikroskops. In Abbildung 2.32 ist die Amplitude der lateralen OTF (bzw. die MTF) für ein Weitfeld-Mikroskop, ein ISM sowie für ein Konfokalmikroskop mit unendlich kleiner Apertur aufgetragen. Die Grenzfrequenz eines theoretischen Konfokalmikroskops mit unendlich kleiner Lochblende entspricht ebenfalls der doppelten Frequenz des Abbe-Limits (siehe Ausdruck 1.15), allerdings wäre auch die Detektionseffizienz eines solchen Mikroskops unendlich klein (vgl. Abbildung 2.30). Im Gegensatz dazu 


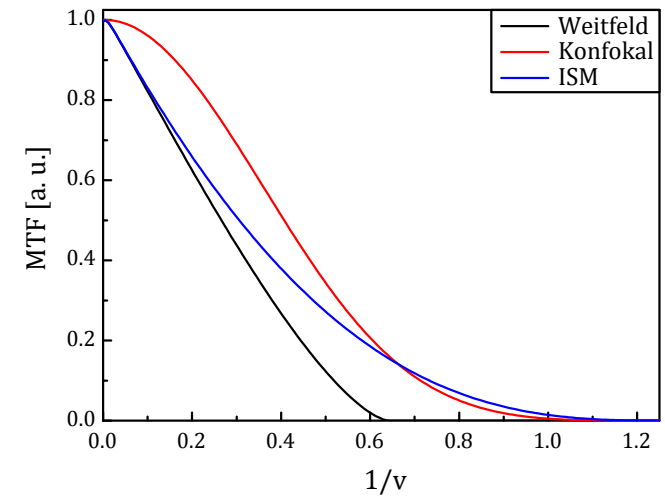

Abbildung 2.32: Auftragung der Modulationsübertragungsfunktion für ein ideales Weitfeld-Mikroskop, ein ISM sowie ein Konfokalmikroskop mit unendlich kleiner Apertur. Die Einheit der Frequenzachse bezieht sich auf die dimensionslose optische Einheit nach Ausdruck 1.10 .

wird durch ein ISM mit ausreichend großem Detektor im Idealfall das gesamte vom Objektiv gesammelte Licht registriert. Gänzlich ohne eine Detektor-Apertur würde dabei jedoch kein optical sectioning erzieht und die Auflösung in axialer Richtung würde der eines WeitfeldMikroskops entsprechen, denn die Stauchung der PSF durch ISM wirkt sich nur in lateraler Richtung aus. Um dennoch das optical sectioning eines Konfokalmikroskops zu erreichen, kann die Fläche der Detektion durch eine Apertur bzw. Blendenfunktion limitiert werden. Die laterale Aufösung eines ISMs ist dabei nur geringfügig von der Größe der DetektorApertur abhängig [75]. Folglich es mit einer geeigneten Apertur prinzipiell möglich eine signifikante Auflösungsverbesserung in lateraler Richtung zu erreichen und gleichzeitig eine axiale Auflösungen beizubehalten, die vergleichbar ist mit der eines Konfokalmikroskops. Beispielsweise ist die laterale Auflösung (FWHM) eines idealen ISMs um das 1.5-Fache höher als die eines Konfokalmikroskops, wenn für beide eine Detektor-Apertur bzw. Lochblende mit dem Radius $r_{b}=3.8$ angenommen wird (vgl. Abbildung 2.30). Die axiale Auflösung und die Detektionseffizienz sind dabei identisch [75].

\subsubsection{Filterung und Dekonvolution von ISM-Daten}

Wie oben erwähnt, ist die Grenzfrequenz der MTF von ISM prinzipiell um den Faktor 2 größer als die eines Weitfeld-Mikroskops. Bedingt durch den flacheren Verlauf der MTF und der resultierenden stärkeren Dämpfung von hohen Frequenzen, ist die Halbwertsbreite der PSF im Vergleich zu einem Weitfeld-Mikroskop aber lediglich um das $\sim 1.5$-fache kleiner. Zur direkten Verdoppelung der Auflösung müsste die effektive PSF der originalen PSF, Skaliert um den Faktor 2, entsprechen. In diesem Fall wäre $U_{\text {eff }}(\vec{r})=D(2 \vec{r})$ und die Transferfunktion $\tilde{U}_{\text {eff }}(\vec{k})=2 \tilde{D}(\vec{k} / 2)$. Enderlein et al. schlagen eine Filterung im Frequenzraum vor, um das ISM-Bild dahingehend zu korrigieren $[72,76]$. Eine passende Filterfunktion wäre prinzipiell

$$
\tilde{W}(\vec{k})=\frac{1}{\tilde{E}(\vec{k} / 2)} .
$$


Hiermit würde Bildrauschen bei hohen Frequenzen allerdings extrem verstärkt werden, da die Filterfunktion bei der Grenzfrequenz von $\tilde{E}(\vec{k} / 2)$ unendlich groß wird. Um dieses Problem zu umgehen, kann ein apodisierter Filter verwendet werden, der zu hohe Frequenzen wiederum dämpft:

$$
\tilde{W}(\vec{k})=\frac{\tilde{D}(\vec{k} / 2)}{\epsilon+\tilde{D}(\vec{k} / 2) \tilde{E}(\vec{k} / 2)} .
$$

Der Parameter $\epsilon$ bestimmt dabei die Grenzfrequenz der Filterfunktion und muss an das Signal-Rausch-Verhältnis der Daten angepasst werden.

In anderen Publikationen wird iterative Dekonvolution für die Rekonstruktion von ISMBildern angewendet [77, 79, 94]. Da hier ebenfalls die Form der PSF berücksichtigt wird, hat die Dekonvolution einen ähnlichen Effekt wie die Filterfunktion von Enderlein et al. Ingaramo et al. haben speziell für ISM Datensätze eine angepasste Dekonvolution nach Richardson und Lucy (RL) vorgeschlagen [95-98], die im Folgenden kurz beschrieben wird: Unter der Annahme, dass auftretende Messfehler ausschließlich auf Poisson-Rauschen zurückzuführen sind, gilt für die Abbildung $\vec{b}$ einer Dichte $\vec{x}$ durch ein optisches System mit der Abbildungsmatrix A,

$$
\vec{b}=\operatorname{Poisson}(\mathbf{A} \vec{x})
$$

Dabei beschreibt die Abbildungsmatrix A die PSF des Abbildungssystems. Die PoissonLikelihood einer Schätzung $\vec{e}$ der Dichte ist somit

$$
L(\vec{e})=\prod \operatorname{Poisson}(\vec{b} \mid \mathbf{A} \vec{e}) .
$$

Bei der RL-Dekonvolution wird $L(\vec{e})$ iterativ maximiert. Folglich nimmt mit jeder Iteration die Wahrscheinlichkeit zu, dass die Schätzung $\vec{e}$ die wahre Dichte wiedergibt. Eine Iteration wird dabei wie folgt durchgeführt:

$$
\begin{aligned}
\vec{b}_{e} & =\mathbf{A} \vec{e}_{i} \\
\vec{r} & =\vec{b} / \vec{b}_{e} \\
\vec{e}_{i+1} & =\vec{e}_{i} \cdot \mathbf{A}^{\top} \vec{r} / \mathbf{A}^{\top} \overrightarrow{1}
\end{aligned}
$$

Hier ist $\vec{e}_{i}$ die Schätzung der realen Dichte $\vec{x}$ bei der $i$-ten Iteration, · und / stehen für punktweise Multiplikation bzw. Division, $\mathbf{A}^{\top}$ ist die Transponierte von $\mathbf{A}$ und $\overrightarrow{1}$ ist ein Vektor bei dem alle Einträge 1 sind.

Der Vektor $\vec{r}$ kann als multiplikativer Korrektur-Faktor aufgefasst werden, mit dem die Schätzung in jeder Iteration modifiziert wird. Gibt $\vec{b}_{e}$ die Messung perfekt wieder, sodass $\vec{b}_{e}=\vec{b}$ ist, werden alle Einträge von $\vec{r}$ eins und $\vec{e}$ ändert sich nicht. Andernfalls werden die Werte in $\vec{e}$ der Abweichung entsprechend angepasst. Durch die Rückprojektion mit der 
transponierten PSF $\mathbf{A}^{\top}$ werden die Korrekturen von $\vec{r}$ dabei an der richtigen Position in $\vec{e}$ angewendet.

Für die RL-Dekonvolution von einem ISM-Datensatz setzt sich $\vec{b}$ aus den Bildern aller Detektorelemente zusammen, hierfür werden die Einträge der einzelnen Bilder gestapelt. Bei Daten mit $n$ Bildpunkten und $m$ Detektorelementen hat $\vec{b}$ folglich $n \cdot m$ Einträge. In gleiche Weise werden in $\mathbf{A}$ die Punktspreizfunktionen für alle Detektorelemente gestapelt, sodass $\mathbf{A}$ eine $n \cdot m \times n$ Matrix und $\mathbf{A}^{\top}$ eine $n \times n \cdot m$ Matrix ist. Die geschätzte Dichte $\vec{e}$ bleibt hingegen ein Vektor mit $n$ Einträgen und die Iterationsschritte der RL-Dekonvolution in Ausdruck 2.60 sind identisch.

\subsubsection{Zeitkorrelierte Einzelphotonenzählung}

Zeitkorrelierte Einzelphotonenzählung (Timecorrelated single photon counting, TCSPC) ist ein messtechnisches Verfahren zur zeitaufgelösten Detektion von Photonen im Piko- und Nanosekundenbereich. Ein Hauptanwendungsgebiet von TCSPC ist die Messung von Fluoreszenzlebensdauern. Detektierte Photonen werden dabei relativ zu einem Synchronisationssignal der gepulsten Anregungslichtquelle erfasst. Aus der Zeitdifferenz zwischen Anregungspuls und Ankunftszeit der Photonen lässt sich ein Histogramm berechnen, dass die Fluoreszenz-Abklingkurve der Probe wiedergibt (siehe Abbildung 2.33) [99]. In klassischer TCSPC-Hardware werden Zeitzu-Amplituden-Umwandler eingesetzt (time-toamplitude converter, TAC), mit denen die Zeitdifferenz zwischen Anregungspuls und Photonenankunftszeit in eine Spannung umgesetzt wird. Die Zeitinformation wird im Anschluss durch einen

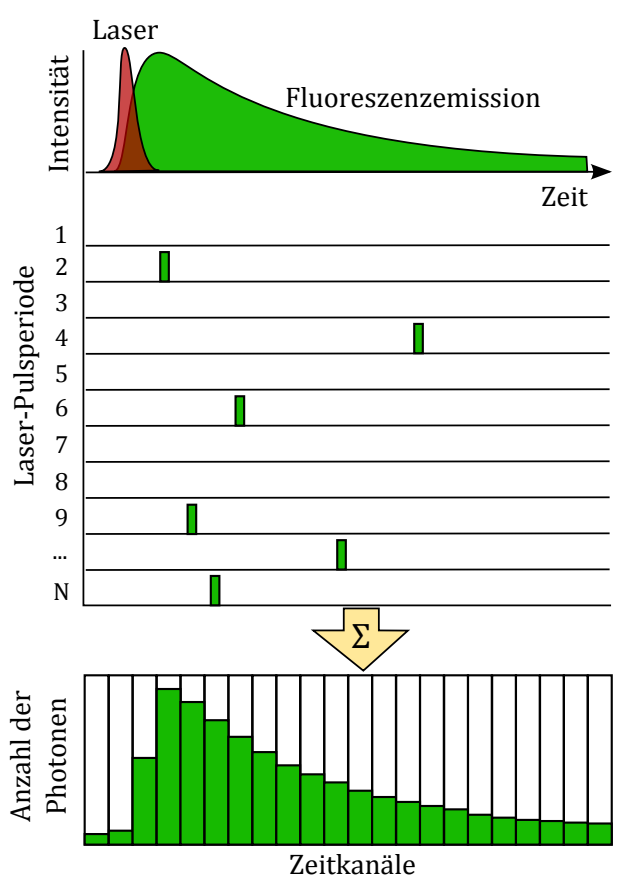

Abbildung 2.33: Prinzip der Messung von Fluoreszenz-Abklingkurven durch zeitkorrelierte Einzelphotonenzählung. A/D-Wandler digitalisiert. Alternativ kann die Ankunftszeit eines Signals mit sog. Time-to-Digital Convertern (TDC) direkt digital erfasst werden. Bei dieser Technik werden Ringoszillatoren mit schnellen digitalen Zählern eingesetzt und für jedes eingehende Signal ein hochauflösender Zeitstempel erzeugt. TCSPCSysteme mit TACs erreichen in der Regel eine höhere Zeitaufösung. Für die Erfassung von 
Fluoreszenzlebensdauern ist die Genauigkeit von modernen TDCs allerdings in den meisten Fällen ausreichend.

\subsubsection{Fluoreszenzlebensdauer}

Die Fluoreszenzlebensdauer ist die mittlere Verweildauer eines Fluorophors im elektronisch angeregten Zustand. Organische Farbstoffe besitzen Fluoreszenzlebensdauern, die typischerweise im Nanosekundenbereich liegen. Zur Depopulation des angeregten Zustands tragen neben der Fluoreszenzemission auch strahlungslose Desaktivierungsprozesse bei (siehe Abschnitt 1.2.2). Die Lebensdauer $\tau$ ist dabei invers proportional zur Summe der Geschwindigkeitskonstanten aller beteiligten Prozesse,

$$
\tau=\frac{1}{\sum_{i} k_{i}} .
$$

Die Fluoreszenz-Abklingkurve eines Fluorophors mit der Lebensdauer $\tau$ wird durch die Exponentialfunktion

$$
I(t)=I_{0} \cdot e^{-\frac{t}{\tau}}
$$

beschrieben.

Bei der Messung von Fluoreszenzlebensdauern durch zeitkorrelierte Einzelphotonenzählung muss berücksichtigt werden, dass die aufgenommene Abklingkurve auch von der Zeitauflösung der Messelektronik sowie der Pulsdauer der Anregungslichtquelle beeinflusst wird. Die gemessene Abklingkurve $I_{m}(t)$ entspricht somit der Faltung des originalen Signals mit der Impuls-Antwort-Funktion (Impulse Response Function, IRF) $U(t)$ des Messsystems,

$$
I_{m}(t)=\left(U * I_{0} \cdot e^{-\frac{t}{\tau}}\right)(t) .
$$

Ist die $U$ bekannt, so kann die Lebensdauer durch Anpassung der Funktion $I_{m}(t)$ an die gemessenen Abklingkurve bestimmt werden. Bei geringer Signalintensität bietet sich hierfür die Maximum-Likelihood-Methoden an. 


\section{$2.3 \quad$ Experimentelles}

\subsubsection{Experimenteller Aufbau}

Bei dem experimentellen Aufbau handelt es sich im wesentlichen um ein beam-scanning CLSM. Als Plattform wird ein modifiziertes Weitfeld-Mikroskop (IX50, Olympus) verwendet, dass mit einen xy-Probentisch (Merzhäuser), sowie einem Piezo-Aktuator in z-Richtung (Custom Nano-Z200, Mad City Labs) ausgestattet ist. Als Anregungslichtquelle wird ein gepulster Diodenlaser (LDH-P-C-485, PicoQuant) mit einer Wellenlänge von $485 \mathrm{~nm}$ und einer maximalen Repetitionsrate von $40 \mathrm{MHz}$ verwendet. Alternativ wird für 2-Photonenanregung ein modengekoppelter Titan:Saphir-Laser (90 MHz Chameleon, Coherent) eingesetzt.

Das Anregungslicht wird zunächst durch ein Linsenpaar aufgeweitet und passiert einen elektronischen Verschlussmechanismus (Compur). Durch einen dichroitischen Spiegel (500DCLP, Omega) wird das Anregungslicht in den Strahlengang des Mikroskops eingebracht. Als Scanner werden ein Galvoscanner (M3, GSI Lumonics) und ein resonanter Scanner (SC-30, Electro-Optical Products Corp.) mit einer Frequenz von 16 kHz verwendet. Der Galvoscan-

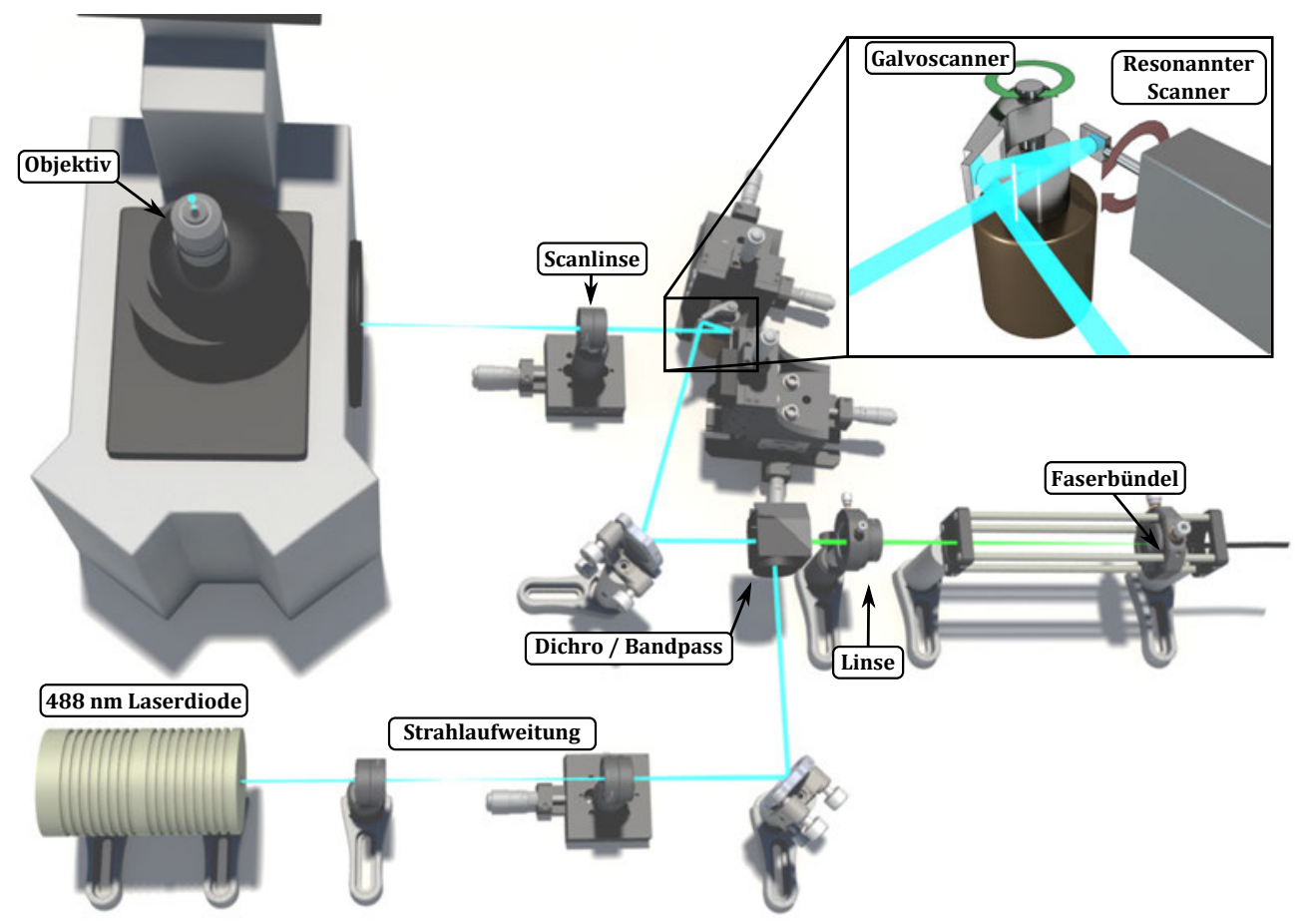

Abbildung 2.34: Aufbau des MDS-Mikroskops. 
Abbildung 2.35: Lichtmikroskopische Aufnahme des Faserbündels. Für das Bild wurde das aufgefächerte Enden des Bündels beleuchtet. Alle 13 verwendeten Fasern sind mit deren Kennnummer markiert. (Maßstab $50 \mu \mathrm{m})$

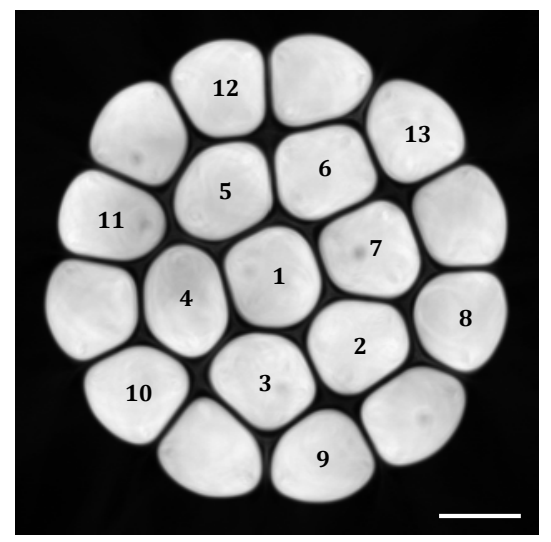

ner steuert den Zeilenvorschub in y-Richtung und der schnellere, resonante Scanner bewegt sich in $\mathrm{x}$-Richtung entlang einer Zeile. Um Relay-Optiken zwischen den Scannern zu vermeiden, wird für den Galvoscanner ein Spiegel verwendet, der abseits der Rotationsachse befestigt ist (off-axis Scanning) [100]. Die beiden Scanner sind so angeordnet, dass kleine Auslenkungen des Galvo-Spiegels keine Translation des Strahlengangs auf dem Spiegel des resonanten Scanners zur Folge haben (siehe Vergrößerung in Abbildung 2.34). Zudem ist der Einfallswinkel auf dem resonanten Scanner möglichst steil, um dessen sehr kleinen Spiegel (4 x $3 \mathrm{~mm}$ ) effizient auszunutzen. Über die Scanlinse (Achromat, $\mathrm{f}=60 \mathrm{~mm}, \varnothing 25.4 \mathrm{~mm}$, Qioptiq) und die Tubuslinse des Mikroskops wird der resonante Scanner-Spiegel auf die Rückapertur des Objektives abgebildet. Dabei handelt es sich um ein Öl-Immersions-Objektiv (UPlanSApo-100x-1.4, Olympus). Das Fluoreszenzlicht wird durch den dichroitischen Spiegel und einen Bandpassfilter (HQ535DF50M, Chroma) von dem Anregungslicht getrennt. Eine Linse (Achromat, $\mathrm{f}=250 \mathrm{~mm}, \varnothing 25.4 \mathrm{~mm}$, Thorlabs) erzeugt schließlich ein Bild des Anregungspunkts auf einem dahinter liegenden Faserbündel mit einer 417-fachen Gesamtvergrößerung. Das Faserbündel selbst ist in einer beweglichen Aufhängung fixiert, sodass sich das Bündel in alle Raumrichtungen justieren lässt.

\subsubsection{Faserbündel und Detektoren}

Der verwendete Detektor setzt sich aus dem Glasfaserbündel sowie einer Reihe von Lawinenphotodioden zusammen. Das Faserbündel wurde von der Firma Ceramoptec angefertigt. Es besteht aus 19 einzelnen Multimode-Fasern mit einem Kerndurchmesser von $60 \mu \mathrm{m}$ und einem Manteldurchmesser von $66 \mu \mathrm{m}$. Wichtig für eine hohe Detektionseffizienz ist hierbei der, im Verhältnis zum Faserkern, kleine Manteldurchmesser. Alle Fasern laufen an einem Ende in einer hexagonalen Anordnung zusammen (siehe Abbildung 2.35). Um eine höher Packungsdichte zu erreichen, wurden die Fasern durch Erhitzen verschmolzen [101], was al- 


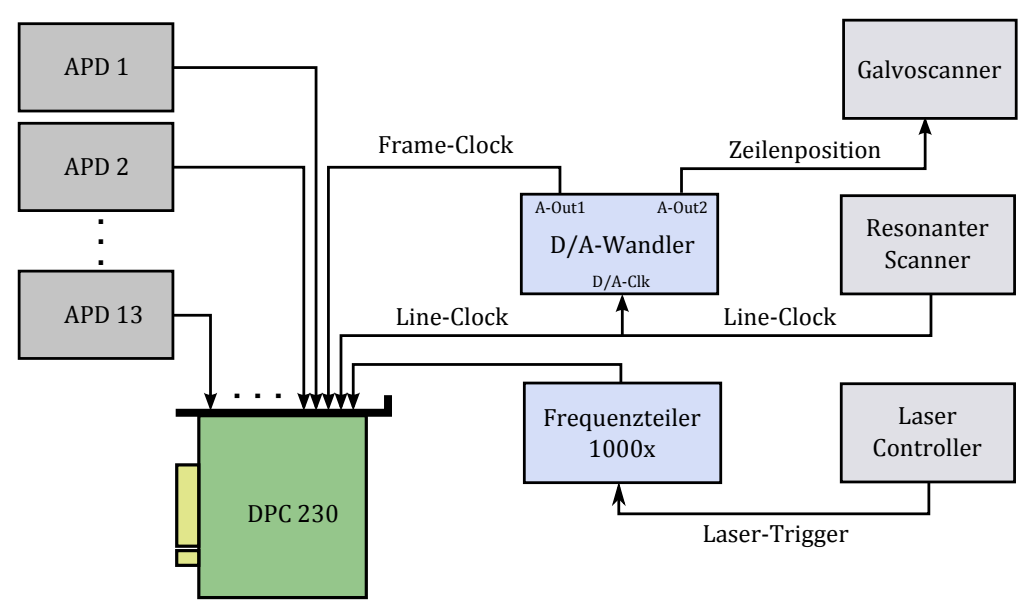

Abbildung 2.36: Elektronische Komponenten des Versuchsaufbaus.

lerdings eine leichte Verformung der Kerne verursacht hat. Die verwendete Messelektronik erlaubt die gleichzeitige Aufnahme in 16 Kanälen, von denen drei für Zeitsignale von den Scannern sowie dem Laser verwendet werden. Aus diesem Grund konnten effektiv nur 13 der 19 Fasern für die Datenaufnahme genutzt werden. An dem aufgefächerten Ende des Bündels ist jede dieser 13 Fasern mit einer Einzelphotonen-Lawinenphotodiode (SPAD-AQR-13-FC, PerkinElmer) verbunden. Die Detektoren besitzen eine Zeitauflösung von $\sim 500 \mathrm{ps}^{(11)}$ und eine Quanteneffizienz von $55 \%$ im Detektionsfenster von $510 \mathrm{~nm}$ bis $560 \mathrm{~nm}$. Die Dunkelzählrate beträgt abhängig von der Diode zwischen $100 \mathrm{~Hz}$ und $250 \mathrm{~Hz}$.

\subsubsection{Elektronik und Messtechnik}

Für die Datenerfassung wird eine Messkarte zur zeitkorrelierten Einzelphotonenzählung mit 16 digitalen Eingängen verwendet (DPC-230, Becker \& Hickl). Die 16 Kanäle der Karte können parallel TTL-Signale mit einer Zeitauflösung von 160 ps erfassen. Neben den Signalen der 13 SPADs werden Taktsignale für Zeilenwechsel, Bildwechsel und die Laserpulse erfasst (siehe Abbildung 2.36). Die Auslenkung des Galvoscanners wird über ein Analogsignal gesteuert, dass durch einen computergesteuerten Digital-Analog-Wandler (USB-1608GX-2AO, Measurement Computing) erzeugt wird. Der Takt des D/A-Wandlers wird dabei von dem resonanten Scanner vorgegeben, der für jede Schwingungsperiode des Spiegels ein TTL-Puls ausgibt. Das Steuersignal für den Galvoscanner hat die Form einer gestuften Sägezahnschwingung, wobei die Rückflanke eine endliche Steigung besitzt, um ein Überschwingen des Galvospiegels zu minimieren. Am Ende jeder kompletten Schwingungsperiode des resonanten Scanners findet ein Zeilenwechsel statt. Unter Berücksichtigung der Rücklaufzeit des

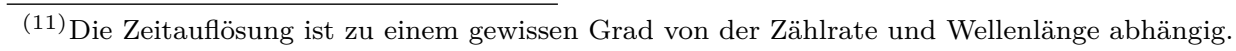


Galvoscanners, ergibt sich bei der Zeilenfrequenz von $16 \mathrm{kHz}$ eine maximale Aufnahmegeschwindigkeit von $28 \mathrm{~Hz}$ für ein Bild mit 512 Zeilen. Um den Anfang des Bildes zu markieren, wird der Startpunkt der Sägezahnkurve durch einen TTL-Puls von dem D/A-Wandler an die Messkarte übertragen. Außerdem wird ein Taktsignal der gepulsten Lichtquelle erfasst, um ggf. Fluoreszenz-Abklingkurven berechnen zu können. Da die Repetitionsrate der Laser (40 MHz oder $90 \mathrm{MHz}$ ) die maximale Zählrate der Messkarte von $\sim 8 \mathrm{MHz}$ weit überschreitet, wird ein Frequenzteiler verwendet, der nur jeden tausendsten Puls an die Messkarte weitergibt. Die Frequenzstabilität des Lasers ist ausreichend, um das resultierende $40 \mathrm{kHz}$ Signal zur Berechnung der Fluoreszenz-Abklingkurven zu nutzen.

\subsubsection{Datenerfassungssoftware}

Da die vom Hersteller der TCSPC-Messkarte vertriebene Software nicht den Anforderungen des Experiments entsprach, wurde eine eigene Softwarelösung für die Datenaufnahme entwickelt. Als Plattform wurde dabei die quelloffene Mikroskopie-Software Micro-Manager [102] verwendet, die mit selbst entwickelten Geräte-Adaptern für den experimentellen Aufbau erweitert wurde. Sowohl die Datenaufnahme, als auch die Steuerung der Galvoscanner wurden über Micro-Manager Geräte-Adapter realisiert, die in den Programmiersprachen C und $\mathrm{C}++$ implementiert wurden.

Die Datenerfassung wird Hardware-intern durch zwei separate sog. Time-to-Digital-Converter (TDC) Bausteine realisiert, die den Zeitpunkt und Kanal eines eintreffenden Signals, z.B eines detektierten Photons, aufzeichnen. Der Rohdatenstrohm aus den Puffern der beiden TDC-Bausteine muss deshalb zunächst aufgearbeitet und in der richtigen zeitlichen Abfolge zusammengeführt werden ${ }^{(12)}$. Danach wird aus den Ankunftszeiten der einzelnen Photonen, mit Hilfe der Synchronisationssignale für Bild- und Zeilenanfang, eine Position im Bildkoordinatensystem berechnet. Hierbei muss berücksichtigt werden, dass der Zusammenhang zwischen Zeit und Position des resonanten Scanners nicht linear ist, sondern aufgrund der harmonischen Schwingung einer Kosinusfunktion entspricht. An den Umkehrpunkten des Scanners ist die Verweildauer und damit die Nichtlinearität am größten. Folglich müssen diese Bereiche von der Bilderfassung ausgeschlossen werden, damit die Pixelintegrationszeit im Bild ausreichend homogen bleibt. Im Experiment werden etwa $80 \%$ der Periodendauer des resonanten Scanners für den Bildaufbau genutzt. Im konfokalen Modus des Mikroskops wird die Intensität an der berechneten Position im Bild für jedes detektierte Photon in-

\footnotetext{
(12) Als Vorlage für die Implementierung dieses Verarbeitungsschrittes wurde Programmcode verwendet, der freundlicherweise von Dr. Marcel Leutenegger (MPI für biophysikalische Chemie) zur Verfügung gestellt wurde.
} 
krementiert, unabhängig von welchem Detektor das Photon registriert wurde ${ }^{(13)}$. Im MDSModus wird zu der Position ein Offset addiert, der von der jeweiligen Position der Faser abhängt. Parallel zu der Bilderzeugung kann der Datenstrom für eine spätere Auswertung auf die Festplatte geschrieben werden. Um die Umwandlung des Rohdatenstroms und die Datenaufnahme zeitgleich durchzuführen, werden die verschiedenen Schritte der Datenverarbeitung in mehreren parallel laufenden Threads bearbeitet. Auf diesem Weg können sowohl konfokale als auch MDS-Bilder bis zu der maximalen Zählrate der Messkarte $(\sim 8 \mathrm{MHz})$ in Echtzeit ausgegeben werden.

\subsubsection{Registrierung von MDS-Rohdaten}

Im MDS-Modus des Mikroskops kann für jede Faser prinzipiell ein separates Bild erfasst werden. Die einzelnen Bilder weisen eine laterale Verschiebung auf, die von der Position der jeweiligen Faser abhängig ist. Unterscheiden sich zwei Bilder des gleichen Objektes lediglich durch eine konstante Translation, so kann der Translationsvektor über eine normalisierte Kreuzkorrelation ermitteln werden [103, 104]. Diese Methode wird bei der Auswertung der MDS-Rohdaten verwendet, um die Translation der einzelnen Bilder relativ zu dem Bild der zentralen Faser zu bestimmen. Durch eine entgegengesetzte Transformation wird die Verschiebung kompensiert und das MDS-Bild aus der Summe aller so registrierten Bilder berechnet. Das hierfür verwendete Matlab-Skript ist in Anhang A.2 aufgeführt. Wenn die Translation für die einzelnen Detektoren durch eine vorangegangene Messung bekannt ist, kann diese schon während der Bildaufnahme berücksichtigt werden.

\subsubsection{Dekonvolution}

Dekonvolutionen wurden, wie in Abschnitt 2.2.2.1 beschriebenen, mit einem für MDS-Daten angepassten Algorithmus nach Richardson und Lucy durchgeführt [95, 96]. Aus Effizienzgründen wurde die Punktspreizfunktionen dabei nicht durch eine vollbesetzte Abbildungsmatrix dargestellt, sondern eine Faltung im Fourierraum durchgeführt [105]. Um die Entstehung von Artefakten auszuschließen, wurden der Algorithmus schon nach 3 Iterationen abgebrochen. Als PSF wurden die gemessenen Punktspreizfunktionen der einzelnen Fasern verwendet. Der Algorithmus wurde in der Programmiersprache Python implementiert [106]. Das entsprechende Skript ist in Anhang A.4 aufgeführt.

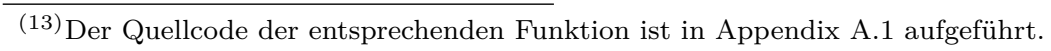




\subsubsection{Fluoreszenzlebensdauer-Mikroskopie}

Für die Berechnung von Fluoreszenzlebensdauer-Mikroskopie-Bildern wurden die Rohdaten zunächst mit Matlab verarbeitet und anschließend mit der Software FLIMfit ausgewertet [107]. Die Impuls-Antwort-Funktion für die Auswertung wurde aus der monoexponentiellen Fluoreszenz-Abklingkurve des Farbstoffs Oregon Green durch RL-Dekonvolution gewonnen.

\subsubsection{Justage des Faserbündels}

Um eine möglichst große Detektionseffizienz zu erreichen, muss das Ende des Faserbündels in der Bildebene und dessen Mittelpunkt exakt auf der optischen Achse liegen. Für die entsprechende Justage wurde ein Objektärger benutzt, auf dem sich dünner Film aus Farbstoffösung (Oregon Green 488, Life Technologies) befand. Im Justagemodus der Messsoftware werden die Zählraten aller Detektoren kontinuierlich ausgelesen und durch eine 2D-Gauß-Anpassung laufend Schwerpunkt und Halbwertsbreite der Intensitätsverteilung bestimmt. Auf diesem Weg konnte die Position des Faserbündels exakt eingestellt werden.

\subsubsection{Proben}

\subsubsection{Microspheres}

Eine verdünnte Suspension von Microspheres in $\mathrm{ddH}_{2} \mathrm{O}(0.2 \mathrm{mg} / \mathrm{mL}$, FS02F-PS(480,520) $0.051 \mu \mathrm{m}$, Bangs Laboratories) wurde auf ein mit Isopropanol und $\mathrm{dd}_{2} \mathrm{O}$ gereinigtes Deckgläschen gegeben. Die Suspension wurde sofort wieder entfernt und die Dichte der Microspheres auf der Glasoberfläche unter dem Mikroskop geprüft. Der Vorgang wurde gegebenenfalls wiederholt bis sich etwa 10-20 Microspheres im Bildausschnitt befanden. Anschließend wurde das Deckgläschen mit $\mathrm{ddH}_{2} \mathrm{O}$ benetzt und die Probe vermessen.

\subsubsection{Fixierte BPAE-Zellen}

Präparate mit fixierten BPAE-Zellen (FluoCells Prepared Slide \#2) wurden von der Firma Life Technologies GmbH bezogen. Die Mikrotubuli der Zellen sind immunologisch mit dem Fluoreszenzfarbstoff BODIPY FL (Abs. 503 nm, Em. $512 \mathrm{~nm}$ ) und Aktinfilamente mit dem Farbstoff Texas Red-X Phalloidin (Abs. 591 nm, Em. 608 nm) markiert. Zudem ist der Zellkern mit dem DNA bindenden Farbstoff DAPI (Abs. 358 nm, Em. 461 nm) gefärbt. 


\subsection{Ergebnisse und Diskussion}

\subsubsection{Theoretische Auflösung}

Bei der theoretischen Betrachtung der Image-Scanning-Mikroskopie in Kapitel 2.2.2 wurde angenommen, dass der Detektor kontinuierlich ist und folglich aus unendlich vielen Detektorelementen besteht. Im Experiment werden jedoch nur 13 Fasern verwendet, die zudem unregelmäßig angeordnet sind. Um die theoretisch erreichbare Auflösung für den experimentellen Aufbau abzuschätzen, müssen die Position und Form der einzelnen Fasern berücksichtigt werden. Jede Faser kann dabei als Aperturblende eines separaten konfokalen Mikroskops betrachtet werden. Wie in Abschnitt 2.2.1.1 erläutert wird, kann die effektive PSF eines konfokalen Mikroskops berechnet werden, indem die Blendenfunktion mit der PSF für die Detektion $D(\vec{r})$ gefaltet und anschließend mit der Intensitätsverteilung der Anregung $E(\vec{r})$ multipliziert wird. Die Blendenfunktion einer Faser lässt sich aus der Aufnahme des Bündelendes bei Beleuchtung des Detektorendes abschätzen (siehe 2.35). Dabei wird angenommen, dass die gemessene Intensität in etwa proportional zu der ortsabhängigen Detektionseffizienz ist. In Abbildung 2.37b ist die resultierende PSF für die zwei in 2.37a markierten Fasern dargestellt. Wobei für $D(\vec{r})$ und $E(\vec{r})$ eine ideale Beugungsscheibe nach Gleichung 1.11 angenommen wurde. Das Maximum der Intensitätsverteilung der Anregung $E(\vec{r})$ befindet sich auf der optischen Achse im Zentrum des Bündels. Folglich haben die Punktspreizfunktionen ihre Maxima etwa auf halber Strecke zwischen dem Schwerpunkt der jeweiligen Faser und dem Mittelpunkt des Bündels. Die effektive PSF im klassischen konfokalen Betrieb entspricht dabei der Summe der Punktspreizfunktionen für alle verwendeten Fasern. Für die effektive Punktspreizfunktion im MDS-Modus muss die PSF jeder Faser zuvor auf die optische Achse verschobenen werden. In Abbildung 2.37c ist die resultierende MDS-PSF für zwei und in 2.37e für alle 13 Fasern dargestellt. Die Berechnungen zeigen, dass die leichten Verformungen der Fasern, die durch das Verschmelzen des Bündels entstanden sind, kaum Einfluss auf die PSF haben. Auch die etwas unregelmäßige Anordnung der Fasern untereinander verursacht keine signifikante Verzerrung der PSF.

Bei einem herkömmlichen Konfokalmikroskop ist die Größe der Lochblende ausschlaggebend für dessen theoretische Aufösung. Im Experiment kann die effektive Größe der Faser (bzw. deren Projektion in die Objektebene) nur über die Vergrößerung des Abbildungssystems verändert werden. Dabei stellt sich die Frage, bei welcher Vergrößerung die Auflösungsverbesserung durch MDS, unter Berücksichtigung des verwendeten Faserbündels, am größten ist. Um diese Frage zu beantworten, wurden die Berechnungen für Abbildung 2.37 


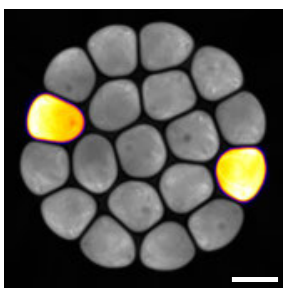

(a)

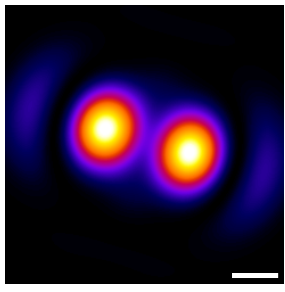

(b)

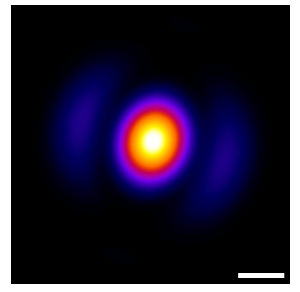

(c)

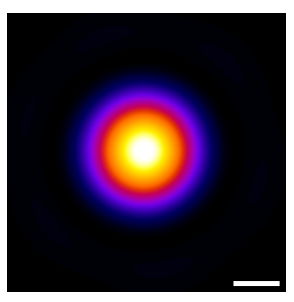

(d) PSF konfokal

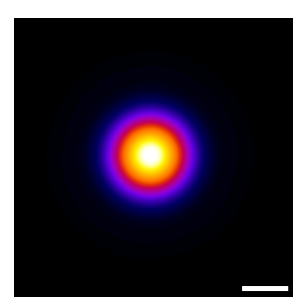

(e) PSF MDS

Abbildung 2.37: Theoretische Punktspreizfunktion. a: Lichtmikroskopische Aufnahme des Faserbündels. Zwei Fasern sind farblich hervorgehoben. b: Konfokale PSF für die beiden in a hervorgehobenen Fasern. c: PSF der beiden Fasern im MDS-Modus. d: PSF für alle 13 Fasern in konfokalen Modus. e: PSF für alle 13 Fasern im MDS Modus. Für die Brechungen wurden folgende Parameter angenommen: $\lambda_{e x}=488 \mathrm{~nm}, \lambda_{e m}=525 \mathrm{~nm}, \mathrm{NA}=1.4$, Vergrößerung: 500 -fach. Die Helligkeit der Bilder wurde normalisiert. (Maßstab: $50 \mu \mathrm{m} / 100 \mathrm{~nm}$ )

Abbildung 2.38: Theoretische Halbwertsbreite der PSF aufgetragen gegen die optische Vergrößerung.

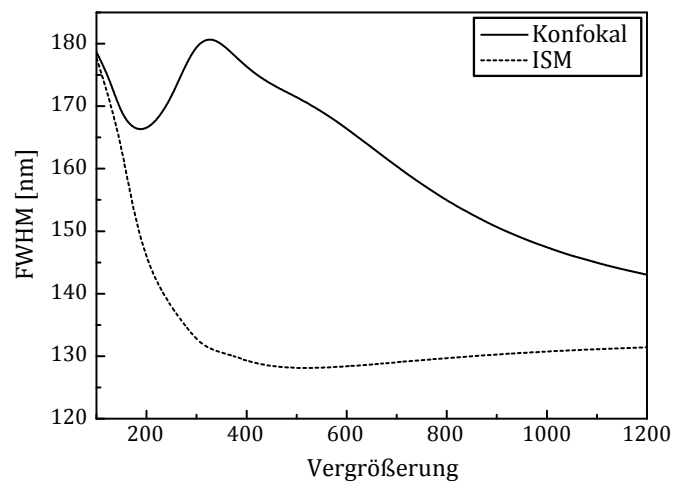

bei unterschiedlichen Vergrößerungen durchgeführt und die Halbwertsbreite der PSF im konfokalen sowie im MDS-Modus bestimmt. Die Punktspreizfunktionen der Anregung und Detektion wurden hierfür nach Gl. 1.11 mit den zum Versuchsaufbau passenden Parametern $\left(\lambda_{e x}=488 \mathrm{~nm}, \lambda_{e m}=525 \mathrm{~nm}, \mathrm{NA}=1.4\right)$ berechnet. Wie die entsprechende Auftragung in Abbildung 2.38 zeigt, wirkt das Faserbündel bei kleiner Vergrößerung als Apertur mit großem Durchmesser. Die laterale Auflösung nähert sich dabei für beide Modi der Auflösung eines Scanning-Mikroskops mit geöffneter Lochblende an. Umgekehrt strebt die Halbwertsbreite mit zunehmender Vergrößerung gegen die eines CLSM mit unendlich kleiner Lochblende. Für MDS nimmt die Halbwertsbreite dabei erwartungsgemäß schneller ab. Die relative Auf- 
lösungsverbesserung durch MDS erreicht ihr Maximum bei 350-facher Vergrößerung, wobei die Halbwertsbreite um den Faktor 1.38 kleiner, als im konfokalen Modus ist. Das Minimum der Kurve befindet sich mit $128 \mathrm{~nm}$ bei 525-facher Vergrößerung.

Im experimentellen Aufbau wurde ein Linsensystem mit einer fixen Vergrößerung von 417 verwendet. Die konfokale PSF hat bei diesem Wert eine Halbwertsbreite von $175 \mathrm{~nm}$ und die MDS-PSF eine Halbwertsbreite von $129 \mathrm{~nm}$. Theoretische lässt sich die Auflösung durch MDS im experimentellen Aufbau danach um den Faktor 1.36 verbessern. Allerdings wird hier von einem idealen Abbildungssystem ausgegangen, Abweichungen im Brechungsindex des Einbettungsmediums sowie Imperfektionen im Abbildungssystem führen unter realen Bedingungen zu Aberrationen und somit schlechteren Ergebnissen [108, 109]. Des weiteren wird hier keine zusätzliche Auflösungsverbesserung durch Filterung oder Dekovolution berücksichtigt.

Grundsätzlich ist die erreichbare Auflösungsverbesserung von der Anzahl der Detektorelemente abhängig. Werden alle 19 Fasern des Bündels in die Berechnung einbezogen, steigt der Faktor von 1.38 auf 1.42. Der Maximalwert bei $\lambda_{e x}=488 \mathrm{~nm}$ und $\lambda_{e m}=525 \mathrm{~nm}$ für ein ISM mit kontinuierlichem Flächendetektor beträgt 1.47. Folglich kann die Auflösungsverbesserung durch zusätzliche Detektoren noch gesteigert werden, wobei der relative Zugewinn mit steigender Anzahl jedoch sinkt. Abgesehen von praktischen Aspekten wird die sinnvolle Anzahl an Detektoren vor allem durch deren Dunkelzählrate limitiert. Bei gleichbleibender Vergrößerung kann die Detektionseffizienz durch zusätzliche Detektoren prinzipiell gesteigert werden, im Gegenzug nimmt jedoch auch das Hintergrundsignal durch die Dunkelzählrate zu, wodurch das Signal-Rausch-Verhältnis ab einem gewissen Punkt wieder kleiner wird.

\subsubsection{Reale Punktspreizfunktion}

Für die Charakterisierung der realen Punktspreizfunktion des Versuchsaufbaus wurden fluoreszierende Polystyrol-Microspheres vermessen. Mit einem Durchmesser von $50 \mathrm{~nm}$ sind die verwendeten Microspheres deutlich kleiner, als die Auflösungsgrenze und können somit in guter Näherung als Punktemitter angesehen werden. Das gemessene Intensitätsprofiel entspricht folglich in guter Näherung der PSF des Versuchsaufbaus [63].

Für die Messungen wurde ein Z-Stapel der Probe mit einer axialen Schrittweite von $150 \mathrm{~nm}$ und einer Pixelgröße von $33 \mathrm{~nm}$ aufgenommen. Die PSF wurde aus der Summe von 13 Microspheres berechnet, die zuvor durch Kreuzkorrelation [104] registriert wurden ${ }^{(14)}$. In Abbildung 2.39 ist die resultierende PSF in der Fokusebene für jede Faser separat abgebil-

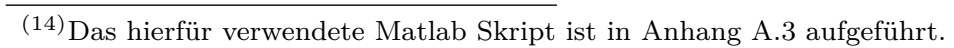



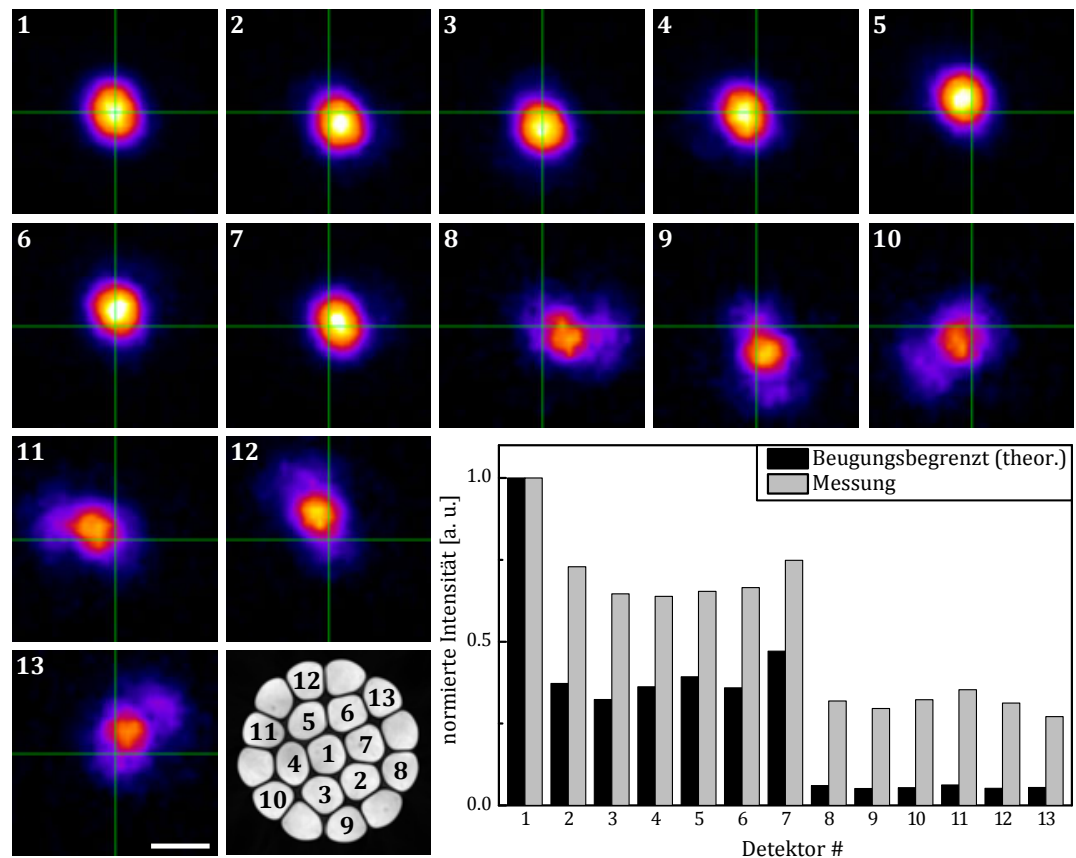

13

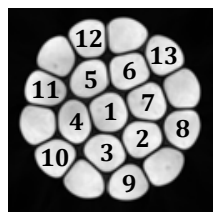

Detektor \#

Abbildung 2.39: PSF der einzelnen Fasern aus einer Messung von Microspheres. Abgebildet ist der xy-Schnitt durch die Fokusebene. Die Helligkeiten wurde normiert. Die Auftragung zeigt die detektierte Gesamtintensität für die einzelnen Fasern im Vergleich mit der theoretisch erwarteten Intensitätsverteilung. (Maßstab: $250 \mu \mathrm{m}$ )

det. In Übereinstimmung mit den theoretischen Berechnungen ist hier eine Translation der Intensitätsmaxima, abhängig von den Positionen der Fasern, zu erkennen. Im Vergleich mit den theoretischen Berechnungen in Abschnitt 2.4.1 sind die Punktspreizfunktionen allerdings größer als erwartet, wobei die Halbwertsbreite für die Fasern 1-7 $\sim 175 \mathrm{~nm}$, gegenüber einem theoretischen Wert von $\sim 130 \mathrm{~nm}$, beträgt. Die Auftragung in Abbildung 2.39 zeigt zudem, dass die detektierte Intensität für die äußeren Fasern deutlich größer ist, als für eine beugungsbegrenzte Verteilung des Anregungslichts unter idealen Bedingungen zu erwarten wäre. Dieses deutet auf einige Imperfektionen im Abbildungssystem hin, die eine Vergrößerung des Anregungspunkts zur Folge haben.

Die effektive 3D-PSF im konfokalen Modus wurde aus der Summe der Punktspreizfunktionen aller Fasern berechnet und ist in Abbildung 2.40 dargestellt. Für die MDS-PSF wurden die einzelnen Punktspreizfunktionen der Fasern registriert und anschließend summiert.

Vor allem die konfokale PSF ist in der xy-Ebene asymmetrisch, wobei eine genauere Betrachtung der Daten zeigt, dass die Ausrichtung der Verzerrung von z-Position abhängt. Demzufolge liegt ein leichter axialer Astigmatismus im Abbildungssystem vor, der wahrscheinlich 


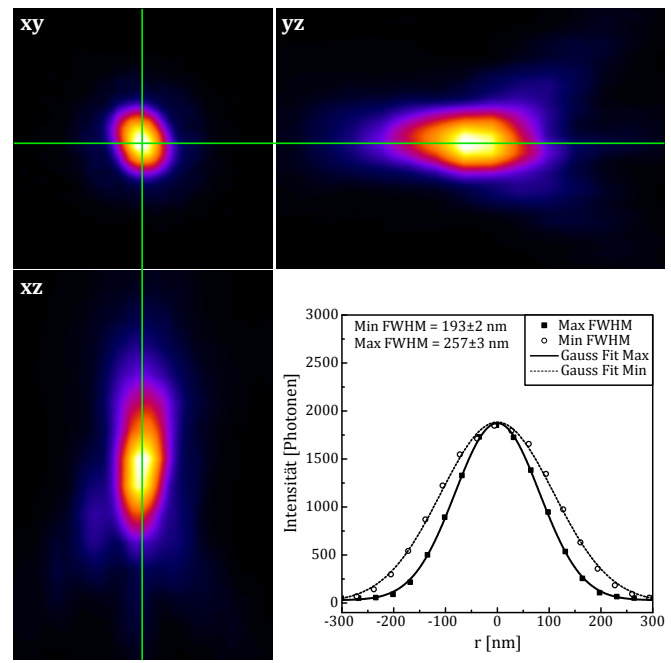

(a) Konfokal

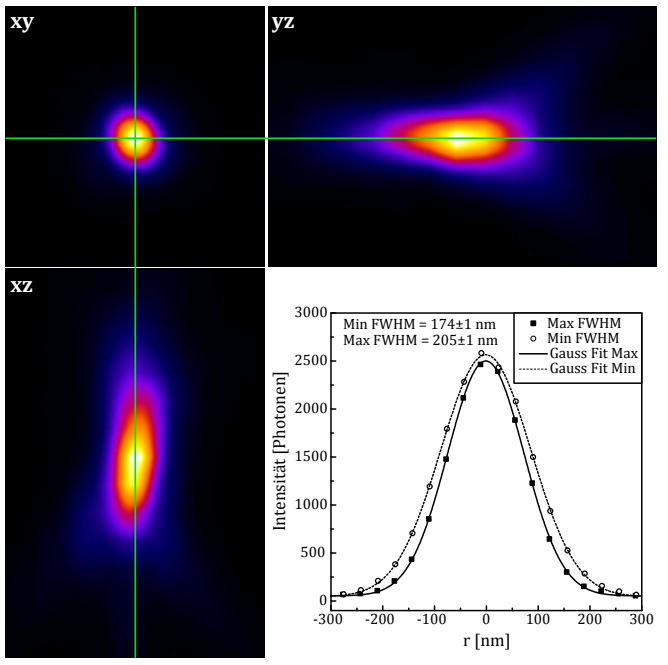

(b) MDS

Abbildung 2.40: 2D-Sektionen der 3D-PSF aus der Messung von Polystyrol-Microspheres im konfokalen (a) und MDS-Modus (b). Die Auftragung zeigt das Intensitätsprofil in der xy-Ebene bei maximaler und minimaler lateraler Ausdehnung der PSF sowie eine entsprechende GaußAnpassung.

durch eine Verformung eines Scanner- oder dichroitischen Spiegels verursacht wird [110]. Aus diesem Grund werden im Folgenden jeweils die maximalen und minimalen Halbwertsbreiten der Punktspreizfunktionen verglichen. Am schmalsten Punkt wiest die konfokale PSF eine Halbwertsbreite von $(193 \pm 2) \mathrm{nm}$, die PSF mit MDS dagegen eine von $(174 \pm 1) \mathrm{nm}$ auf. Das entspricht einer 1.11-fachen Verbesserung der Aufösung durch MDS. Die maximale Halbwertsbreite der konfokalen PSF beträgt $(257 \pm 3) \mathrm{nm}$ und (205 \pm 1$) \mathrm{nm}$ im MDS-Modus und ist damit um den Faktor 1.25 kleiner. Folglich ist die laterale Auflösung des Versuchsaufbaus im MDS-Modus generell besser, als im konfokalen Modus. Bedingt durch die angesprochenen Abbildungsfehler ist die Verbesserung allerdings signifikant kleiner, als der theoretische Wert von 1.36 .

Die axiale Auflösung ist für beide Modi erwartungsgemäß ähnlich und beträgt $\sim 535 \mathrm{~nm}$.

\subsubsection{Biologische Proben}

Zur Untersuchung der tatsächlich erreichbaren Auflösung mit biologischen Proben wurden fixierte BPAE (bovine pulmonary artery endothelial) Zellen vermessen [111]. Dabei handelt es sich um eine etablierte Ziellinie von Endothelzellen der Lungenarterie eines Rindes [112, $113]$. 


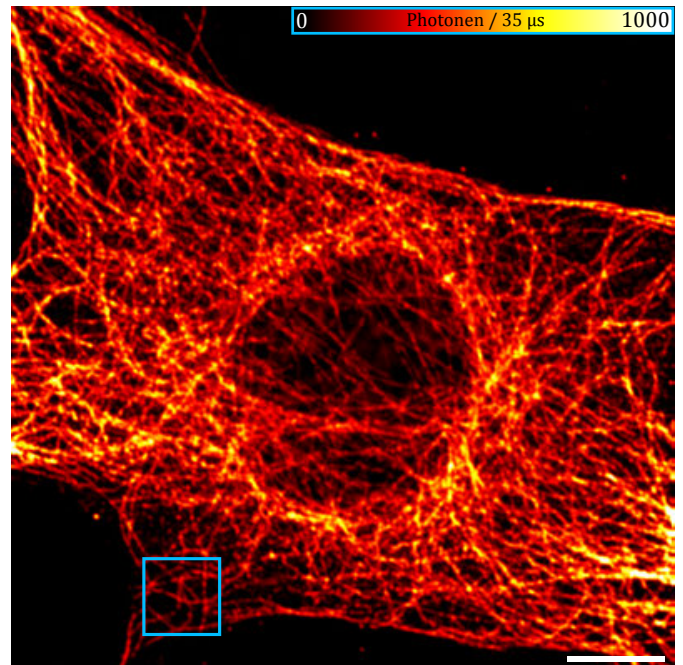

(a) Konfokal

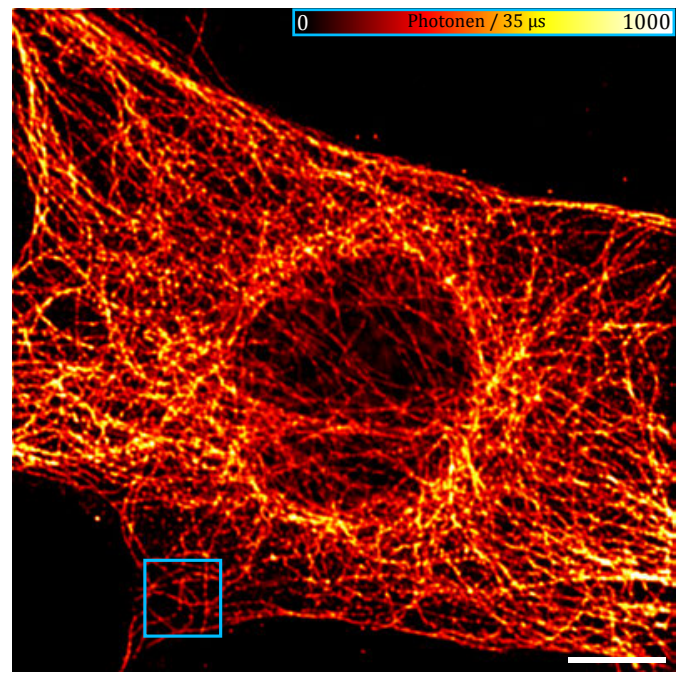

(c) MDS mit Dekonvolution

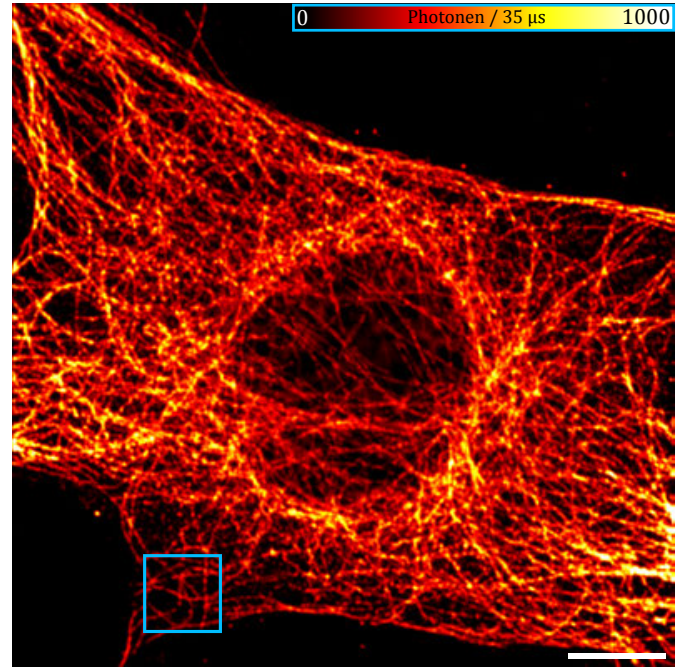

(b) MDS
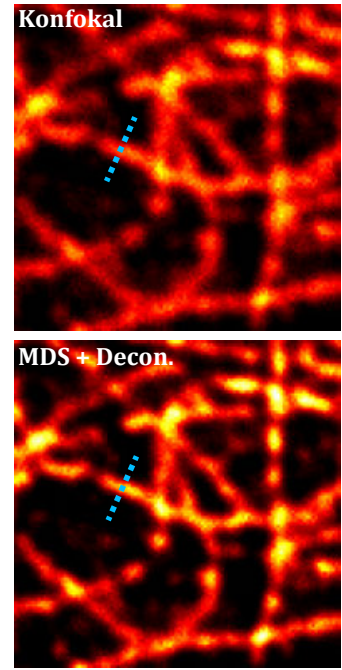

(d) Intensitätsprofil
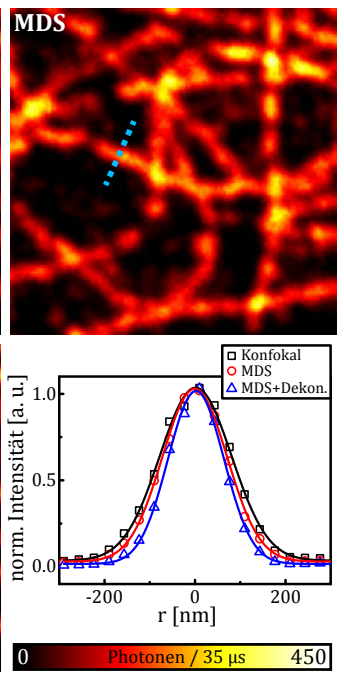

Abbildung 2.41: BODIPY FL-markierte Mikrotubuli von fixierten BPAE-Zellen. Die Aufnahme besteht aus $1024 \times 1024$ Bildpunkten mit einer Größe von $33 \mathrm{~nm}$. Die Integrationszeit pro Bildpunkt betrug durchschnittlich $35 \mu \mathrm{s}$. (Anregungsleistung $8 \mu \mathrm{W}$ bei $485 \mathrm{~nm}$ ) a: Konfokales Bild berechnet aus der Summe aller Detektoren. b: MDS Bild. c: RL-Dekonvolution des MDS-Bildes nach drei Iterationen. d: Vergrößerung des markierten Bereichs $(3.7 \mu \mathrm{m} \times 3.7 \mu \mathrm{m})$ und eine Auftragung der Intensitätsprofile entlang der Markierung mit Gauß-Anpassung. Die Halbwertsbreiten betragen: Konfokal $(187 \pm 4)$ nm, MDS $(168 \pm 3)$ nm, MDS mit Dekonvolution $(146 \pm 3) \mathrm{nm}$. (Maßstab: $5 \mu \mathrm{m})$

Die Abbildung 2.41 zeigt BODIPY FL-markierte Mikrotubuli der Zellen, die mit einer Wellenlänge von $485 \mathrm{~nm}$ angeregt wurden. Für das MDS-Bild wurden die Daten der einzelnen Detektoren untereinander durch Kreuzkorrelation registriert und addiert. Wie zuvor, ent- 
spricht das konfokale Bild der Summe über alle Detektoren. Des weiteren ist in Abbildung 2.41c die RL-Dekonvolution der MDS-Daten dargestellt.

Der Vergleich zwischen konfokalen und MDS-Bild zeigt eine moderate Verbesserung der Auflösung durch MDS. Die Halbwertsbreiten der in Abbildung dargestellten Intensitätsprofile eines einzelnen Filaments betragen $(187 \pm 4) \mathrm{nm}$ im konfokalen und (168 \pm 3$) \mathrm{im}$ MDS-Modus und sind damit sogar noch etwas höher als die minimalen Werte für die experimentell ermittelten Punktspreizfunktionen in Abschnitt 2.4.2. Durch Dekonvolution konnte die Auflösung nochmals erhöht und eine Halbwertsbreite von (146 \pm 3$)$ nm erreicht werden. Das entspricht einer Verbesserung um den Faktor 1.11 für MDS ohne und 1.28 mit Dekonvolution.

Diese Werte sind signifikant kleiner als theoretisch erwartet. Dennoch ist die erreichte Auflösung vergleichbar mit der anderer ISM-Methoden. Schulz et al. geben für ihrer CSD-Variante

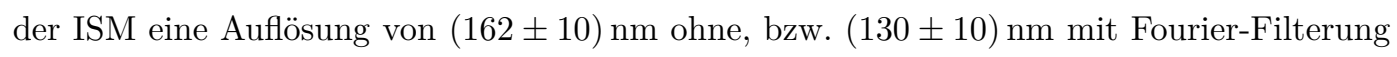
an $[76]^{(15)}$. Mit dem Re-scan Confocal Microscopy genannten Ansatz von De Luca et al. konnte ohne Nachbearbeitung eine Auflösung von $(170 \pm 10)$ nm erreicht werden [78] ${ }^{(16)}$. Wobei zu beachten ist, dass im hier vorgestellten Experiment nur 13 Detektoren verwendet wurden. Bei den Kamera-basierten ISM Methoden wird der Anregungspunkt mit deutlich mehr Detektorelementen aufgenommen. Prinzipiell sollte sich die im Experiment erreichte Auflösungsverbesserung durch den Einsatz weiterer Detektoren noch steigern lassen.

\subsubsection{Fluoreszenzlebensdauer-Mikroskopie}

Im Gegensatz den bisher vorgestellten Varianten der Image-Scanning-Mikroskopie, ermöglicht der MDS-Versuchsaufbau die Aufnahme von Fluoreszenz-Abklingkurven. Da das Zeit-

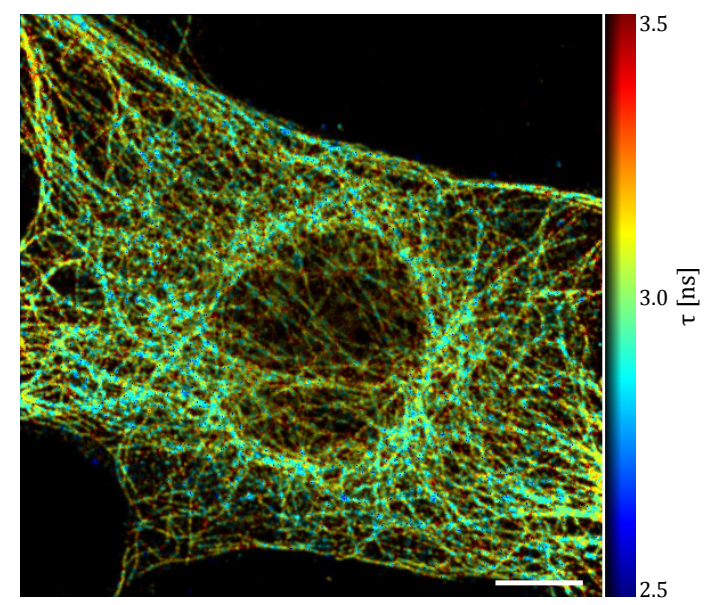

Abbildung 2.42: MDS-FLIM-Bild der
BODIPY FL markierten Mikrotubuli-Filamente
aus Abbildung 2.41. (Anregungsleistung $8 \mu \mathrm{W}$
bei $485 \mathrm{~nm}$, Maßstab: $5 \mu \mathrm{m})$

(15) Halbwertsbreite von Aktinfilamenten, markiert mit Alexa 488-phalloidin und angeregt bei $488 \mathrm{~nm}$.

${ }^{(16)}$ Halbwertsbreite von $100 \mathrm{~nm}$ Beads, angeregt bei $488 \mathrm{~nm}$. 


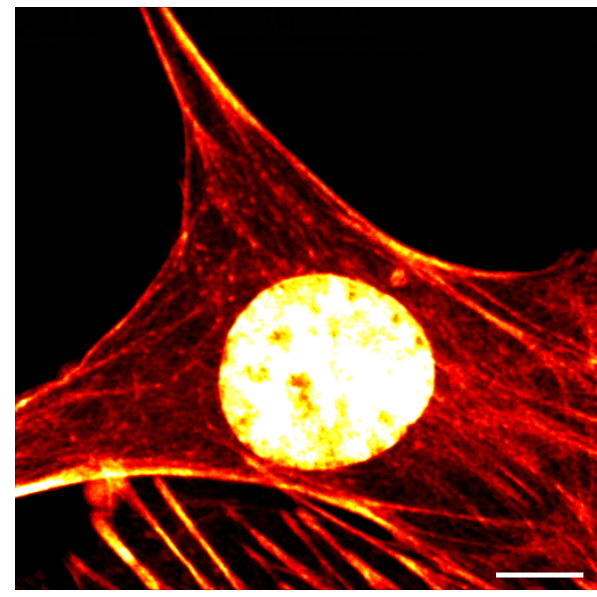

(a) MDS

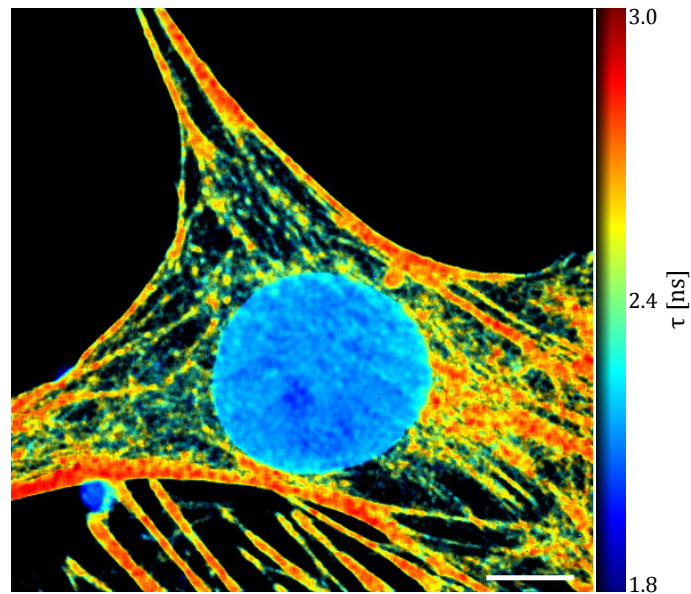

(b) MDS-FLIM

Abbildung 2.43: Aktinfilamente und Nukleus einer fixierten BPAE-Zelle markiert mit Texas Red-X Phalloidin und DAPI. (Anregungsleistung $14 \mathrm{~mW}$ bei $755 \mathrm{~nm}$ ) a: MDS-Bild. b: MDSFLIM-Bild mit farbkodierter Fluoreszenzlebensdauer. (Maßstab: $5 \mu \mathrm{m}$ )

signal der gepulsten Anregung erfasst wird, kann die Ankunftszeit jedes Photons relativ zu dem Laserpuls bestimmt werden. Somit lässt sich ein zeitaufgelöster MDS-Bildstapel erstellen und daraus mit einer geeigneten Software die räumliche Verteilung der Fluoreszenzlebensdauer visualisieren.

In Abbildung 2.42 ist eine entsprechende Auswertung für die Daten der Mikrotubuli-Filamente aus Abbildung 2.41 illustriert. In der Falschfarbendarstellung ist zu erkennen, dass die Fluoreszenzlebensdauer des BODYPI FL Farbstoff eine relativ homogene räumliche Verteilung aufweist. Im Mittel beträgt die Lebensdauer $\sim 3.1 \mathrm{~ns}$. Die laterale Auflösung der Daten entspricht der des MDS-Intensitätsbildes in Abbildung 2.41b.

Die Abbildung 2.43 zeigt Aktinfilamente sowie Nukleus einer BPAE-Zelle, die mit Texas RedX Phalloidin bzw. DAPI markiert wurden. Durch Zwei-Photonen-Absorption bei $755 \mathrm{~nm}$ konnten beide Farbstoffe gleichzeitig angeregt werden [6]. Der DNA-bindende Farbstoff DAPI hat bei dieser Wellenlänge einen höheren Zwei-Photonen-Wirkungsquerschnitt als Texas Red, wodurch der Zellkern in Abbildung 2.43a heller als die Aktinfilamente erscheint [114]. In dem FLIM-Bild auf der rechten Seite sind die unterschiedlichen Fluoreszenzlebensdauern der beiden Farbstoffe deutlich zu erkennen. Bei den Aktinfilamenten reichen die Werte von $2.5 \mathrm{~ns}$ bis $2.9 \mathrm{~ns}$. Die mittlere Lebensdauer im Nukleus beträgt hingegen $(2.12 \pm 0.04) \mathrm{ns}$. Damit ergibt sich eine gute Übereinstimmung mit dem Literaturwert von $2.07 \mathrm{~ns}$ für DNAgebundenes DAPI [115]. 
Ein limitierender Faktor von Einzelphotonendetektoren ist deren sog. Totzeit. Nach der Detektion eines Photons kann der Detektor für eine gewisse Zeit keine weiteren Photonen registrieren. Beim Überschreiten einer kritischen Zählrate kommt es dadurch zu einem sog. "pile-up", der einen nichtlinearen Zusammenhang zwischen gemessener und tatsächlicher Intensität zur Folge hat [99]. Bei FLIM kann das zu einer Verzerrung der Abklingkurve führen. Aus diesem Grund muss die Zählrate innerhalb des linearen Bereichs gehalten werden, wobei der maximale Wert je nach Detektortyp zwischen $500 \mathrm{kHz}$ und $10 \mathrm{MHz}$ betragen kann [116]. Die maximale Zählrate der hier verwendeten SPAD-Detektoren beträgt 1 MHz. Im Versuchsaufbau erlaubt die Verteilung der Intensität auf mehrere Detektoren allerdings deutlich höhere Zählraten. Über die zentrale Faser wird nur etwa ein sechstel der Gesamtintensität detektiert (siehe Abbildung 2.39). Dementsprechend kann, im Vergleich zu einem klassischen Detektionsschema mit einem einzelnen Detektor, eine sechsmal höher Zählrate erfasst werden. Bei einer ausreichend hellen Probe lässt sich die Aufnahmedauer somit signifikant reduzieren.

Chromatische Aberrationen haben sich bei den Versuchen mit Zwei-Photonen-Anregung als problematisch erwiesen. Die Scan- und Tubuslinse des Versuchsaufbaus sind nur in einem begrenzten Wellenlängenbereich auf chromatische Aberrationen korrigiert. Da das Anregungsund Emissionlicht beim beam-scanning unter variablem Winkel auf diese Linsen treffen, kommt es durch den großen Wellenlängenunterschied zu lateralen chromatischen Aberrationen. Das führt wiederum zu einer Translation der Intensiätsverteilung auf dem Faserbündel, die von der Auslenkung der Scanner abhängig ist. Bei Anregung im nahen Infrarot und Detektion um $500 \mathrm{~nm}$ betrug die Verschiebung vom Zentrum bis zum Rand des Bildes $\sim 30 \mu \mathrm{m}$ in jede Richtung. Diese Abweichung führt, besonders im äußeren Bereich, zu Ungenauigkeiten bei der Registrierung des MDS-Bildes. Aus diesem Grund war die Auflösungsverbesserung bei Zwei-Photonen-Anregung nur minimal. Durch Optimierung des optischen Systems sollte es in Zukunft möglich sein diesen Effekt zu reduzieren, sodass auch bei Zwei-PhotonenAnregung eine verbesserte Auflösung erreicht werden kann [117]. Alternativ könnten laterale chromatische Aberrationen durch eine Umstellung auf stage-scanning grundsätzlich vermieden werden, da der Strahlengang hierbei fix bleibt. 


\subsection{Fazit und Ausblick}

In diesem Teil der Arbeit wurde das Konzept der Image-Scanning-Mikroskopie mit fasergekoppelten Einzelphotonendetektoren umgesetzt. Mit dem vorgestellten Versuchsaufbau ist erstmals möglich die Aufösungsverbesserung durch ISM auf Fluoreszenzlebensdauer-Mikroskopie zu übertragen.

Im Vergleich zu etablierten Superresolution-Mikroskopie-Methoden ist die, dabei erreichte Auflösung von $(168 \pm 3) \mathrm{nm}$ (146 nm mit Dekonvolution) gering. Mit Methoden wie STED [52, 54, 118], PALM [119, 120] oder STORM [121] können Auflösungen weit unterhalb von $100 \mathrm{~nm}$ erreicht werden. Allerdings ist der Gewinn an Auflösung dabei oft mit anderen Nachteilen verbunden. Bei der STED-Mikroskopie kommt es zu verstärktem Fotobleichen der Proben und einer Verringerung der Signalintensität [122]. Außerdem ist der optische Aufbau komplexer und es wird ein zweiter Laser benötigt. Die Anwendung von PALM und STORM ist hingegen auf spezielle Farbstoffe beschränkt. Bei der hier vorgestellte Methode gibt es keinen inhärenten Nachteil. Detektionseffizienz und Aufnahmegeschwindigkeit sind prinzipiell identisch mit denen eines gewöhnlichen CLSM und auch der optische Aufbau unterscheidet sich kaum.

Ein zusätzlicher Vorteil von MDS besteht in der Verteilung der Signalintensität auf mehrere Detektoren. Dadurch sind deutlich höhere Zählraten möglich, als für TCSPC-Methoden mit nur einem Detektor. Bei Proben mit hoher Farbstoffdichte können so neben einer besseren lateralen Auflösung und Detektionseffizienz auch höhere Aufnahmegeschwindigkeiten erreicht werden.

Die gemessene Punktspreizfunktion deutet darauf hin, dass die Aufösung im Experiment vor allem durch die aufgetretenen Aberrationen limitiert wird. Die maximale Auflösungsverbesserung lässt sich nur erreichen, wenn sowohl die Intensitätsverteilung des Anregungslichts als auch die PSF der Detektion beugungsbegrenzt sind. Folglich sollte eine Optimierung des Versuchsaufbaus im Hinblick auf Aberrationen, die erreichbare Auflösungen erhöhen. Bei Anwendungen, die keine hohe Anforderung an die Aufnahmegeschwindigkeit stellen, ist es eventuell von Vorteil stage-scanning anstelle von beam-scanning zu verwenden um Abbildungsfehler zu minimieren. Die Erweiterung des Versuchsaufbaus um eine zweite Messkarte sowie zusätzliche Detektoren ermöglicht die Nutzung aller 19 Fasern des Bündels, wodurch sich Detektionseffizienz sowie Auflösung noch weiter steigern ließen. 


\section{A Anhang}

\section{A.1 Datenerfassungssoftware (Auszug)}

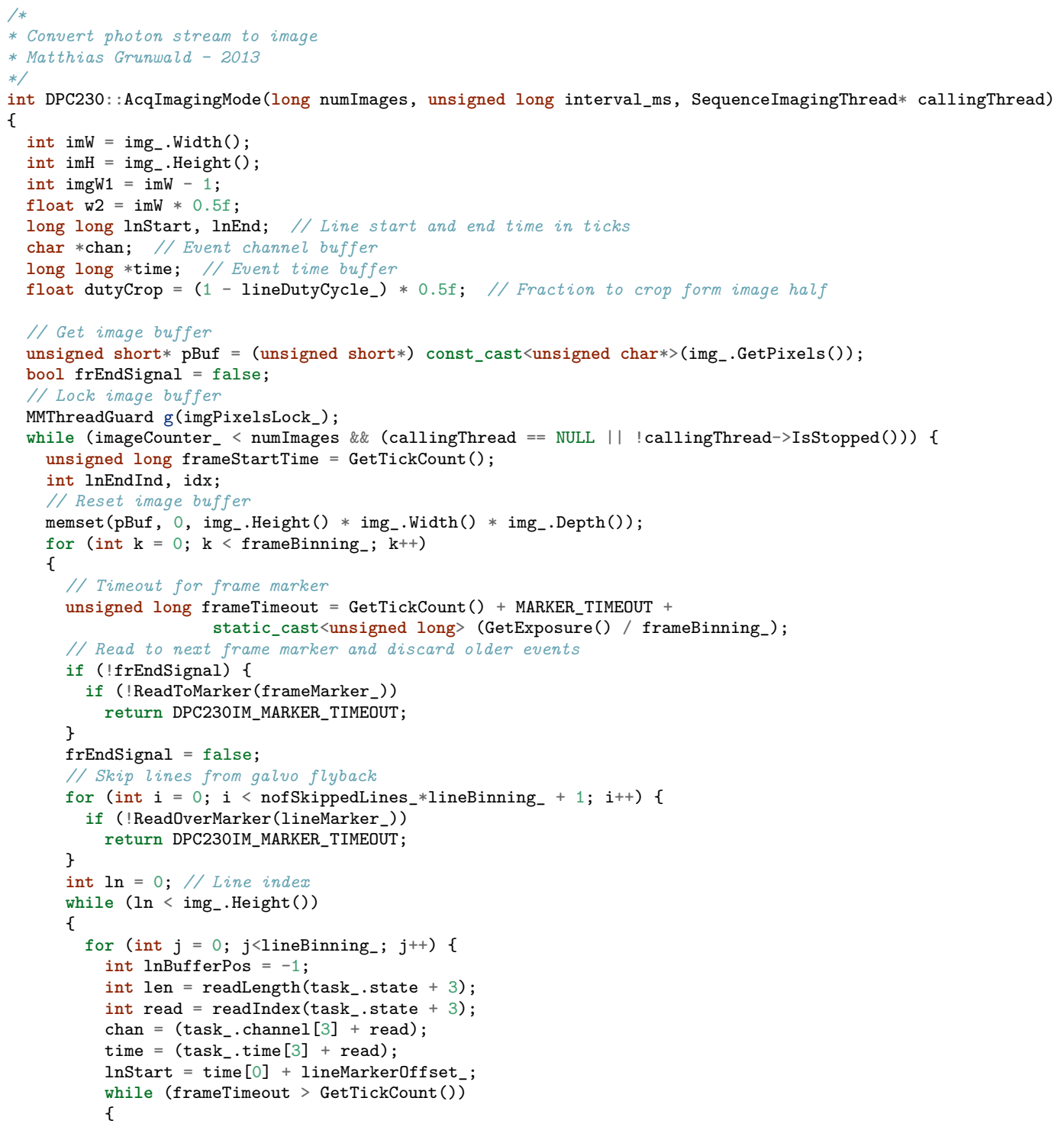




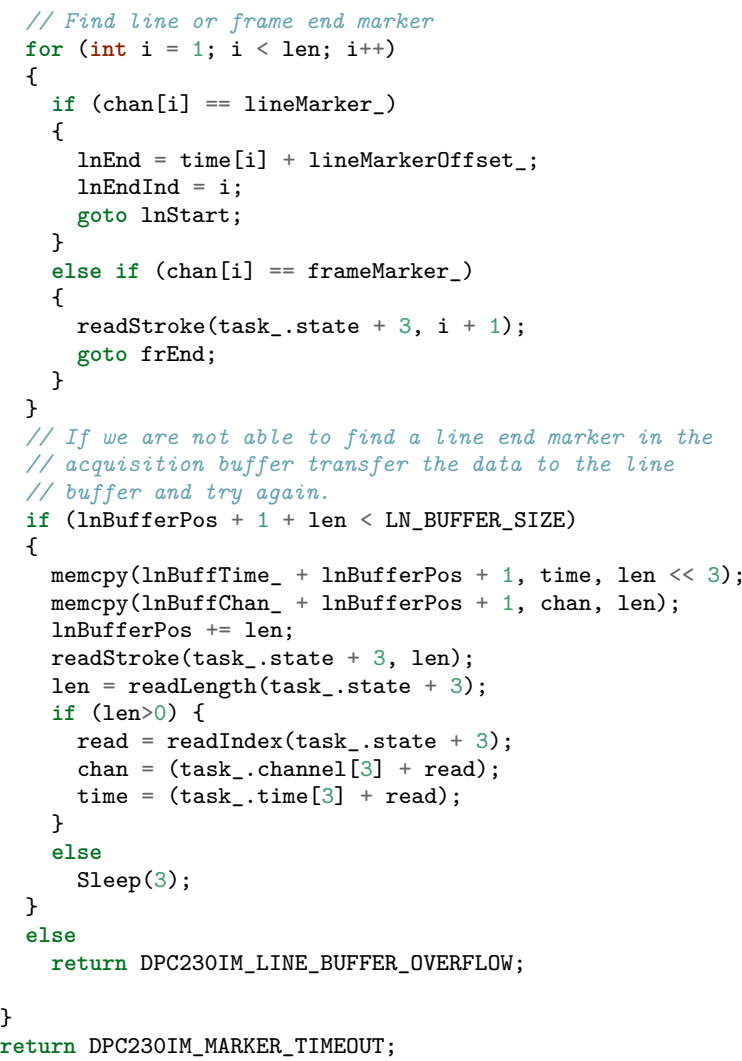




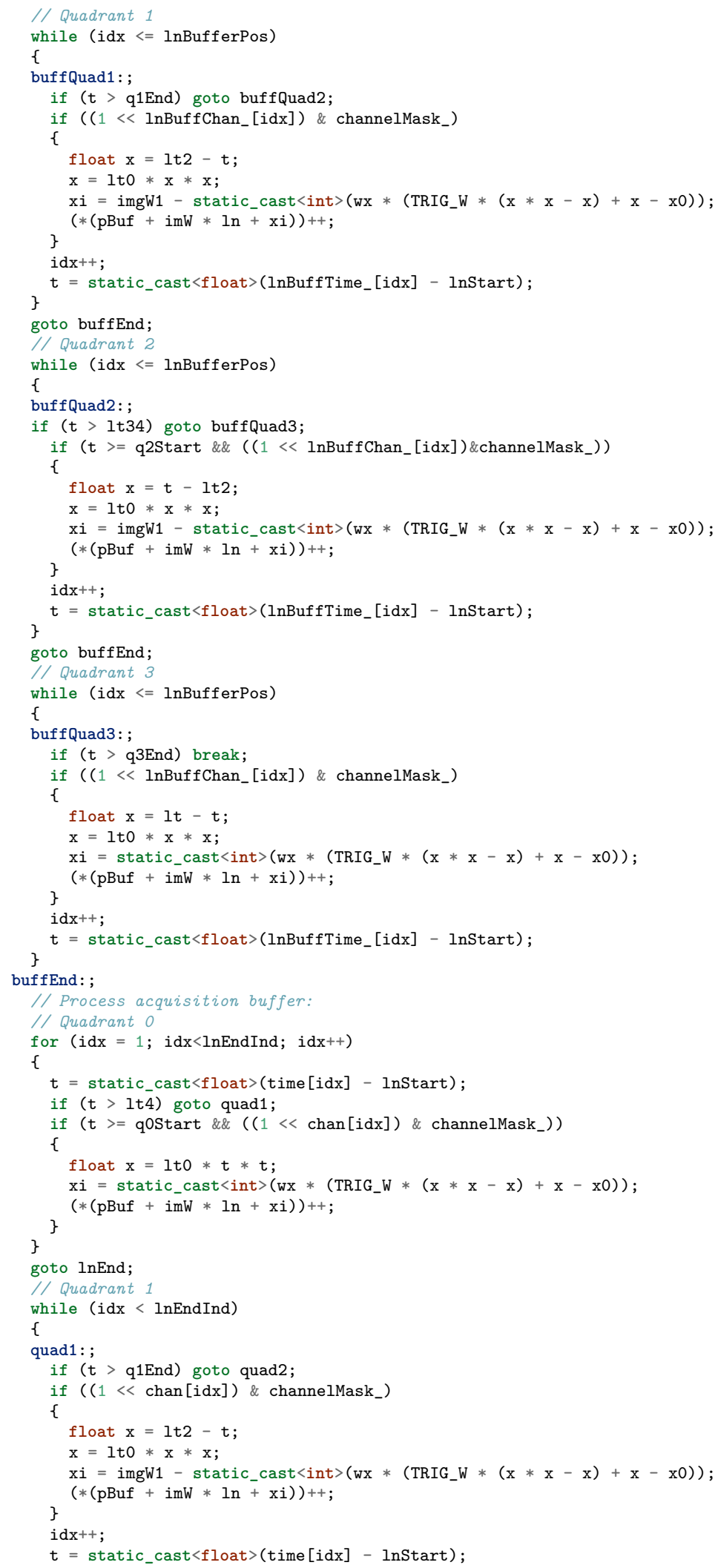




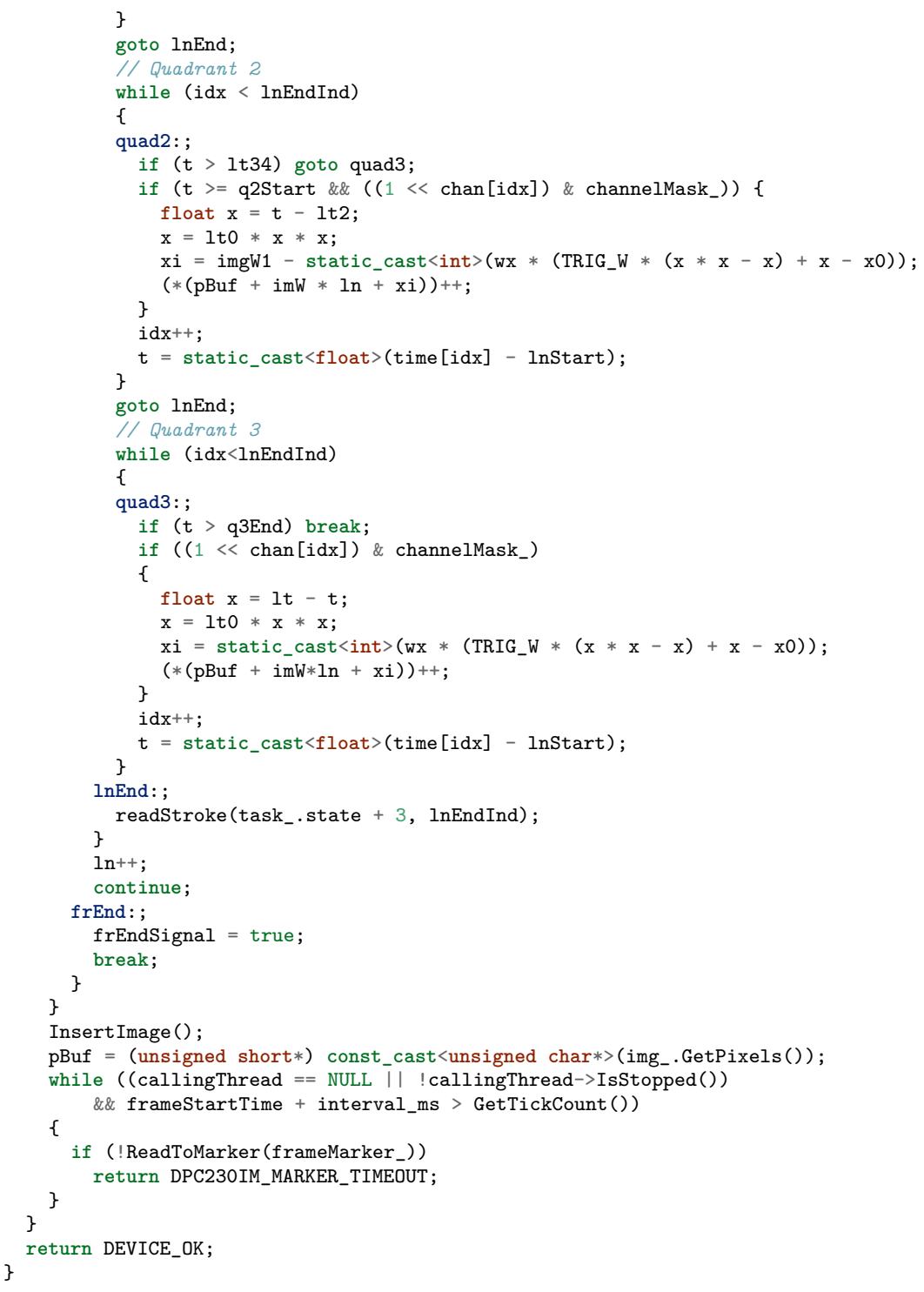




\section{A.2 Registrierung von MDS-Daten}

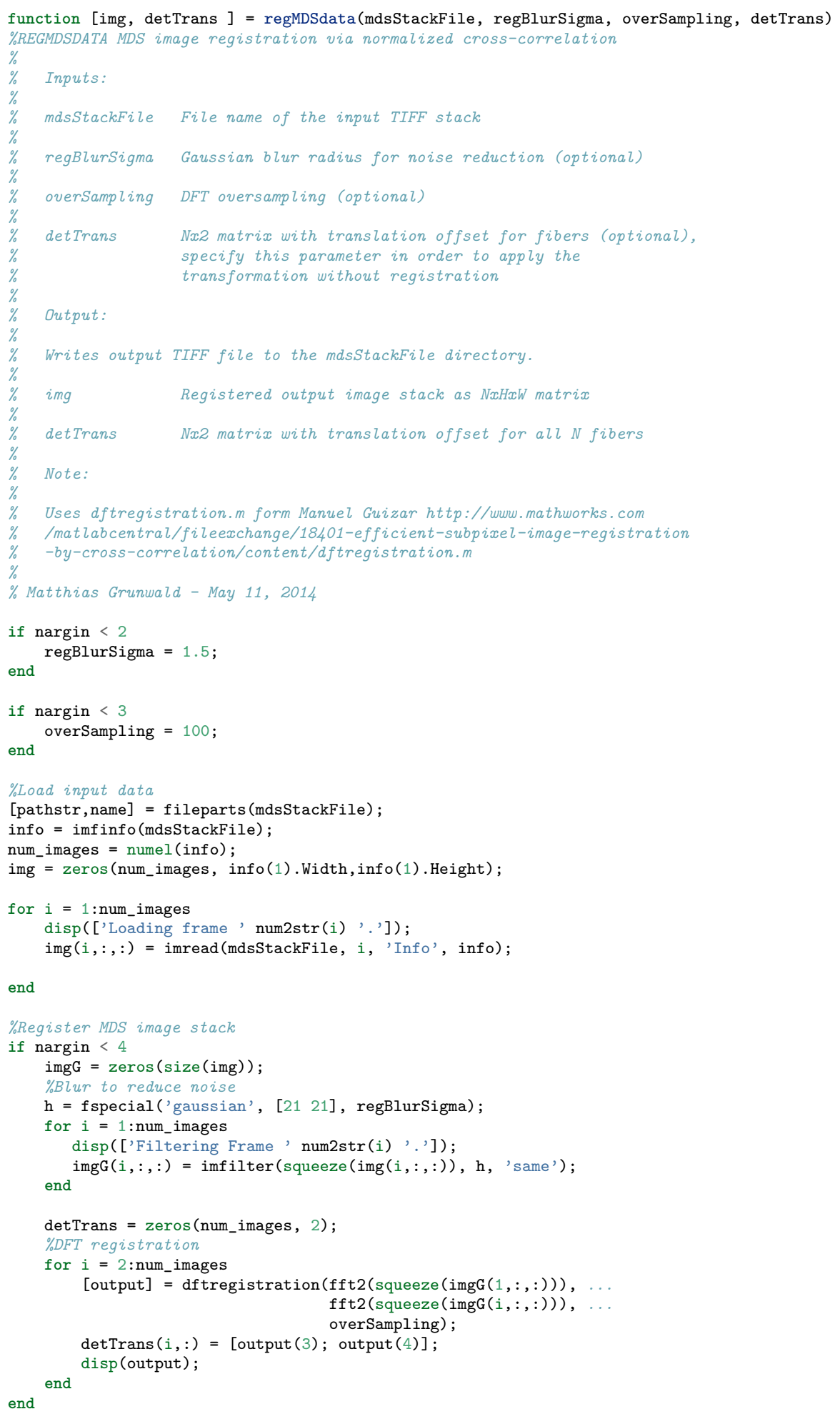




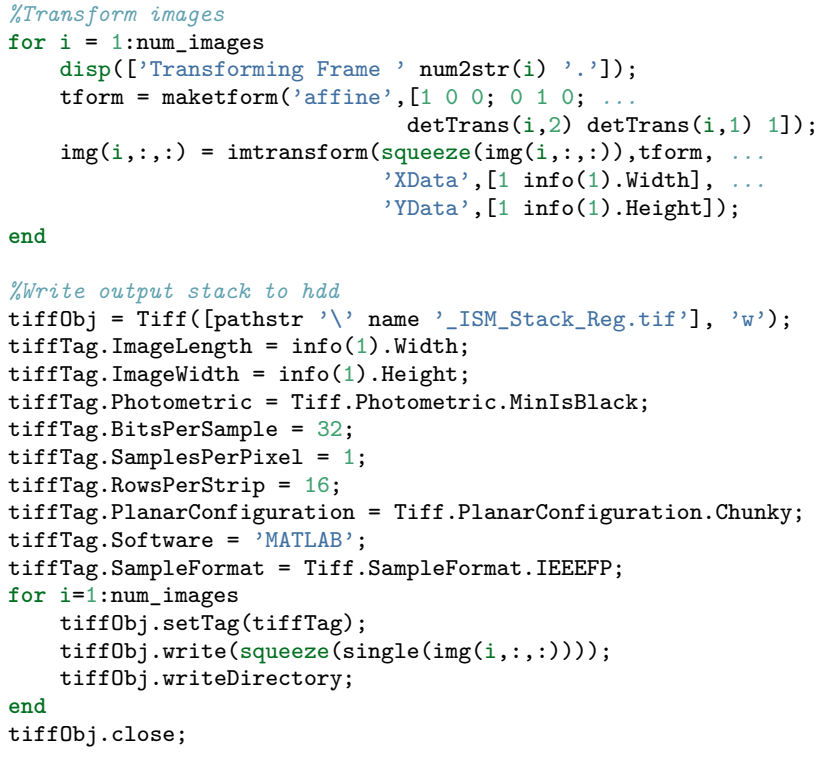

end 


\section{A.3 PSF Extraktion}

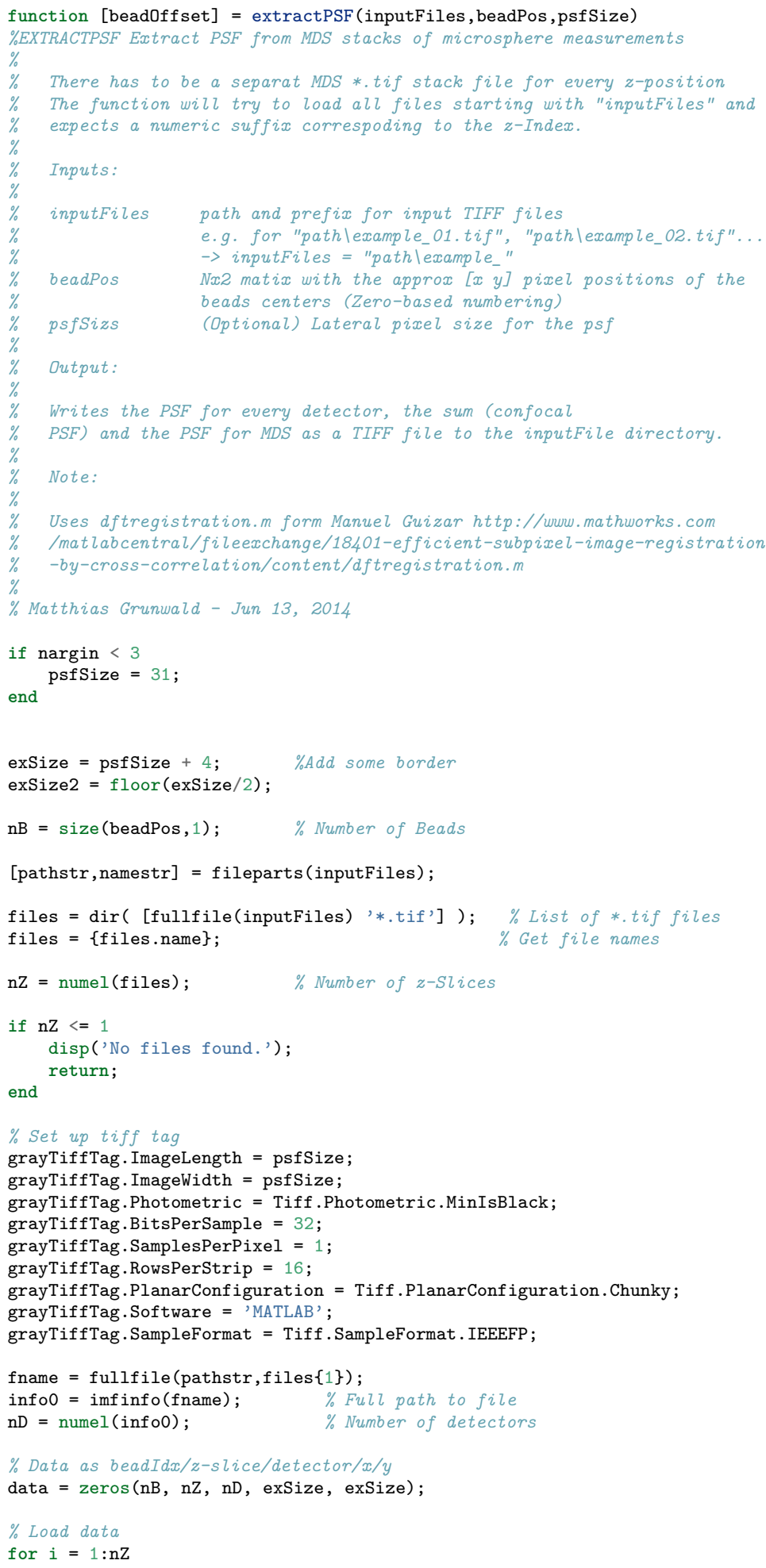




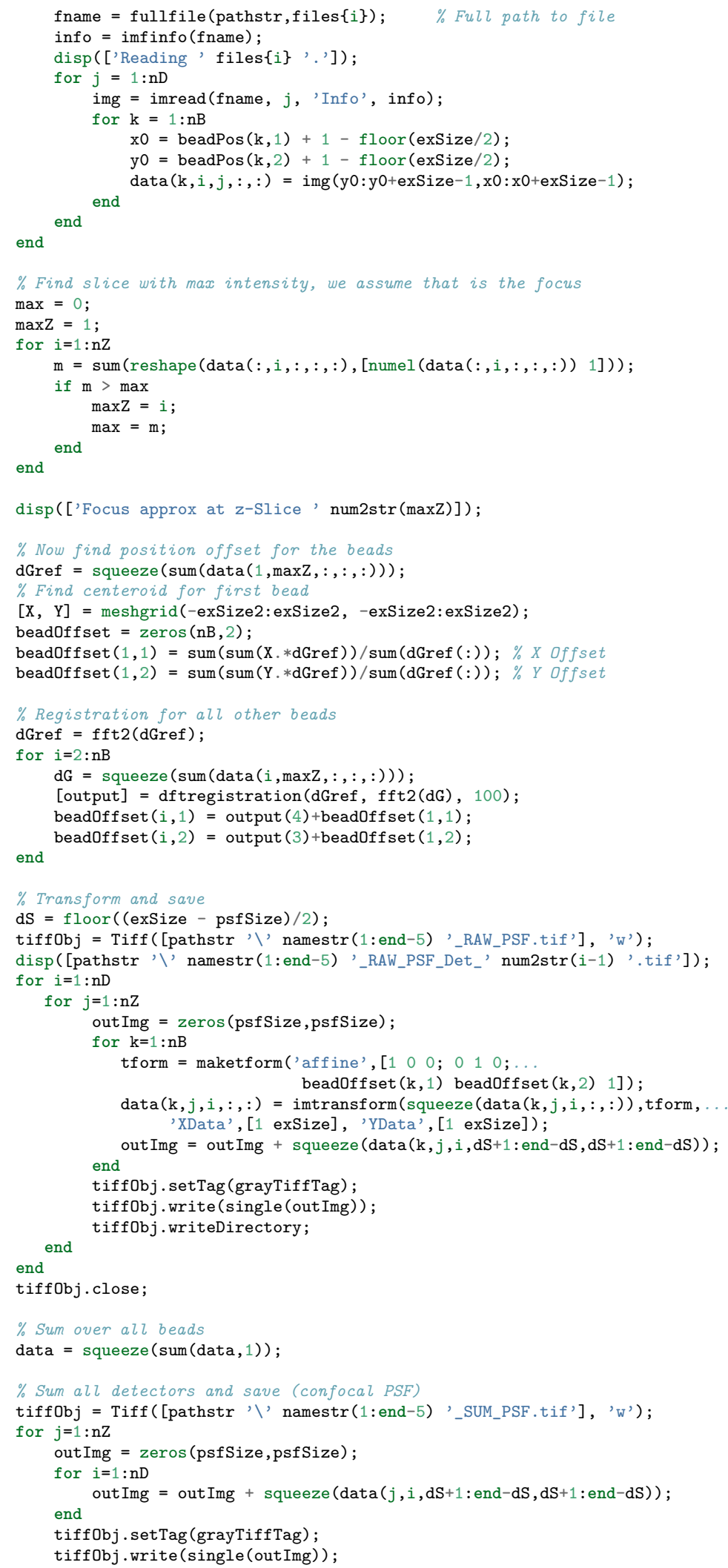


tiffobj.close

$\%$ Register PSFS of fibers
ismOffset $=\operatorname{zeros}(\mathrm{nD}, 2) ;$
$\operatorname{dGref}=\operatorname{fft} 2(\operatorname{squeeze}(\operatorname{data}(\operatorname{maxZ}, 1,:,:))) ;$

for $i=2: n B$

$\mathrm{dG}=$ squeeze $(\operatorname{data}(\max Z, i,:,:)) ;$

[output $]=$ dftregistration $(\mathrm{dGref}, \mathrm{fft} 2(\mathrm{dG}), 100)$;

$i \operatorname{smOffset}(i, 1)=\operatorname{output}(4)$;

end $i \operatorname{smOff} \operatorname{set}(i, 2)=\operatorname{output}(3)$;

\% Translate and sum all detectors (MDS PSF)

tiffObj $=\operatorname{Tiff}([$ pathstr ' ' $\operatorname{namestr}(1:$ end-5) '_ISM_PSF.tif'], 'W');

for $j=1: n z$

outImg $=$ zeros $($ psfSize,psfSize $)$

for $i=1: \mathrm{nD}$

tform = maketform ('affine', $\left[\begin{array}{llllll}1 & 0 & 0 ; & 0 & 1 & 0\end{array} ;\right.$.

$i \operatorname{smOffset}(i, 1) i \operatorname{smOff\operatorname {set}}(i, 2) 1])$;

$\operatorname{data}(j, i,:,:)=$ imtransform $(\operatorname{squeeze}(\operatorname{data}(j, i,:,:)), \operatorname{tform}, \ldots$

'XData', [1 exSize], 'YData', [1 exSize]).

end

outImg $=$ outImg $+\operatorname{squeeze}(\operatorname{data}(j, i, \mathrm{dS}+1:$ end $-\mathrm{dS}, \mathrm{dS}+1:$ end $-\mathrm{dS}))$;

tiffObj.setTag (grayTiffTag);

tiffObj.write(single (outImg))

tiffObj.writeDirectory;

end

tiffobj.close;

end 


\section{A.4 Dekonvolution}

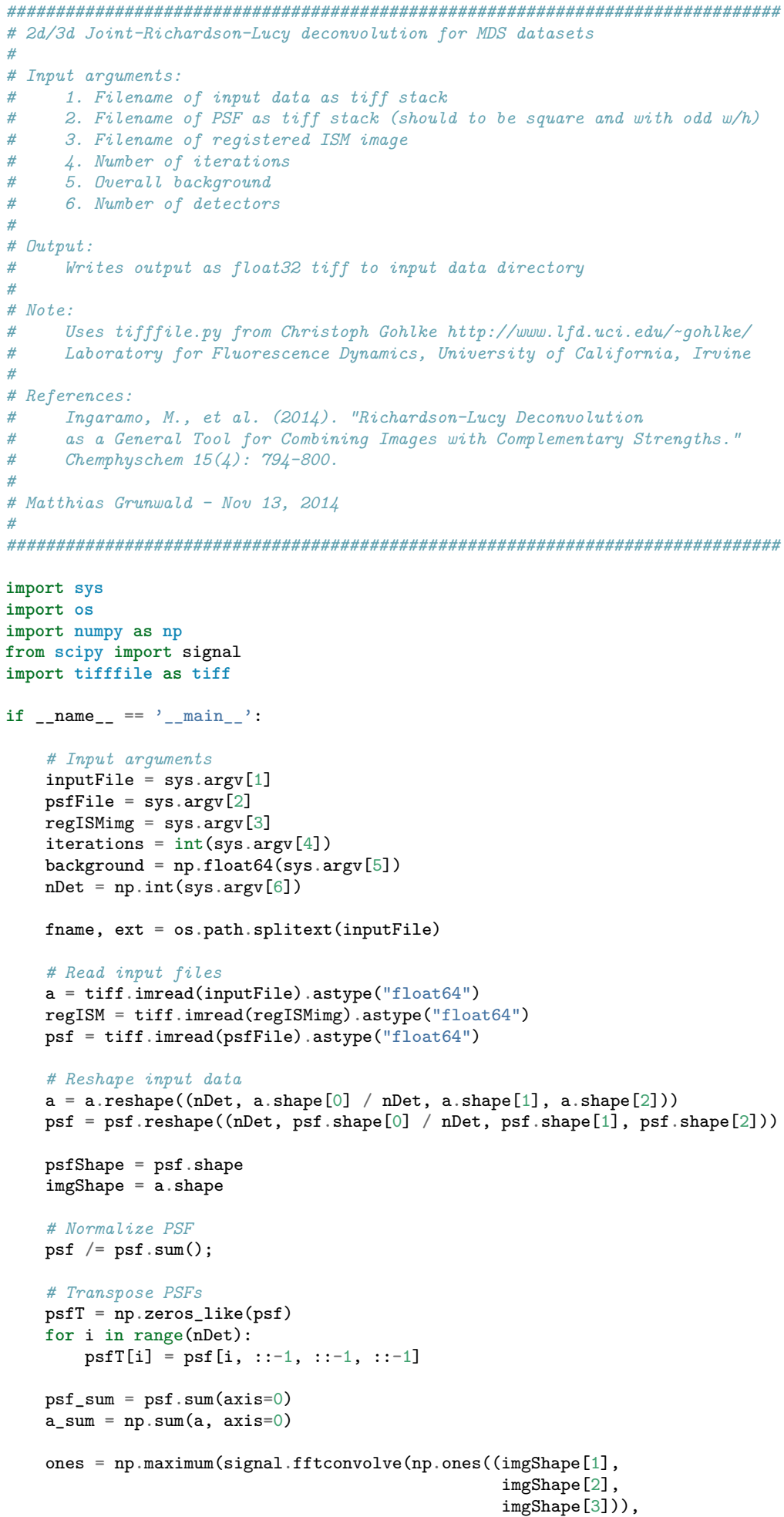




\section{psf_sum, 'full'), 0.01)}

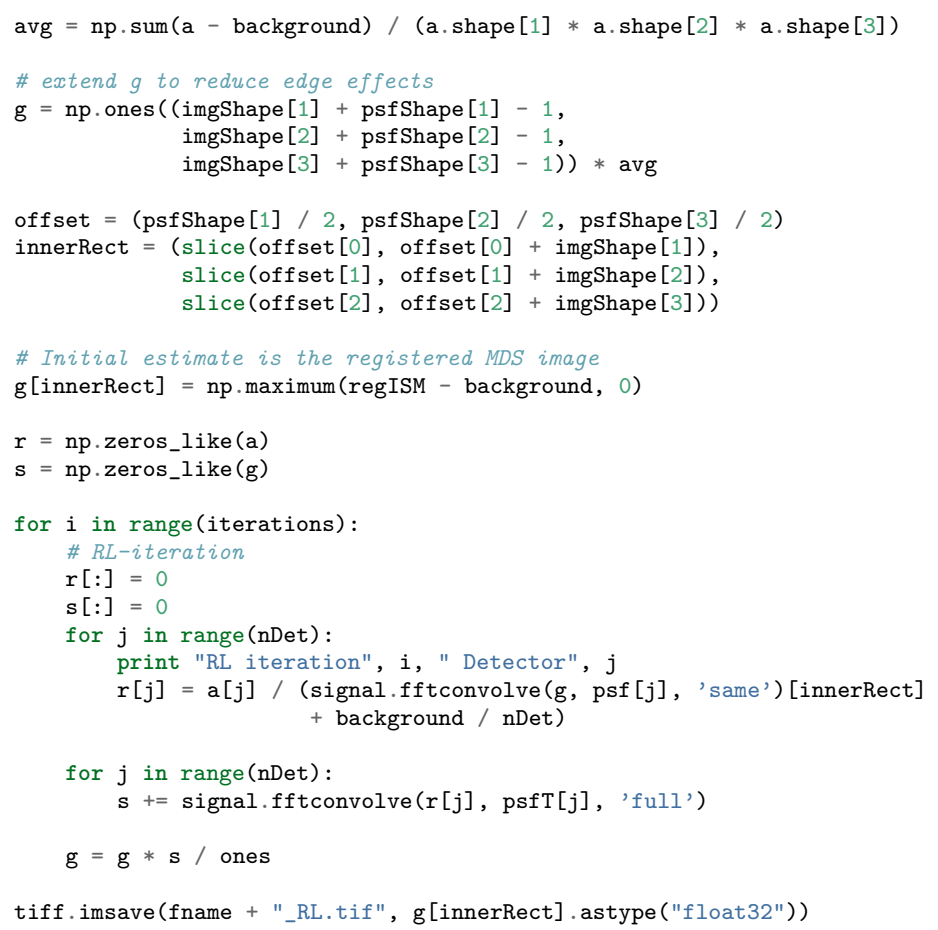

tiff.imsave(fname + "_RL.tif", g[innerRect].astype("float32")) 



\section{Literaturverzeichnis}

[1] B. R. Masters in eLS, John Wiley \& Sons, Ltd, 2001.

[2] F. C. Zanacchi, P. Bianchini, G. Vicidomini, Microscopy Research and Technique 2014, 77, 479-482.

[3] O. Heimstädt, Zs. f. wiss. Mikroskopie u. f. mikroskop. Technik 1911, 330-37.

[4] A. H. Coons, H. J. Creech, R. N. Jones, Experimental Biology and Medicine 1941, 47, 200-202.

[5] C. Lancaster, A focus on the history of light microscopy for cell culture, Bd. 6, 2014.

[6] M. Göppert-Mayer, Annalen der Physik 1931, 401, 273-294.

[7] W. Denk, J. Strickler, W. Webb, Science 1990, 248, 73-76.

[8] F. Helmchen, W. Denk, Nature Methods 2005, 2, 932-940.

[9] O. Shimomura, F. H. Johnson, Y. Saiga, Journal of Cellular and Comparative Physiology 1962, 59, 223-239.

[10] S. A. Rosenberg, M. E. Quinlan, J. N. Forkey, Y. E. Goldman, Accounts of Chemical Research 2005, 38, 583-593.

[11] M. P. Backlund, M. D. Lew, A. S. Backer, S. J. Sahl, W. E. Moerner, Chemphyschem 2014, 15, 587-599.

[12] K. Adachi, R. Yasuda, H. Noji, H. Itoh, Y. Harada, M. Yoshida, K. Kinosita, Proceedings of the National Academy of Sciences of the United States of America 2000, 97, 7243-7247.

[13] T. Nishizaka, K. Oiwa, H. Noji, S. Kimura, E. Muneyuki, M. Yoshida, K. Kinosita, Nature Structural \& Molecular Biology 2004, 11, 142-148.

[14] H. Sosa, E. J. G. Peterman, W. E. Moerner, L. S. B. Goldstein, Nature Structural 8 Molecular Biology 2001, 8, 540-544.

[15] A. B. Asenjo, N. Krohn, H. Sosa, Nature Structural \& Molecular Biology 2003, 10, $836-842$. 
[16] E. Betzig, R. J. Chichester, Science 1993, 262, 1422-1425.

[17] J. J. Macklin, J. K. Trautman, T. D. Harris, L. E. Brus, Science 1996, 272, 255-258.

[18] F. Güttler, M. Croci, A. Renn, U. P. Wild, Chemical Physics 1996, 211, 421-430.

[19] T. Ha, T. Enderle, D. S. Chemla, P. R. Selvin, S. Weiss, Physical Review Letters 1996, 77, 3979-3982.

[20] T. Ha, J. Glass, T. Enderle, D. S. Chemla, S. Weiss, Physical Review Letters 1998, 80, 2093-2096.

[21] T. Ha, T. A. Laurence, D. S. Chemla, S. Weiss, The Journal of Physical Chemistry $B$ 1999, 103, 6839-6850.

[22] K. D. Weston, L. S. Goldner, The Journal of Physical Chemistry B 2001, 105, 34533462.

[23] E. J. G. Peterman, H. Sosa, L. S. B. Goldstein, W. E. Moerner, Biophysical Journal 2001, 81, 2851-2863.

[24] J. T. Fourkas, Optics Letters 2001, 26, 211-213.

[25] J. N. Forkey, M. E. Quinlan, Y. E. Goldman, Biophysical Journal 2005, 89, 12611271.

[26] M. Böhmer, J. Enderlein, Journal of the Optical Society of America B 2003, 20, 554559 .

[27] D. Patra, I. Gregor, J. Enderlein, The Journal of Physical Chemistry A 2004, 108, 6836-6841.

[28] W. Schroeyers, R. Vallée, D. Patra, J. Hofkens, S. Habuchi, T. Vosch, M. Cotlet, K. Müllen, J. Enderlein, F. C. De Schryver, Journal of the American Chemical Society 2004, 126, 14310-14311.

[29] K. I. Mortensen, L. S. Churchman, J. A. Spudich, H. Flyvbjerg, Nature Methods 2010, 7, 377-381.

[30] F. Aguet, S. Geissbühler, I. Märki, T. Lasser, M. Unser, Optics Express 2009, 17, 6829-6848.

[31] A. S. Backer, M. P. Backlund, M. D. Lew, W. E. Moerner, Optics Letters 2013, 38, $1521-1523$.

[32] P. Atkins, R. Friedman, Molecular Quantum Mechanics, OUP Oxford, 2011.

[33] A. S. Coolidge, H. M. James, R. D. Present, The Journal of Chemical Physics 1936, 4, 193-211. 
[34] J. R. Lakowicz, Principles of fluorescence spectroscopy, 3rd, Springer, New York, 2006, xxvi, 954 p.

[35] B. R. Henry, M. Kasha, Annual Review of Physical Chemistry 1968, 19, 161-192.

[36] A. Einstein, Deutsche Physikalische Gesellschaft 1916, 18, 318-323.

[37] M. Born, E. Wolf, Principles of optics: electromagnetic theory of propagation, interference and diffraction of light, CUP Archive, 1999.

[38] B. Richards, E. Wolf, Proceedings of the Royal Society of London. Series A. Mathematical and Physical Sciences 1959, 253, 358-379.

[39] Rayleigh, Philosophical Magazine Series 5 1879, 8, 261-274.

[40] U. Kubitscheck, Fluorescence Microscopy: From Principles to Biological Applications, Wiley, 2013.

[41] E. Abbe, Archiv für mikroskopische Anatomie 1873, 9, 413-418.

[42] A. Matz, S. J. Lee, N. Schwedhelm-Domeyer, D. Zanini, A. Holubowska, M. Kannan, M. Farnworth, O. Jahn, M. C. Gopfert, J. Stegmuller, Cell Death and Differentiation 2015, 22, 626-642.

[43] P. Boccacci, Introduction to Inverse Problems in Imaging, Taylor \& Francis, 1998.

[44] P. Hansen, J. Nagy, D. O'Leary, Deblurring Images, Society for Industrial und Applied Mathematics, 2006, S. 129.

[45] L. I. Rudin, S. Osher, E. Fatemi, Physica D: Nonlinear Phenomena 1992, 60, 259268 .

[46] E. J. Candes, M. B. Wakin, Signal Processing Magazine IEEE 2008, 25, 21-30.

[47] B. K. Natarajan, SIAM J. Comput. 1995, 24, 227-234.

[48] E. J. Candès, J. K. Romberg, T. Tao, Communications on Pure and Applied Mathematics 2006, 59, 1207-1223.

[49] J. Harris, H. Stöcker, Handbook of Mathematics and Computational Science, Springer, 1998.

[50] A. Beck, M. Teboulle, SIAM Journal on Imaging Sciences 2009, 2, 183-202.

[51] M. Hirsch, R. J. Wareham, M. L. Martin-Fernandez, M. P. Hobson, D. J. Rolfe, PloS One 2013, 8, e53671.

[52] S. W. Hell, J. Wichmann, Optics Letters 1994, 19, 780-782.

[53] S. W. Hell, Nat Biotech 2003, 21, 1347-1355.

[54] S. W. Hell, Science 2007, 316, 1153-1158. 
[55] A. J. Cross, G. R. Fleming, Biophysical Journal 1984, 46, 45-56.

[56] I. Gryczynski, H. Malak, J. R. Lakowicz, Chemical Physics Letters 1995, 245, 30-35.

[57] E. Rusinova, V. Tretyachenko-Ladokhina, O. E. Vele, D. F. Senear, J. A. Ross, Analytical Biochemistry 2002, 308, 18-25.

[58] D. Wildanger, E. Rittweger, L. Kastrup, S. W. Hell, Optics Express 2008, 16, 96149621.

[59] A. Penzkofer, J. Wiedmann, Optics Communications 1980, 35, 81-86.

[60] F. López Arbeloa, V. Martínez Martínez, Chemistry of Materials 2006, 18, 14071416 .

[61] J.-i. Hotta, E. Fron, P. Dedecker, K. P. F. Janssen, C. Li, K. Müllen, B. Harke, J. Bückers, S. W. Hell, J. Hofkens, Journal of the American Chemical Society 2010, 132, 5021-5023.

[62] C. Eggeling, A. Volkmer, C. A. Seidel, Chemphyschem 2005, 6, 791-804.

[63] R. W. Cole, T. Jinadasa, C. M. Brown, Nat. Protocols 2011, 6, 1929-1941.

[64] P. Beckmann, Acta Technica 1959, 4, 323-335.

[65] C. M. Pieper, J. Enderlein, Chemical Physics Letters 2011, 516, 1-11.

[66] J. S. Hyams, C. W. Lloyd, Microtubules, Wiley-Liss, New York, 1994.

[67] I. Testa, C. A. Wurm, R. Medda, E. Rothermel, C. von Middendorf, J. Fölling, S. Jakobs, A. Schönle, S. W. Hell, C. Eggeling, Biophysical Journal 2010, 99, 26862694 .

[68] A. M. Rossi, C. W. Taylor, Nat. Protocols 2011, 6, 365-387.

[69] C. M. Yengo, C. L. Berger, Current Opinion in Pharmacology 2010, 10, 731-7.

[70] A. Gasecka, T. J. Han, C. Favard, B. R. Cho, S. Brasselet, Biophysical Journal 2009, 97, $2854-62$.

[71] P. Ferrand, P. Gasecka, A. Kress, X. Wang, F.-Z. Bioud, J. Duboisset, S. Brasselet, Biophysical Journal 2014, 106, 2330-2339.

[72] C. B. Müller, J. Enderlein, Physical Review Letters 2010, 104, 198101.

[73] C. J. R. Sheppard, Optik 1988, 80, 53-54.

[74] M. G. L. Gustafsson, Journal of Microscopy 2000, 198, 82-87.

[75] C. J. R. Sheppard, S. B. Mehta, R. Heintzmann, Optics Letters 2013, 38, 2889-2892.

[76] O. Schulz, C. Pieper, M. Clever, J. Pfaff, A. Ruhlandt, R. H. Kehlenbach, F. S. Wouters, J. Großhans, G. Bunt, J. Enderlein, Proceedings of the National Academy of Sciences 2013, 110, 21000-21005. 
[77] A. G. York, S. H. Parekh, D. D. Nogare, R. S. Fischer, K. Temprine, M. Mione, A. B. Chitnis, C. A. Combs, H. Shroff, Nature Methods 2012, 9, 749-754.

[78] G. M. R. De Luca, R. M. P. Breedijk, R. A. J. Brandt, C. H. C. Zeelenberg, B. E. de Jong, W. Timmermans, L. N. Azar, R. A. Hoebe, S. Stallinga, E. M. M. Manders, Biomedical Optics Express 2013, 4, 2644-2656.

[79] A. G. York, P. Chandris, D. D. Nogare, J. Head, P. Wawrzusin, R. S. Fischer, A. Chitnis, H. Shroff, Nature Methods 2013, 10, 1122-1126.

[80] A. Restelli, I. Rech, P. Maccagnani, M. Ghioni, S. Cova, Journal of Modern Optics 2007, 54, 213-223.

[81] M. Vitali, D. Bronzi, A. J. Krmpot, S. N. Nikolic, F. J. Schmitt, C. Junghans, S. Tisa, T. Friedrich, V. Vukojevic, L. Terenius, F. Zappa, R. Rigler, Selected Topics in Quantum Electronics IEEE Journal of 2014, 20, 344-353.

[82] A. Miyawaki, J. Llopis, R. Heim, J. M. McCaffery, J. A. Adams, M. Ikura, R. Y. Tsien, Nature 1997, 388, 882-7.

[83] A. Celli, S. Sanchez, M. Behne, T. Hazlett, E. Gratton, T. Mauro, Biophysical Journal 2010, 98, 911-921.

[84] R. Sanders, A. Draaijer, H. C. Gerritsen, P. M. Houpt, Y. K. Levine, Analytical Biochemistry 1995, 227, 302-308.

[85] C. Hille, M. Berg, L. Bressel, D. Munzke, P. Primus, H. G. Lohmannsroben, C. Dosche, Analytical and Bioanalytical Chemistry 2008, 391, 1871-9.

[86] M. Stöckl, A. P. Plazzo, T. Korte, A. Herrmann, Journal of Biological Chemistry 2008, 283, 30828-30837.

[87] A. Bunge, M. Fischlechner, M. Loew, A. Arbuzova, A. Herrmann, D. Huster, Soft Matter 2009, 5, 3331-3339.

[88] M. C. Skala, K. M. Riching, A. Gendron-Fitzpatrick, J. Eickhoff, K. W. Eliceiri, J. G. White, N. Ramanujam, Proceedings of the National Academy of Sciences 2007, 104, 19494-19499.

[89] H. Wallrabe, A. Periasamy, Current Opinion in Biotechnology 2005, 16, 19-27.

[90] M. Minsky, Scanning 1988, 10, 128-138.

[91] J. Pawley, Handbook of Biological Confocal Microscopy, Springer US, 2010.

[92] M. Gu, Principles of Three Dimensional Imaging in Confocal Microscopes, World Scientific, 1996.

[93] G. Cox, C. J. R. Sheppard, Microscopy Research and Technique 2004, 63, 18-22. 
[94] M. Ingaramo, A. G. York, P. Wawrzusin, O. Milberg, A. Hong, R. Weigert, H. Shroff, G. H. Patterson, Proceedings of the National Academy of Sciences 2014, 111, 52545259 .

[95] W. H. Richardson, Journal of the Optical Society of America 1972, 62, 55-59.

[96] L. Lucy, The astronomical journal 1974, 79, 745.

[97] M. Ingaramo, A. G. York, E. Hoogendoorn, M. Postma, H. Shroff, G. H. Patterson, Chemphyschem 2014, 15, 794-800.

[98] F. Ströhl, C. F. Kaminski, Methods and Applications in Fluorescence 2015, 3, 014002.

[99] W. Becker, Advanced Time-Correlated Single Photon Counting Techniques, Springer, Berlin, 2005.

[100] Q. T. Nguyen, N. Callamaras, C. Hsieh, I. Parker, Cell Calcium 2001, 30, 383-393.

[101] J. Bland-Hawthorn, J. Bryant, G. Robertson, P. Gillingham, J. O?Byrne, G. Cecil, R. Haynes, S. Croom, S. Ellis, M. Maack, P. Skovgaard, D. Noordegraaf, Optics Express 2011, 19, 2649-2661.

[102] A. Edelstein, N. Amodaj, K. Hoover, R. Vale, N. Stuurman in Current Protocols in Molecular Biology, John Wiley \& Sons, Inc., 2001.

[103] W. K. Pratt, New York 1978, 1191, 491-556.

[104] M. Guizar-Sicairos, S. T. Thurman, J. R. Fienup, Optics Letters 2008, 33, 156-158.

[105] E. O. Brigham, E. Brigham, The fast Fourier transform and its applications, Bd. 1, Prentice Hall Englewood Cliffs, NJ, 1988.

[106] T. E. Oliphant, Computing in Science \& Engineering 2007, 9, 10-20.

[107] S. C. Warren, A. Margineanu, D. Alibhai, D. J. Kelly, C. Talbot, Y. Alexandrov, I. Munro, M. Katan, C. Dunsby, P. M. W. French, PloS One 2013, 8, e70687.

[108] A. Egner, S. Hell in Handbook Of Biological Confocal Microscopy, (Hrsg.: J. B. Pawley), Springer US, 2006, Kap. 20, S. 404-413.

[109] H. E. Keller in Handbook Of Biological Confocal Microscopy, (Hrsg.: J. B. Pawley), Springer US, 2006, Kap. 7, S. 145-161.

[110] I. Gregor, J. Enderlein, Optics Letters 2005, 30, 2527-2529.

[111] R. M. Zucker, O. T. Price, Methods 1999, 18, 447-58.

[112] U. S. Ryan, D. R. Schultz, P. J. Del Vecchio, J. W. Ryan, Science 1980, 208, 748-9.

[113] U. S. Ryan, Environmental Health Perspectives 1984, 56, 103-114. 
[114] F. Bestvater, E. Spiess, G. Stobrawa, M. Hacker, T. Feurer, T. Porwol, U. BerchnerPfannschmidt, C. Wotzlaw, H. Acker, Journal of Microscopy (Oxford) 2002, 208, 108-15.

[115] M. L. Barcellona, E. Gratton, European Biophysics Journal 1990, 17, 315-323.

[116] H. C. Gerritsen, M. A. H. Asselbergs, A. V. Agronskaia, W. G. J. H. M. Van Sark, Journal of Microscopy 2002, 206, 218-224.

[117] A. Negrean, H. D. Mansvelder, Biomedical Optics Express 2014, 5, 1588-1609.

[118] T. A. Klar, S. Jakobs, M. Dyba, A. Egner, S. W. Hell, Proceedings of the National Academy of Sciences 2000, 97, 8206-8210.

[119] E. Betzig, G. H. Patterson, R. Sougrat, O. W. Lindwasser, S. Olenych, J. S. Bonifacino, M. W. Davidson, J. Lippincott-Schwartz, H. F. Hess, Science 2006, 313, $1642-$ 1645 .

[120] S. T. Hess, T. P. K. Girirajan, M. D. Mason, Biophysical Journal 2006, 91, 42584272 .

[121] M. J. Rust, M. Bates, X. Zhuang, Nature Methods 2006, 3, 793-796.

[122] M. Dyba, S. W. Hell, Applied Optics 2003, 42, 5123-5129. 



\title{
Lebenslauf
}

\section{Persönliche Daten}

\author{
Matthias Grunwald \\ Planckstraße 10 \\ 37073 Göttingen \\ E-Mail: ma.grunwald@gmx.net \\ Geb. am 02. 01.1986 in Ostercappeln
}

\section{Schulbildung}

2002-2005

Söderblom-Gymnasium Espelkamp

\section{Studium}

10/2005-01/2011 Diplomstudium der Chemie an der Georg-August-Universität Göttingen 11/2007 Vordiplom

05/2009-10/2009 Praktikum bei der Firma Cognis in Düsseldorf

01/2011 Diplom

seit $2011 \quad$ Promotion am Max-Planck-Institut für biophysikalische Chemie

\section{Publikationen}

[1] W. Vennekate, S. Schroder, C. C. Lin, G. van den Bogaart, M. Grunwald, R. Jahn, P. J. Walla, PNAS 2012, 109, 11037-11042.

[2] L. Wilk, M. Grunwald, P.-N. Liao, P. J. Walla, W. Kühlbrandt, Direct interaction of the major light-harvesting complex II and PsbS in nonphotochemical quenching. PNAS 2013, 110, 5452-5456.

[3] N. Hafi, M. Grunwald, L. S. van den Heuvel, T. Aspelmeier, J.-H. Chen, M. Zagrebelsky, O. M. Schutte, C. Steinem, M. Korte, A. Munk, P. J. Walla, Fluorescence nanoscopy by polarization modulation and polarization angle narrowing. Nature Methods 2014, $11,579-584$.

[4] J. Chen, Y. Kellner, M. Zagrebelsky, M. Grunwald, M. Korte, P. J. Walla, Two-photon correlation spectroscopy in single dendritic spines reveals fast actin filament reorganization during activity-dependent growth, PLoS ONE 2015, 10(5): e0128241. 